\title{
Goals and status of the CBM experiment
}

Peter Senger

GSI Darmstadt, Germany

\begin{abstract}
The goal of the heavy-ion collision program at the future Facility for Antiproton and Ion Research (FAIR) in Darmstadt (Germany) is to explore the phase diagram of strongly interacting matter in the region of the highest baryon densities. The CBM research program addresses fundamental aspects of QCD such as the equation-of-state of highly compressed baryonic matter, the deconfinement phase transition and its critical endpoint, chiral symmetry restoration at high baryon densities, and the in-medium properties of hadrons. The corresponding key observables comprise low-mass vector mesons decaying into lepton pairs which serve as penetrating probes, hidden and open charm produced at threshold beam energies, (multi-) strange particles, and global features like collective flow and event-by-event fluctuations. The experimental task is to identify both hadrons and leptons and to detect rare probes in a heavy-ion environment. The experimental challenge is to select rare events in nucleus-nucleus collisions with charged particle multiplicities of about 1000 per central event at reaction rates of up to $10 \mathrm{MHz}$. Such measurements require fast and radiation hard detectors, fast and selftriggered read-out electronics, a high-speed data acquisition system, and online event selection based on full track reconstruction. The layout of the CBM detector system and the results of feasibility studies will be presented. More than 400 scientists from 50 institutions and 15 countries actually participate in the realization of the CBM experiment which represents a major international effort in future heavy-ion physics.
\end{abstract}

The participation at this summer school was partially supported by the HISS Dubna program of the Helmholtz association.

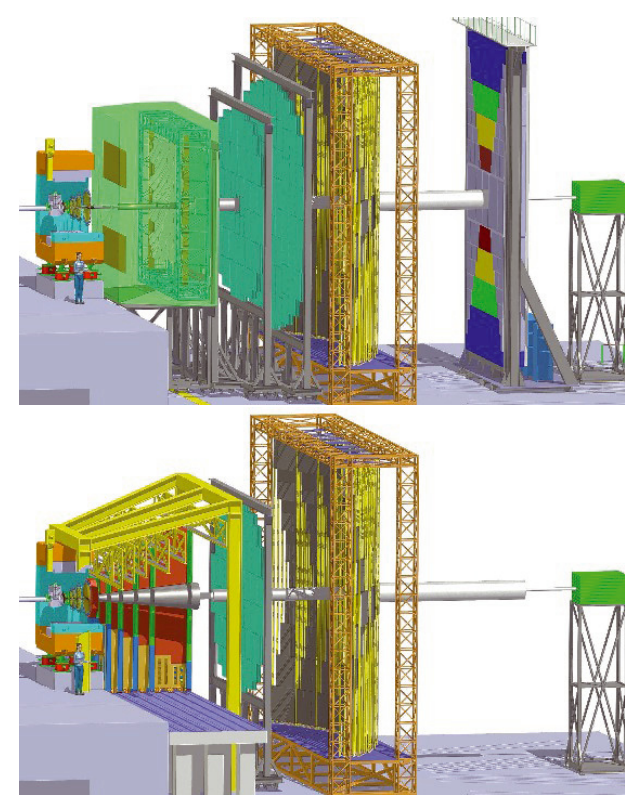

Fig. 1. The CBM experimental facility with electron detectors RICH and TRD (top panel) and with the muon detection system (bottom panel).

This is an Open Access article distributed under the terms of the Creative Commons Attribution-Noncommercial License 3.0, which permits unrestricted use, distribution, and reproduction in any noncommercial medium, provided the original work is properly cited 


\title{
Experiments with dense nuclear matter
}

\author{
Peter Senger (GSI)
}

\section{Outline:}

Part I: Dense baryonic matter in the laboratory:

$>$ Probing the nuclear equation-of-state by collective proton flow and strangeness production

$>$ Measuring in medium properties of strange mesons

Part II: Towards highest baryon densities :

$>$ Exploring the phases of QCD matter

HISS Dense Matter in HIC and Astrophysics, 14. - 26. 07. 2008, Dubna, Russia

"The challenge for the next century physics is:

explain confinement and broken (chiral) symmetry"

T.D. Lee

"But perhaps the most interesting and surprising thing about

QCD at high density is that, by thinking about it, one discovers

a fruitful new perspective on the traditional problem

of confinement and chiral-symmetry breaking".

F. Wilczek 


\section{Fundamental Questions of QCD}

$>$ What is the equation-of-state of strongly interacting matter? (core collapse supernovae, neutron stars, early universe)

$>$ What is the structure of strongly interacting matter as a function of $T$ and $\rho_{B}$ ? (hot and dense hadronic medium, deconfined phase, phase transitions?)

$>$ What are the in-medium properties of hadrons as a function of $T$ and $\rho_{B}$ ? (partial restoration of chiral symmetry?)

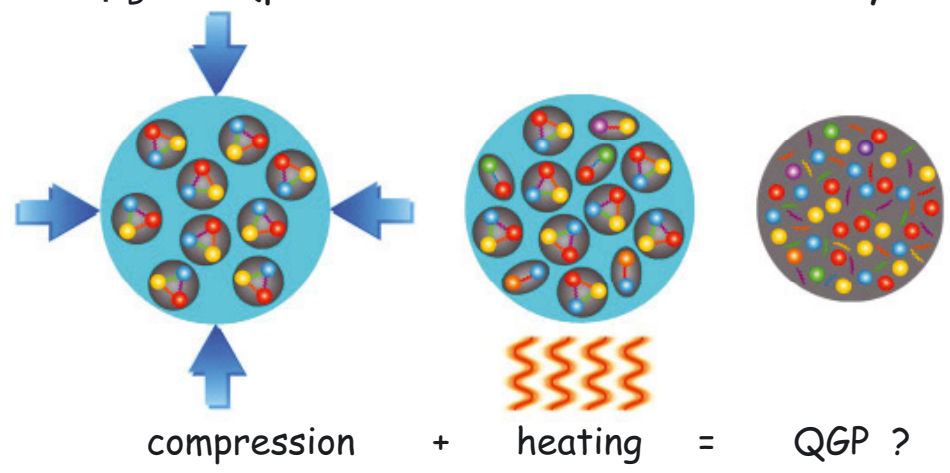

The phase diagram of strongly interacting matter

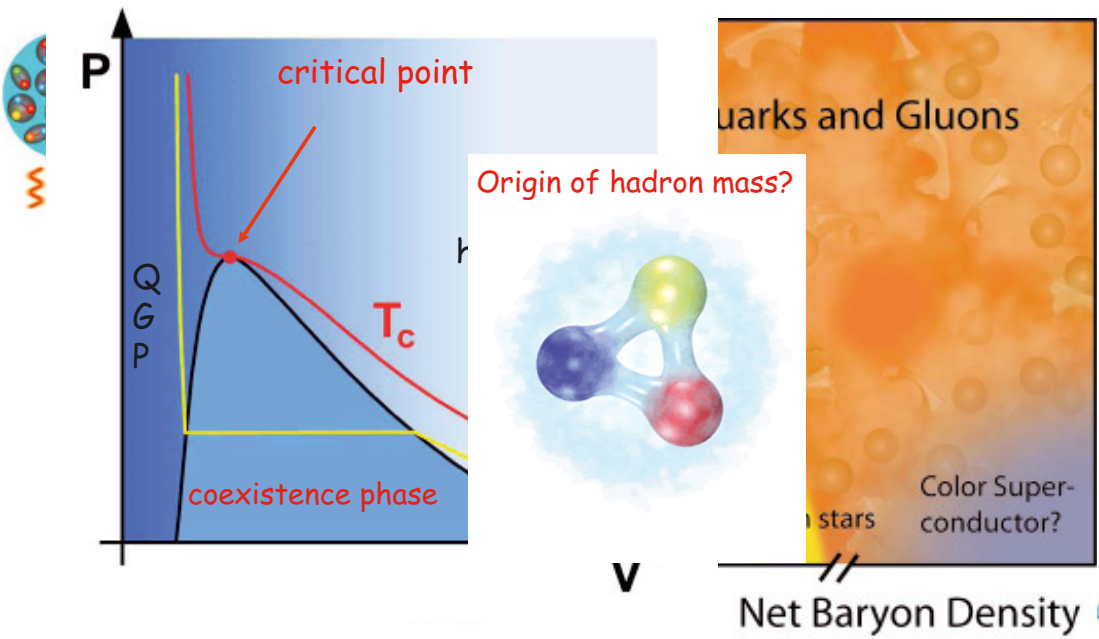

Are heavy ion collisions the appropriate tool to study QCD matter?

S Small, shortlived, dynamical systems: is equilibrated matter established?

$>$ Particles freeze out at $\rho<\rho_{0}$ : information on high density phase?

$>$ Do signatures from the partonic phase survive hadronization? 


\section{The evolution of matter in the universe}

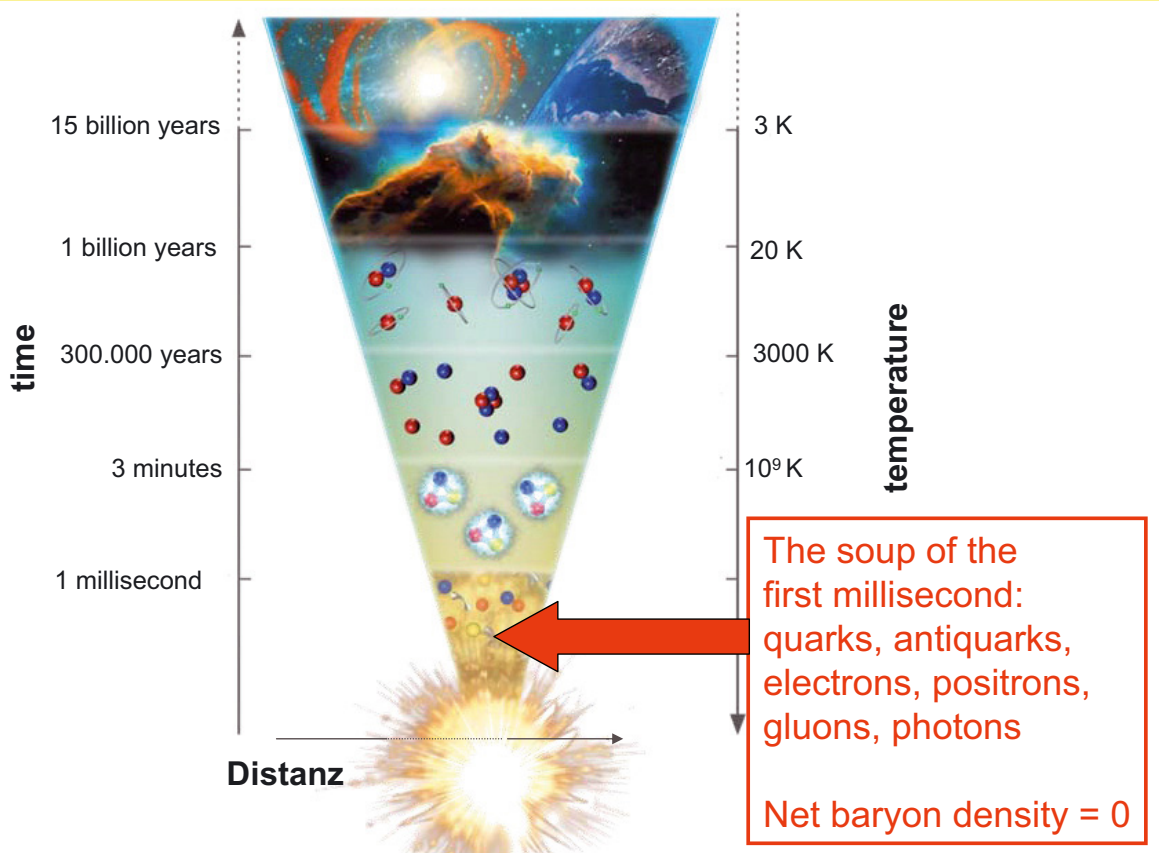

Dense nuclear matter in nature: Core collapse supernova explosion
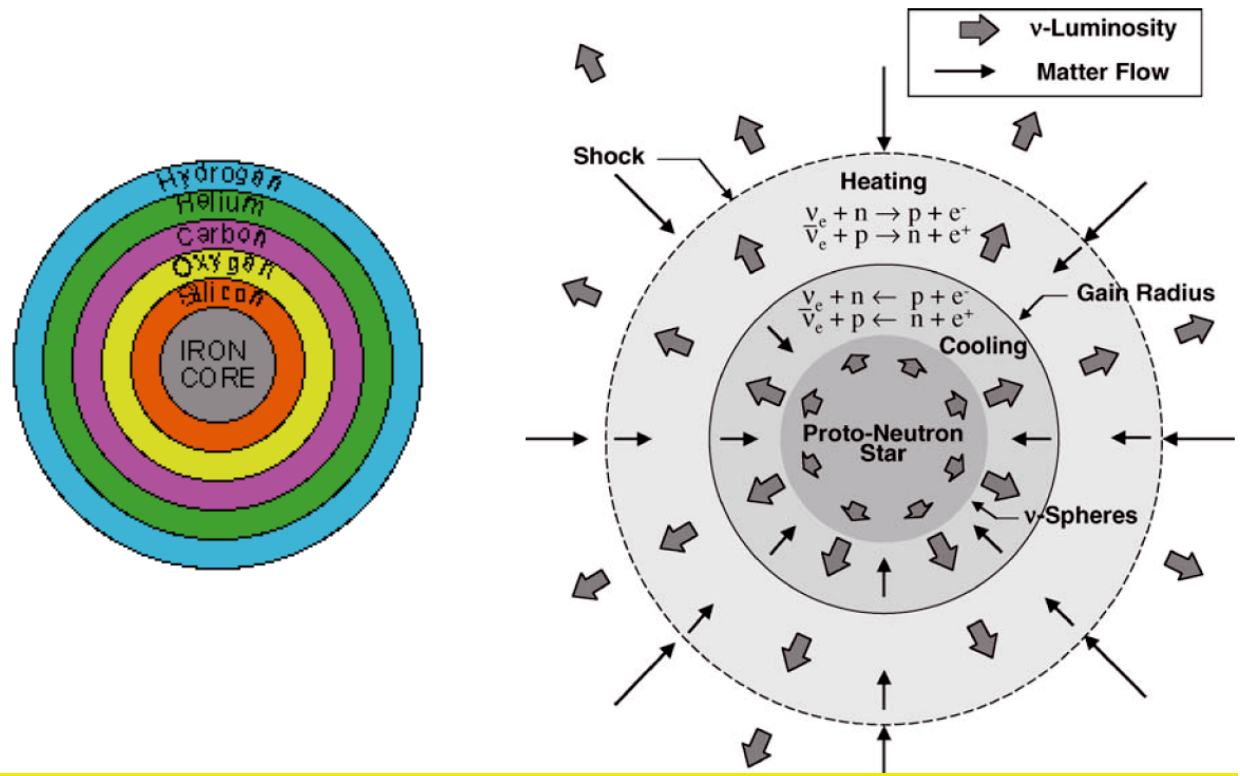

Symmetric nuclear matter at densities of $1-3 \rho_{0}$ 


\section{The Crab nebula ...... and his pulsating heart}

In 1054 chinese astronomers observed a "visiting star": As bright as the full moon for 1 month 1968: discovery of a pulsating radiation source $(30 \mathrm{~Hz})$ in the center of the crab nebula

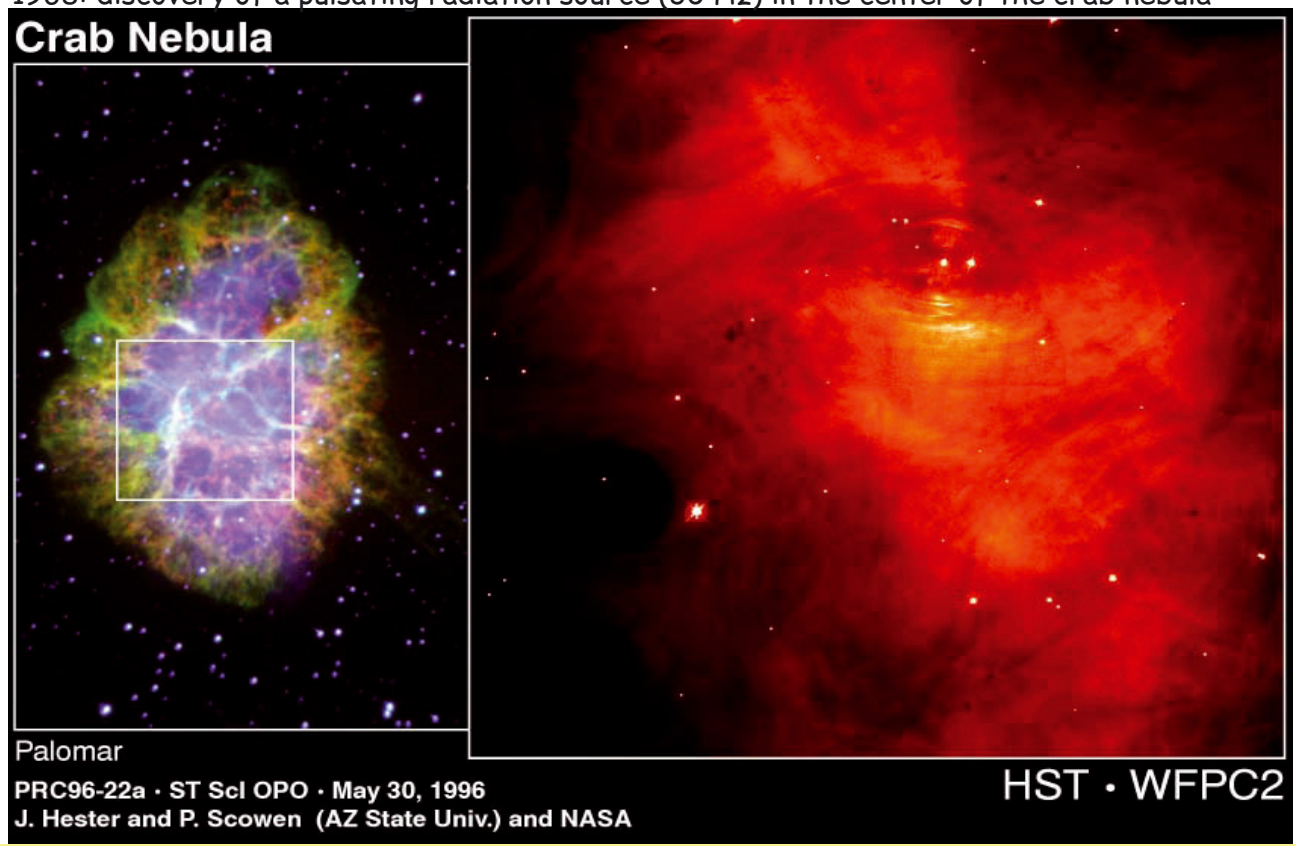

Strongly interacting matter in neutron stars

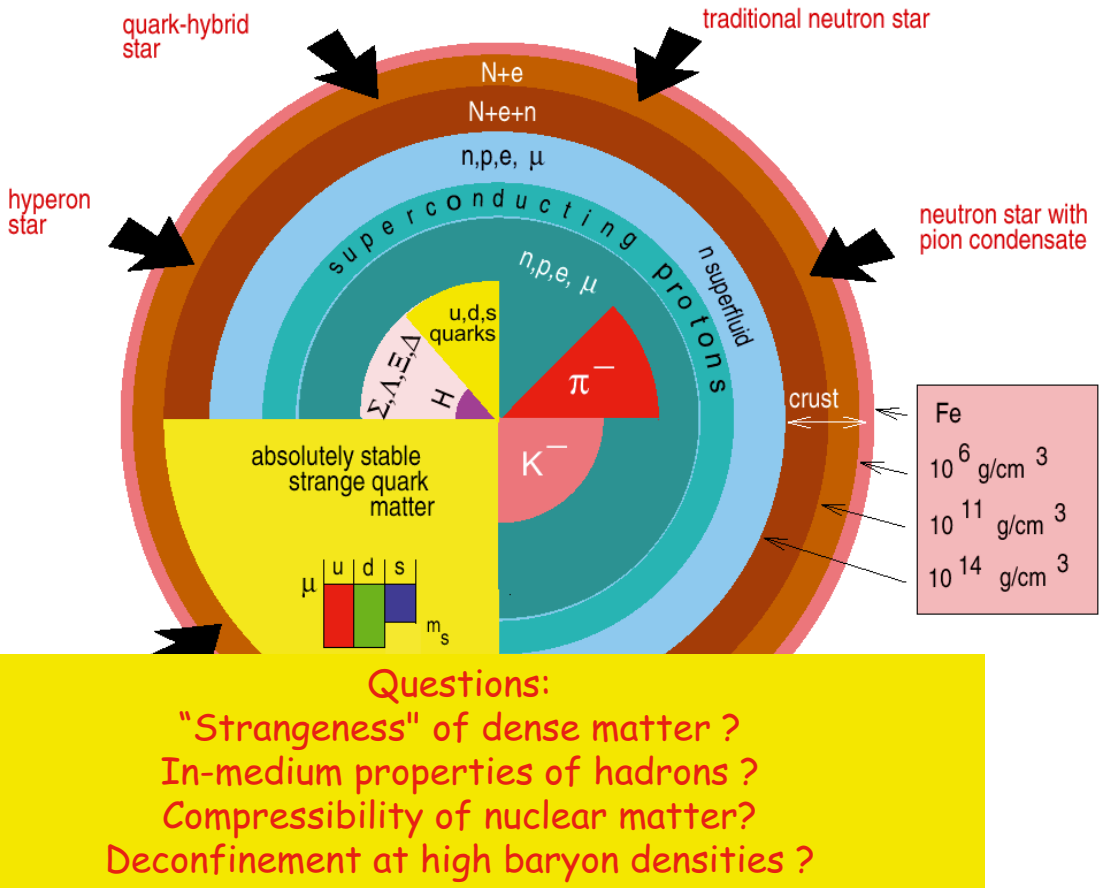




\section{Hyperons in neutron stars}

A. Akmal, V.R. Pandharipande, D.G. Ravenhall, Phys. Rev. C 58 (1998) 1804

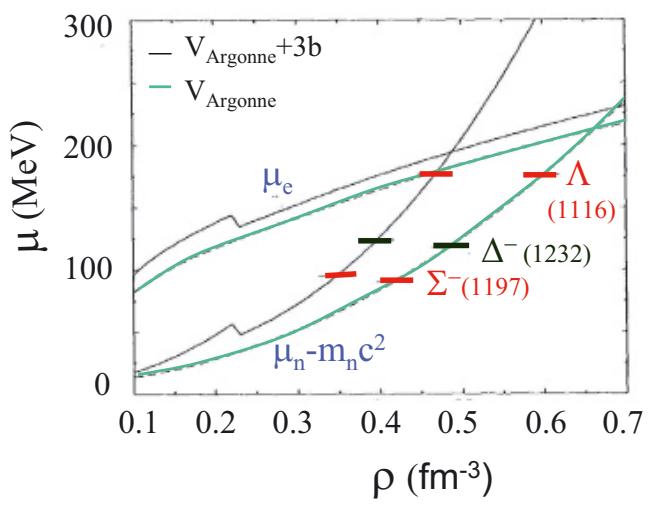

Cold neutral matter in $\beta$-equilibrium: $\mathrm{n}+\mathrm{e}^{-} \Rightarrow \Sigma^{-}+\mathrm{v}_{\mathrm{e}}$

$\mathrm{n} \Rightarrow \Lambda$
N. Glendenning, F. Weber, S. Moszkowski, Phys. Rev. C 45 (1992) 844

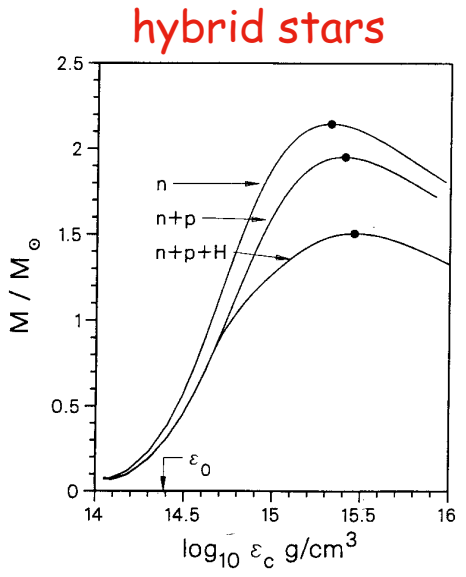

Experiment: Hypernuclei

\section{Antikaons in neutron stars}
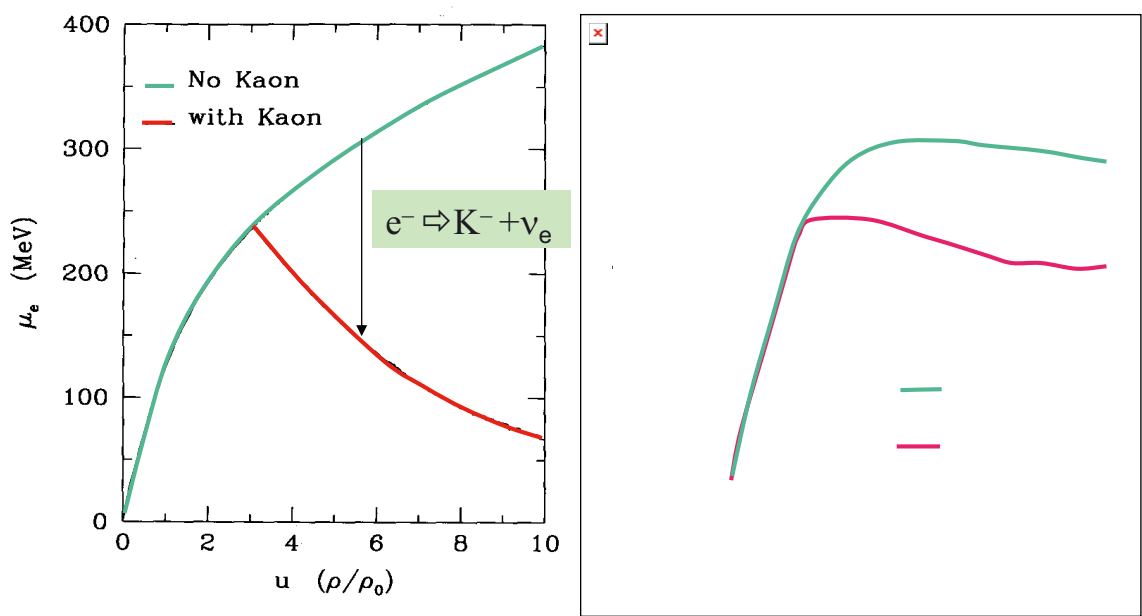

"Kaon condensate": $\mathrm{e}^{-} \Rightarrow \mathrm{K}^{-}+\mathrm{v}_{\mathrm{e}}, \mathrm{n} \Rightarrow \mathrm{p}+\mathrm{K}^{-}$

G.E. Brown, H.A. Bethe, Astrophys. Jour. 423 (1994) 659

G.Q.Li, C.H. Lee, G.E. Brown, Nucl. Phys. A 625 (1997) 


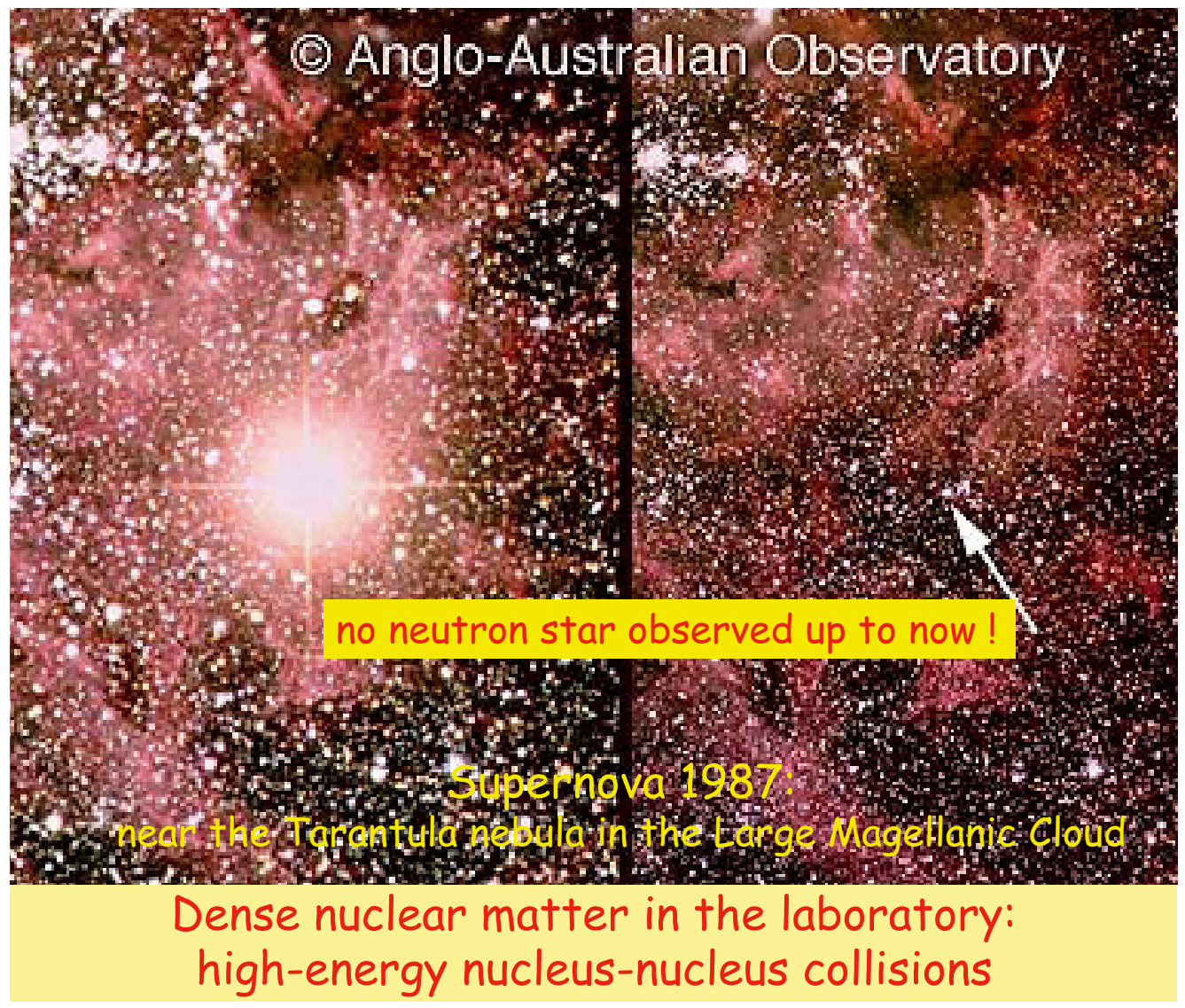

W. Cassing et al., Giessen: Hadron-String Dynamics (HSD): mean field, hadrons + resonances + strings

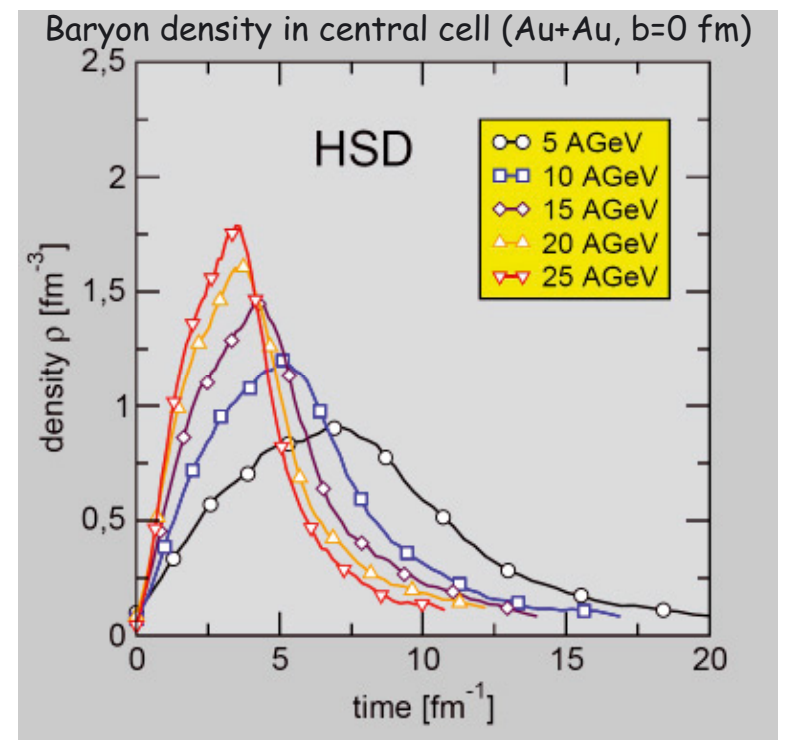


The equation-of-state of (symmetric) nuclear matter

Equation of state:

$P V \propto T \propto E$

$P \propto E / V \propto E / A$

- $E / A\left(\rho_{o}\right)=-16 \mathrm{MeV}$

- $\delta(E / A)\left(\rho_{\mathrm{o}}\right) / \delta \rho=0$

- Compressibility:

$\kappa=9 \rho^{2} \delta^{2}(E / A) / \delta \rho^{2}$

$\kappa=200 \mathrm{MeV}:$ "soft" EOS

$\kappa=380 \mathrm{MeV}:$ "stiff" EOS

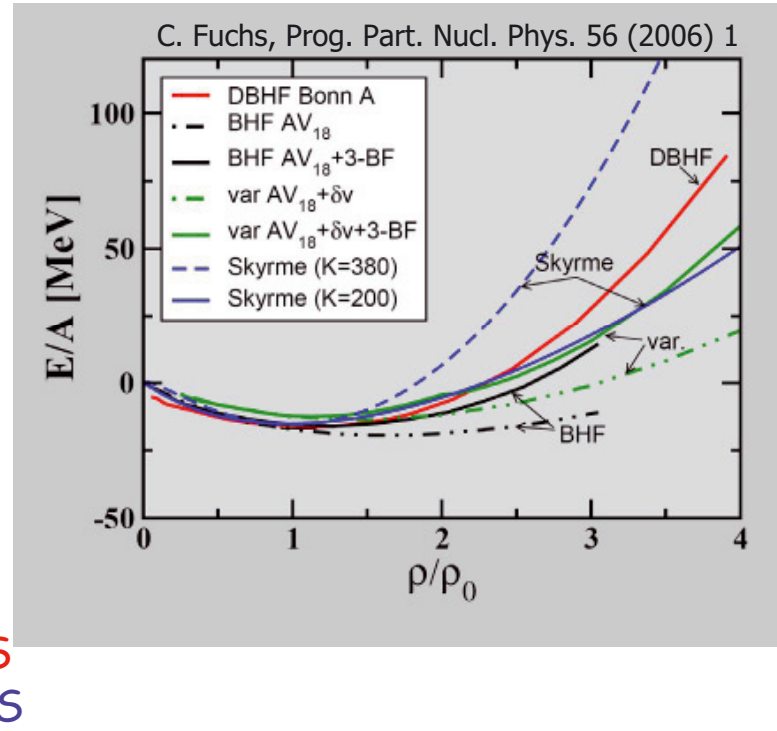

Observable in HI collisions: collective flow (driven by pressure)

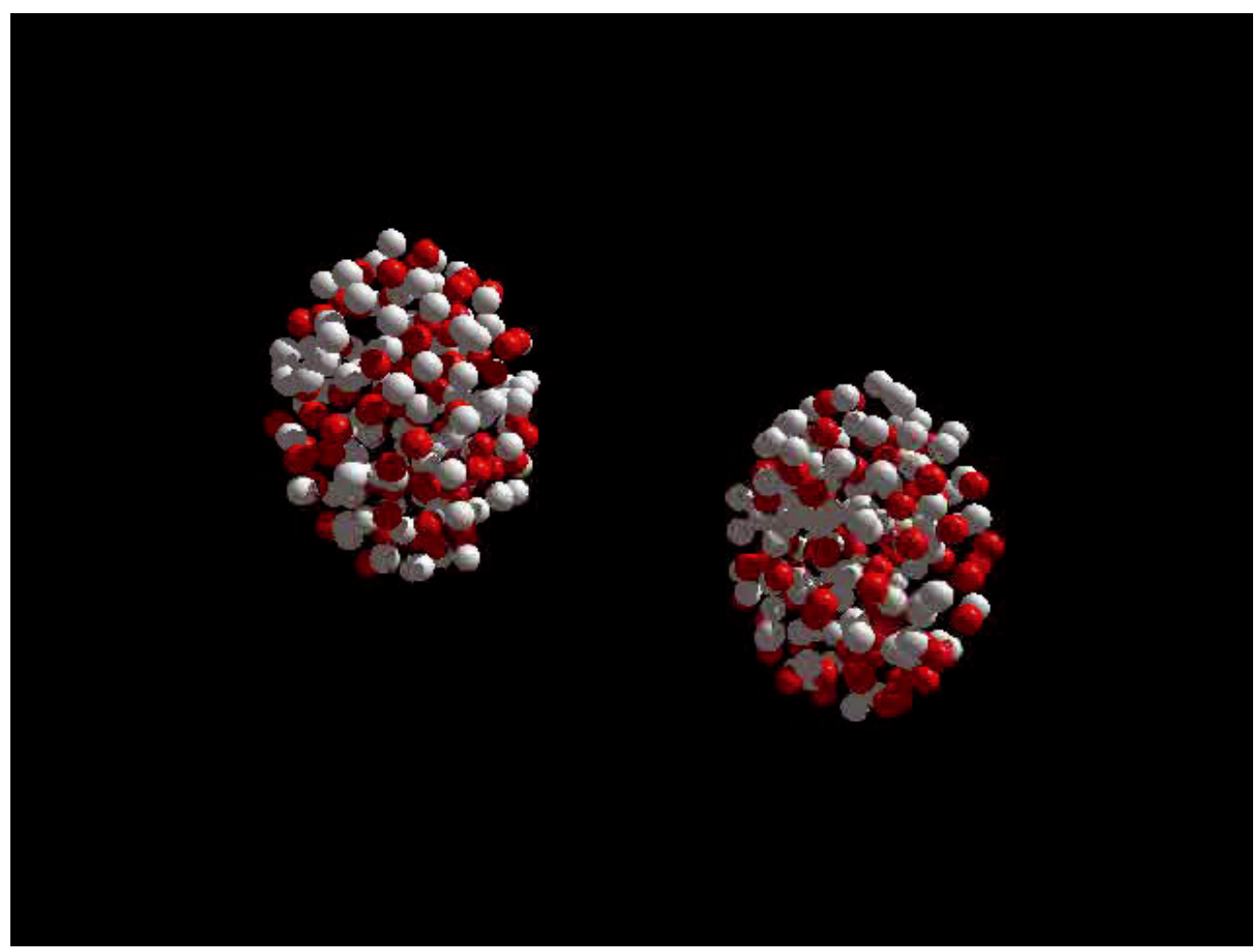




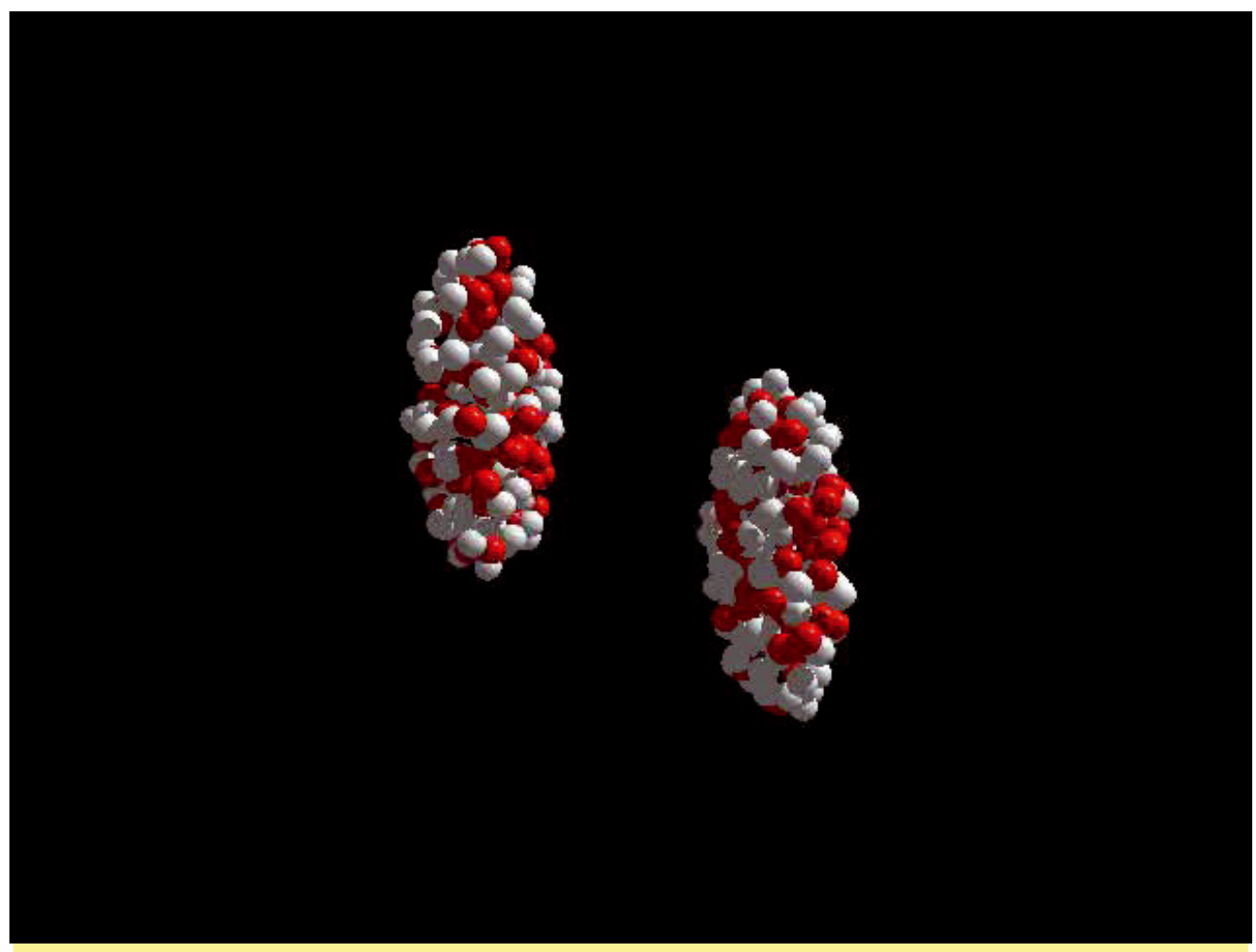

Dynamics of a semi-central Au+Au collision at $2 \mathrm{AGeV}$ (BUU calculation, P. Danielewicz, MSU)

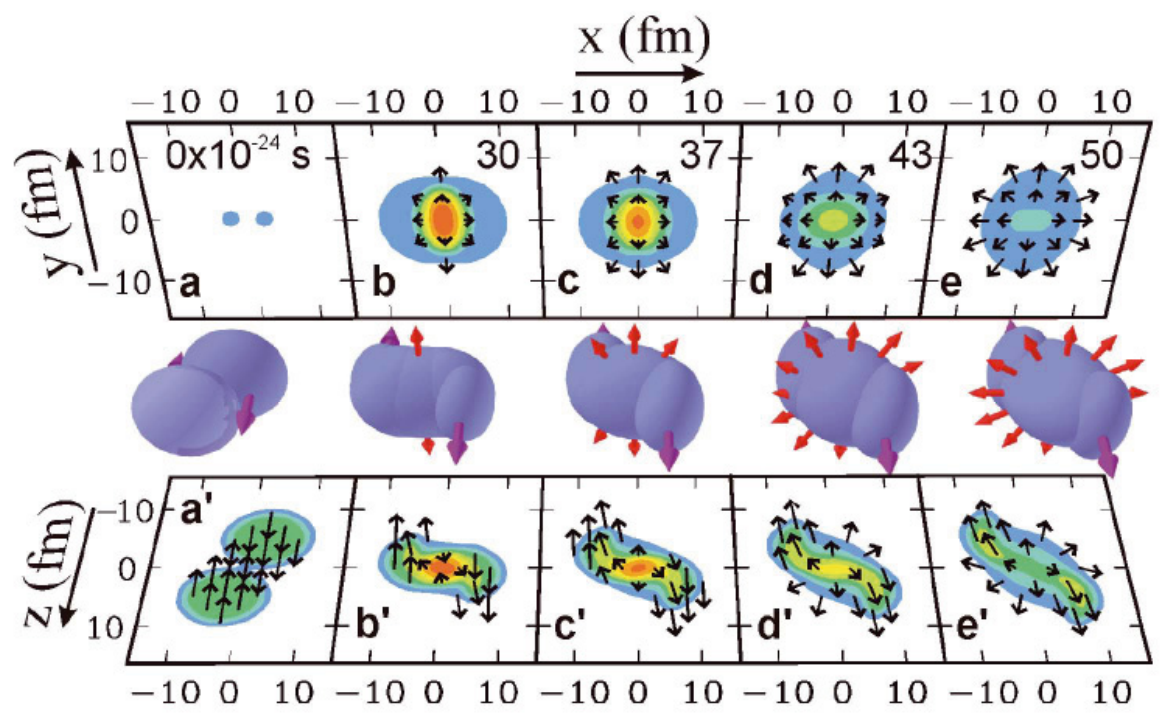

Collective flow of nucleons: driven by pressure 


\section{Experimental determination of collision centrality}
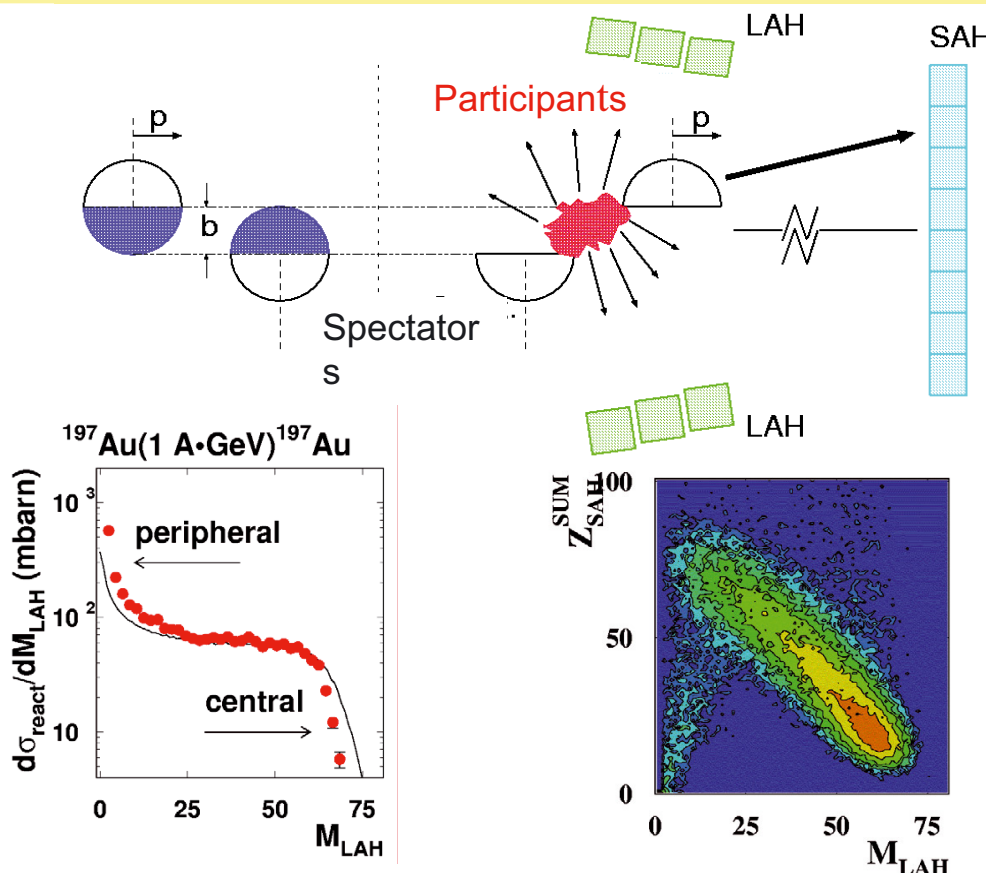

or Zero Degree Calorimeter: $E_{Z D C}=E_{\text {beam }} A_{\text {pro-Spec }}$ and $A_{\text {part }}=2\left(A-E_{Z D C} / E_{\text {beam }}\right)$ Experimental determination of the reaction plane
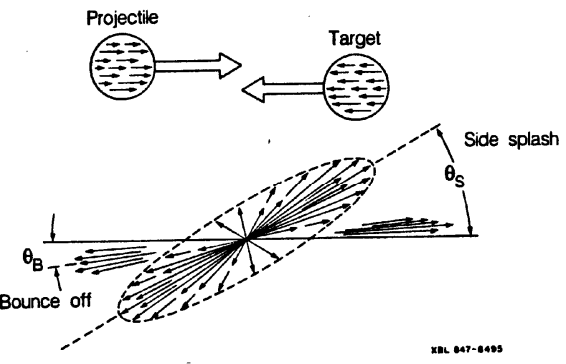

Transverse Momentum Method:

P. Danielewicz \& G. Odyniec, Phys. Lett. 157 B (1985) 146

$\mathbf{Q}=\Sigma_{v} \omega \cdot \mathbf{p}_{v}$

$\omega=1$ für $\mathrm{y}>\mathrm{y}_{\mathrm{cm}}$

$\Phi_{\mathrm{R}}=\arctan \left(\mathrm{Q}_{\mathrm{y}} / \mathrm{Q}_{\mathrm{x}}\right)$

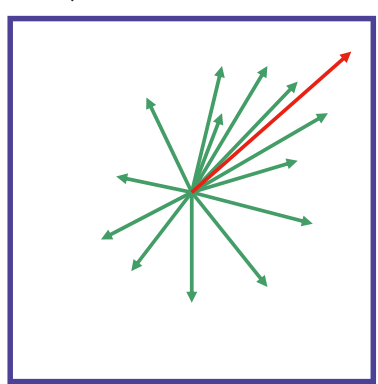

Dispersion of the reaction plane: Sub-Event-Method: $\Delta \Phi=\Phi_{1}-\Phi_{2}$

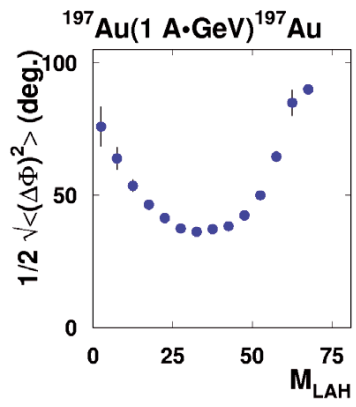




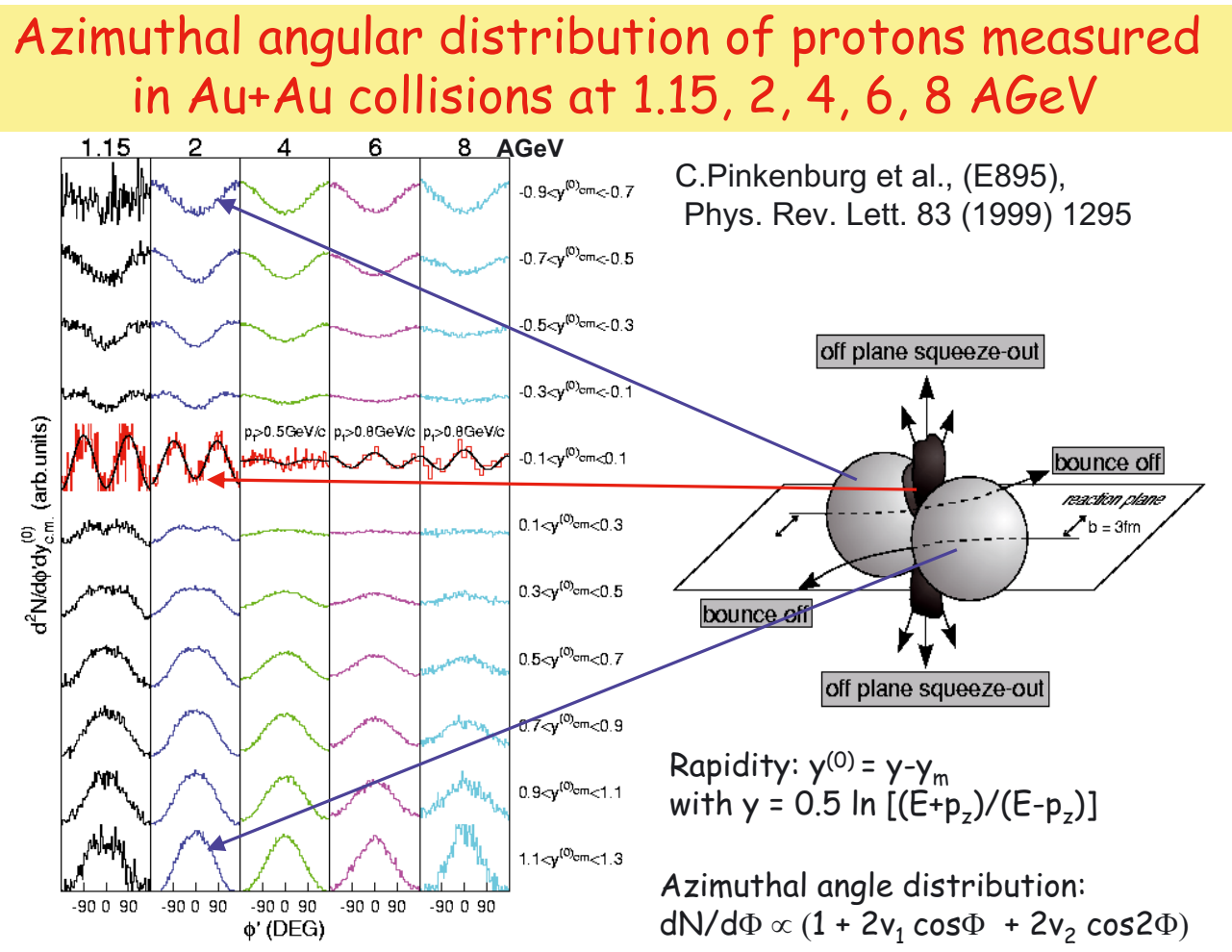

\section{Compressibility extracted from collective flow}

P. Danielewicz, R. Lacey, W.G. Lynch, Science 298 (2002) 1592

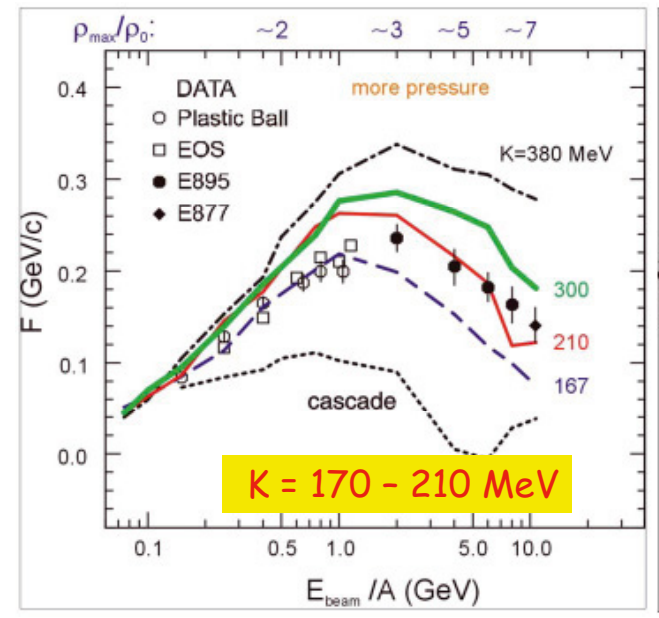

Transverse in-plane flow:

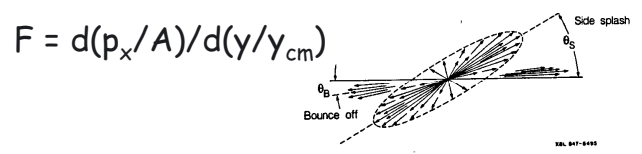

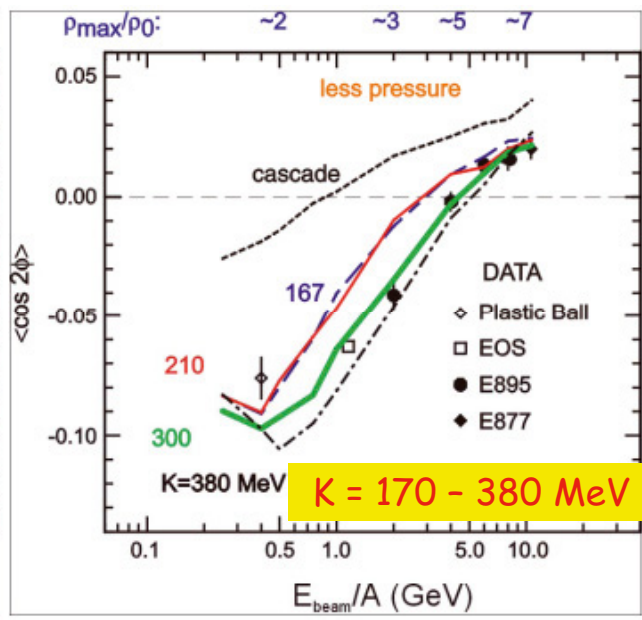

Elliptic flow:

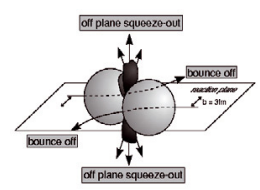

$\mathrm{dN} / \mathrm{d} \Phi \propto\left(1+2 \mathrm{v}_{1} \cos \Phi+2 \mathrm{v}_{2} \cos 2 \Phi\right)$ 


\section{New data: Au + Au collisions at SIS energies}

A. Andronic et al. (FOPI Collaboration) Phys. Lett. B612 (2005) 173
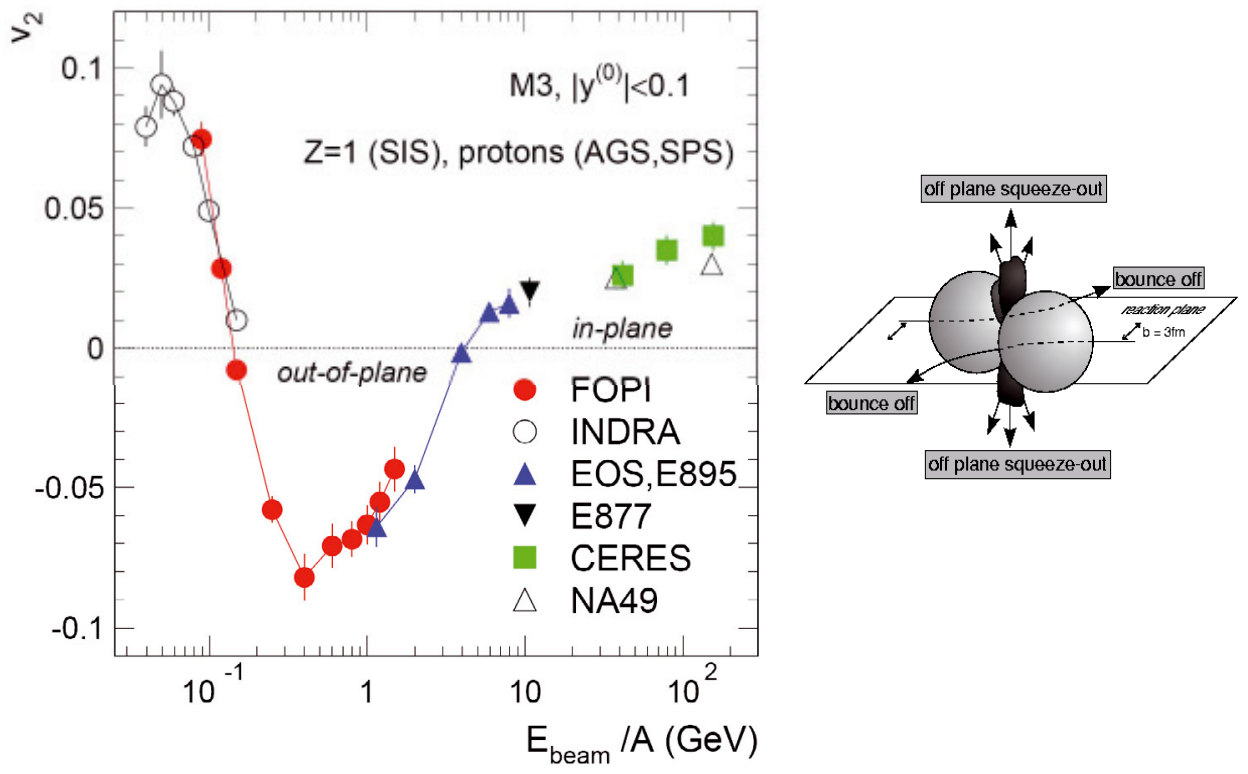

\section{Summary: Collective proton flow in Au+Au collisions and the nuclear matter EOS}

\begin{tabular}{|c|c|c|c|}
\hline $\begin{array}{c}\text { Beam energy } \\
\text { AGeV }\end{array}$ & central density & flow observable & $\begin{array}{c}\text { compressibility } \\
\text { K }\end{array}$ \\
\hline $0.4-1.5$ & $\rho=1-3 \rho_{0}$ & transverse, elliptic & $170-210 \mathrm{MeV}$ \\
\hline $2-10$ & $\rho=3-5(7) \rho_{0}$ & transverse & $170-210 \mathrm{MeV}$ \\
\hline $2-10$ & $\rho=3-5(7) \rho_{0}$ & elliptic & $300-380 \mathrm{MeV}$ \\
\hline
\end{tabular}

Within microscopic transport models the collective flow is sensitive to:

$>$ The nuclear matter equation of state

In-medium nucleon-nucleon cross sections

$>$ Momentum dependent interactions 


\section{Kaon production in $\mathrm{Au}+\mathrm{Au}$ collisions at $1 \mathrm{AGeV}$ $\mathrm{pp} \rightarrow \mathrm{K}^{+} \wedge \mathrm{p} \quad\left(E_{\text {thres }}=1.6 \mathrm{GeV}\right)$}

RBUU: $A u+A u 1 A G e V, b=0 \mathrm{fm}$

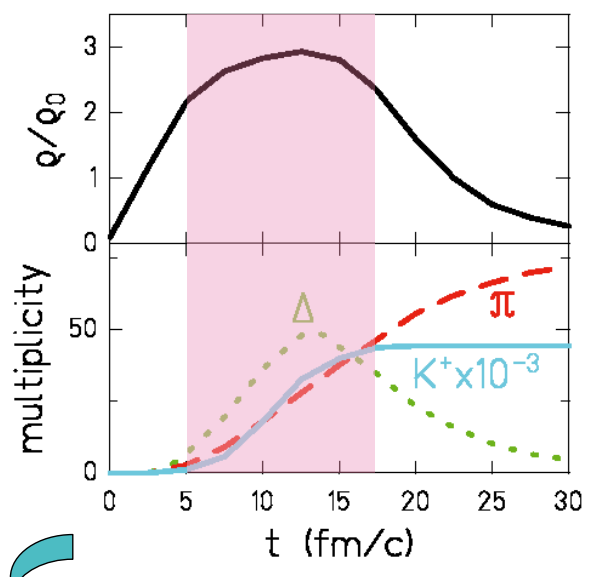

$\mathrm{K}^{+}$mesons probe high densities

$\mathrm{K}^{+}$mesons scatter elastically only
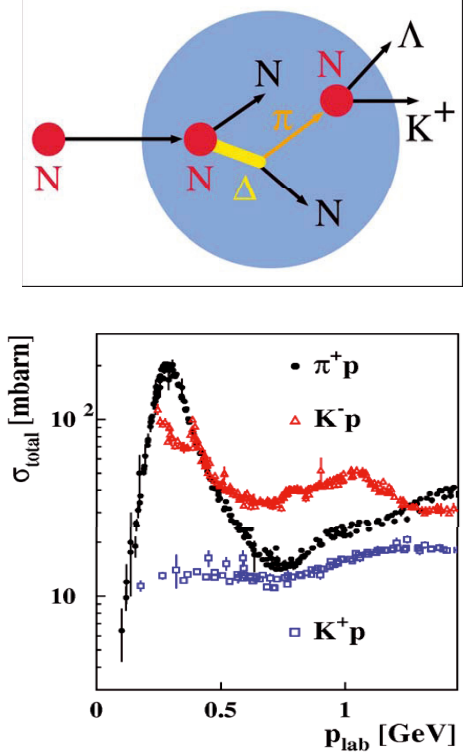

Probing the nuclear equation-of-state $\left(\rho=1-3 \rho_{0}\right)$ by $\mathrm{K}^{+}$meson production in $\mathrm{C}+\mathrm{C}$ and $\mathrm{Au}+\mathrm{Au}$ collisions

Idea: $\mathrm{K}^{+}$yield $\propto$ baryon density $\rho \propto$ compressibility $\mathrm{K}$

Transport model (RBUU)

$A u+A u$ at 1 AGeV:

$K=200 \mathrm{MeV} \Rightarrow \rho_{\max } \approx 2.9 \rho_{0} \Rightarrow K^{+} \hat{y}$

$K=380 \mathrm{MeV} \Rightarrow \rho_{\max } \approx 2.4 \rho_{0} \Rightarrow K+2 \nabla$

Reference system $C+C$ :

$\mathrm{K}^{+}$yield not sensitive to EOS

Experiment:

C. Sturm et al., (KaoS Collaboration), Phys. Rev. Lett. 86 (2001) 39

Theory:

Ch. Fuchs et al.,

Phys. Rev. Lett. 86 (2001) 1974

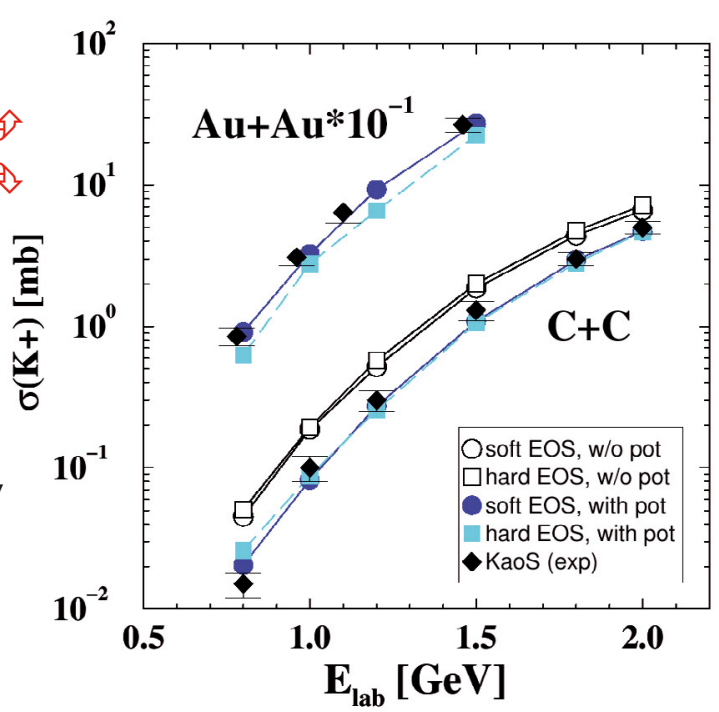




\section{The compressibility of nuclear matter}

Experiment: C. Sturm et al., (KaoS Collaboration) Phys. Rev. Lett. 86 (2001) 39 Theory: QMD Ch. Fuchs et al., Phys. Rev. Lett. 86 (2001) 1974

IQMD Ch. Hartnack, J. Aichelin, J. Phys. G 28 (2002) 1649
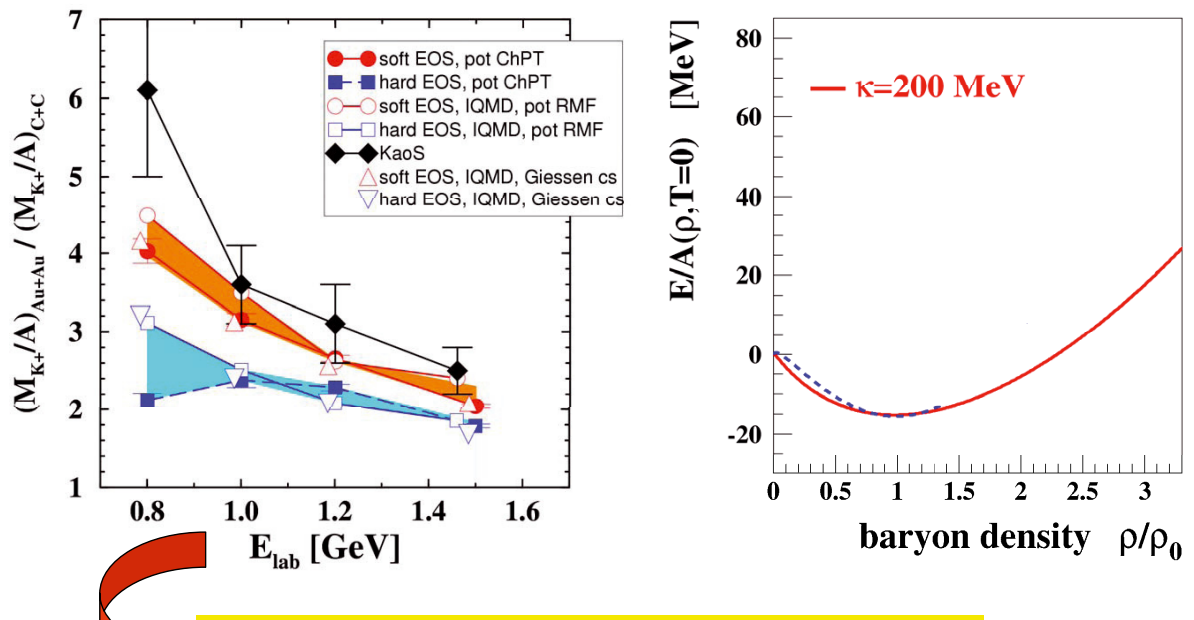

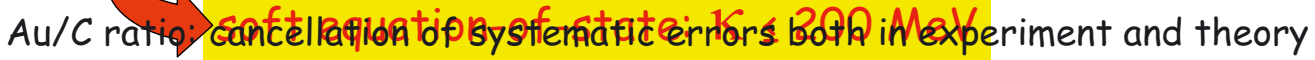

Outlook: determination of the nuclear EOS at very high $\rho$

-

Exploring the "nuclear" EOS at $3 \rho_{0}<\rho<7 \rho_{0}$

Measure excitation function of (multi-strange) hyperon production in heavy-ion collisions from 2 - $15 \mathrm{AGeV}$ (no data yet):

Direct production:

$\mathrm{NN} \rightarrow \Lambda^{0} \overline{\Lambda^{0}} \mathrm{NN} \quad\left(\mathrm{E}_{\text {thr }}=7.1 \mathrm{GeV}\right)$

$\mathrm{NN} \rightarrow \Xi^{+} \Xi^{-} \mathrm{NN} \quad\left(\mathrm{E}_{\text {thr }}=9.0 \mathrm{GeV}\right)$

$\mathrm{NN} \rightarrow \Omega^{+} \Omega^{-} \mathrm{NN} \quad\left(\mathrm{E}_{\mathrm{thr}}=12.7 \mathrm{GeV}\right)$

Production via multiple collisions:

$\mathrm{NN} \rightarrow \mathrm{K}^{+} \Lambda^{0} \mathrm{~N}, \quad \mathrm{NN} \rightarrow \mathrm{K}^{+} \mathrm{K}^{-} \mathrm{NN}, \quad \Lambda^{0} \mathrm{~K}^{-} \rightarrow \Xi^{-} \pi^{0}, \quad \Xi^{-} \mathrm{K}^{-} \rightarrow \Omega^{-} \pi^{-}$ $\overline{\Lambda^{0}} \mathrm{~K}^{+} \rightarrow \Xi^{+} \pi^{0}, \Xi^{+} \mathrm{K}^{+} \rightarrow \Omega^{+} \pi^{+}$. 


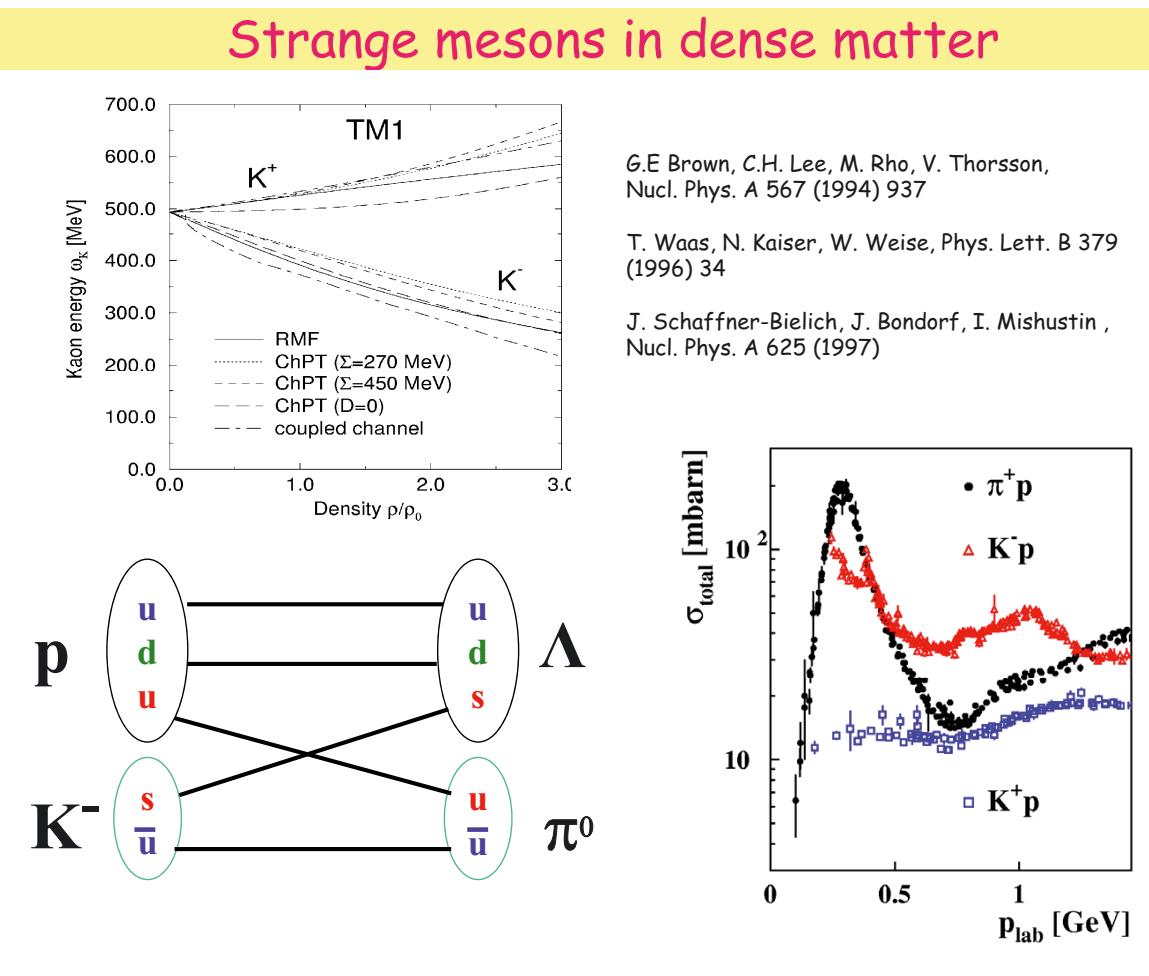

\section{In-medium modifications of $\mathrm{K}^{+}$mesons}

Data: M. Menzel et al., (KaoS Collab.), Phys. Lett. B 495 (2000) 26

K. Wisniewski et al., ( FOPI Collab.), Eur. Phys. J A 9 (2000) 515

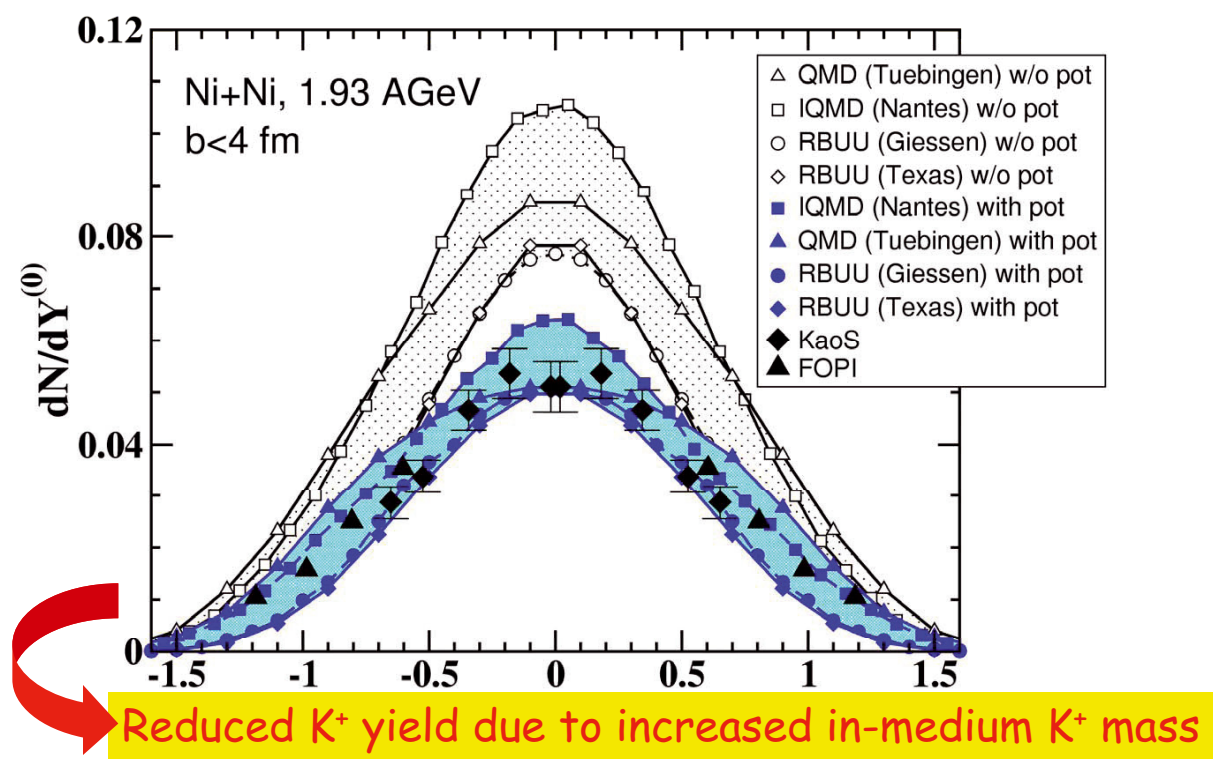




\section{$\mathrm{K}^{+}$azimuthal emission pattern from $A+A$ collisions}

Data: Y. Shin et al., (KaoS Collaboration), Phys. Rev. Lett. 81 (1998) 1576

F. Uhlig et al., (KaoS Collaboration), Phys. Rev. Lett. 95 (2005) 012301

Calculations see A. Larionov, U. Mosel, nucl-th/0504023

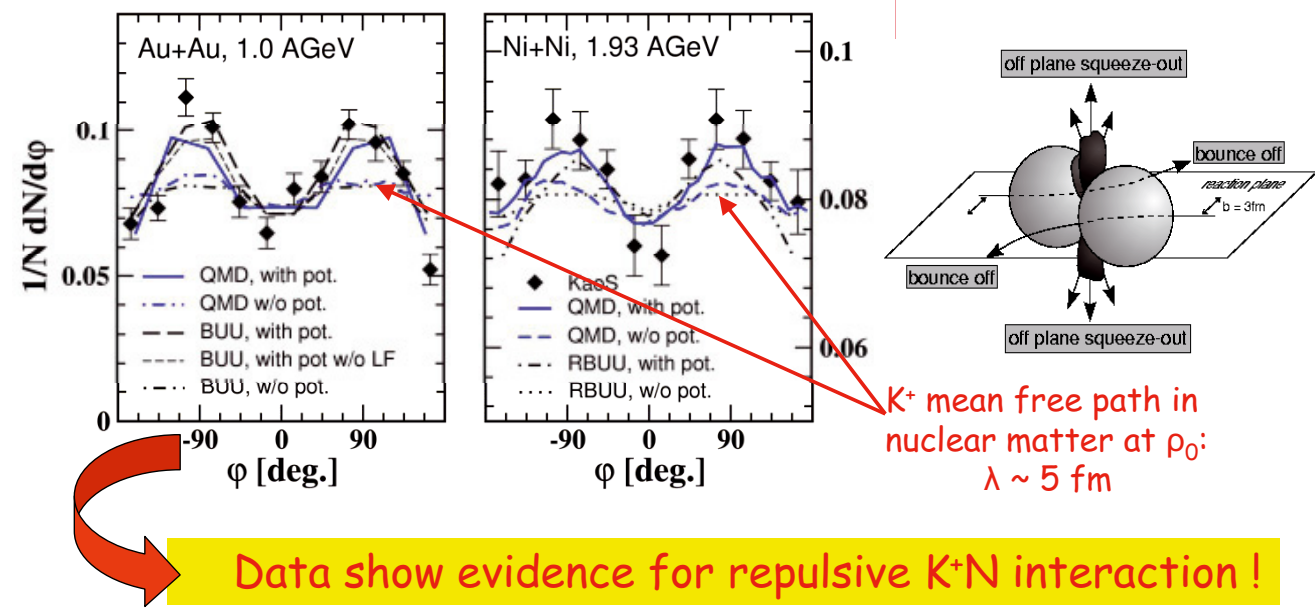

\section{$\mathrm{Ni}+\mathrm{Ni}$ at $1.93 \mathrm{AGeV}: \pi, \mathrm{K}^{+}$and $\mathrm{K}^{-}$azimuthal distributions}

F. Uhlig et al., (KaoS Collaboration), Phys. Rev. Lett. 95 (2005) 012301
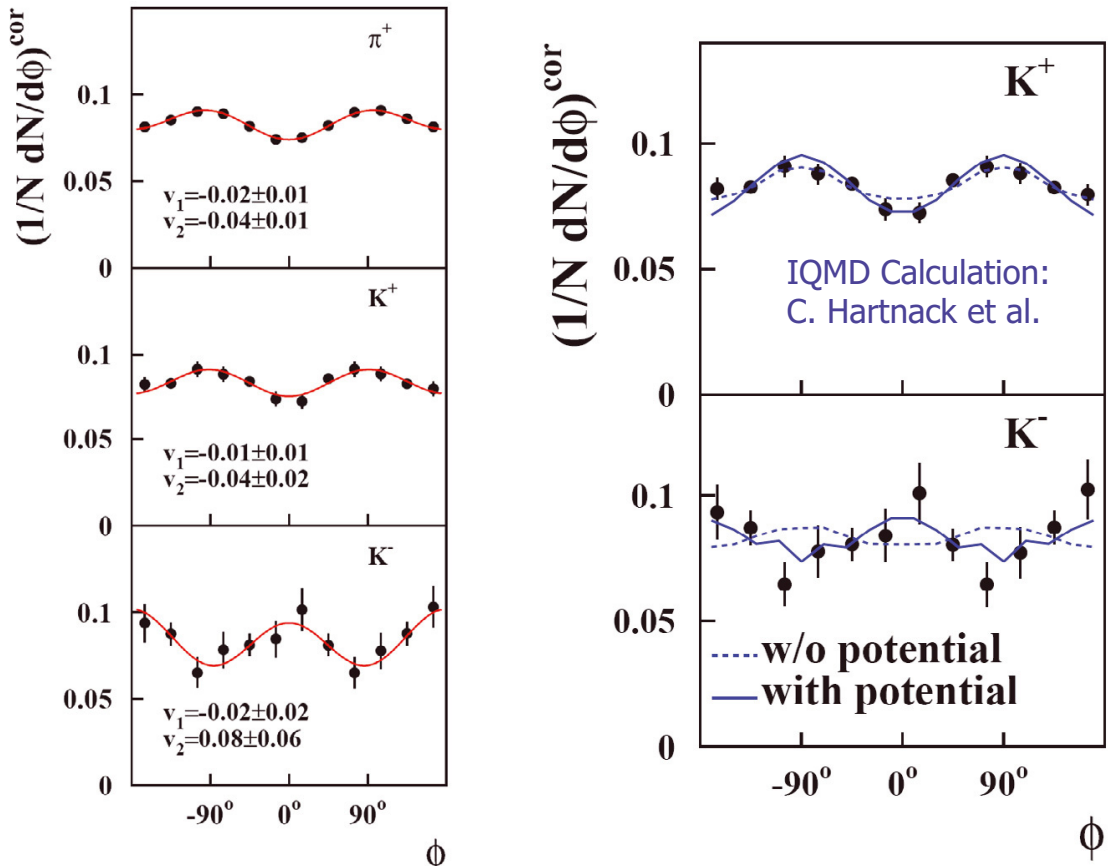


\section{$A u+A u 1.5$ AGeV semi-central collisions ( $b>6.4 \mathrm{fm}$ ) $\mathrm{K}^{+}$and $\mathrm{K}^{-}$azimuthal angular distributions}

M. Płoskon, PhD Thesis 2005
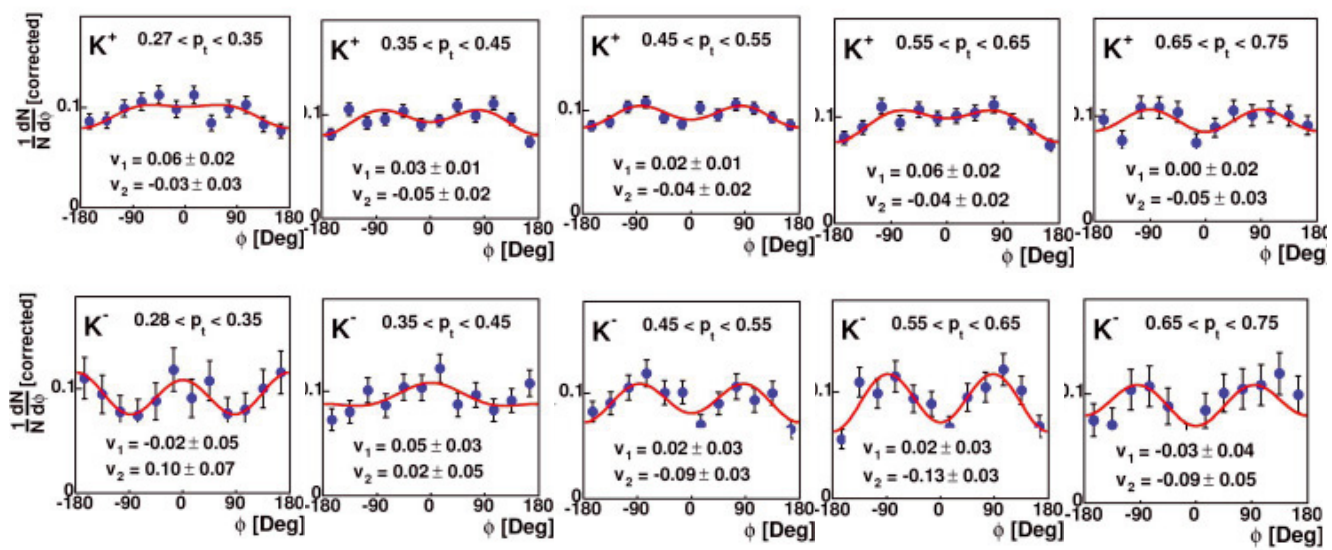

$$
\mathrm{dN}(\varphi) / \varphi \propto 1+2 \mathrm{v}_{1} \cos (\varphi)+2 \mathrm{v}_{2} \cos (2 \varphi)+\ldots
$$

\section{Elliptic flow of $\mathrm{K}^{+}$and $\mathrm{K}^{-}$mesons: Comparison to off-shell transport calculations and in-medium spectral functions}

Data: M. Płoskon, PhD Thesis, Univ. Frankfurt 2005

Off-shell transport calculations: W. Cassing et al., NPA 727 (2003) 59, E. Bratkovskaya, priv. com. Coupled channel G-Matrix approach (K- spectral functions): L. Tolos et al., NPA 690 (2001) 547

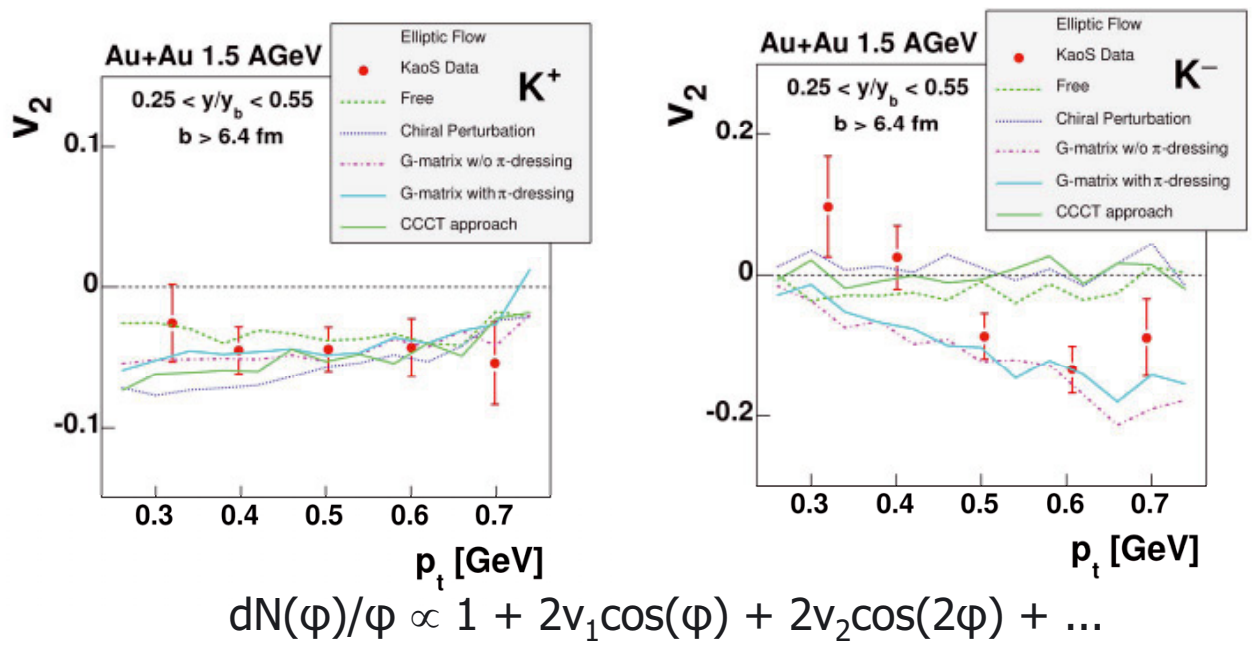




\section{Antikaon spectral function in nuclear matter}
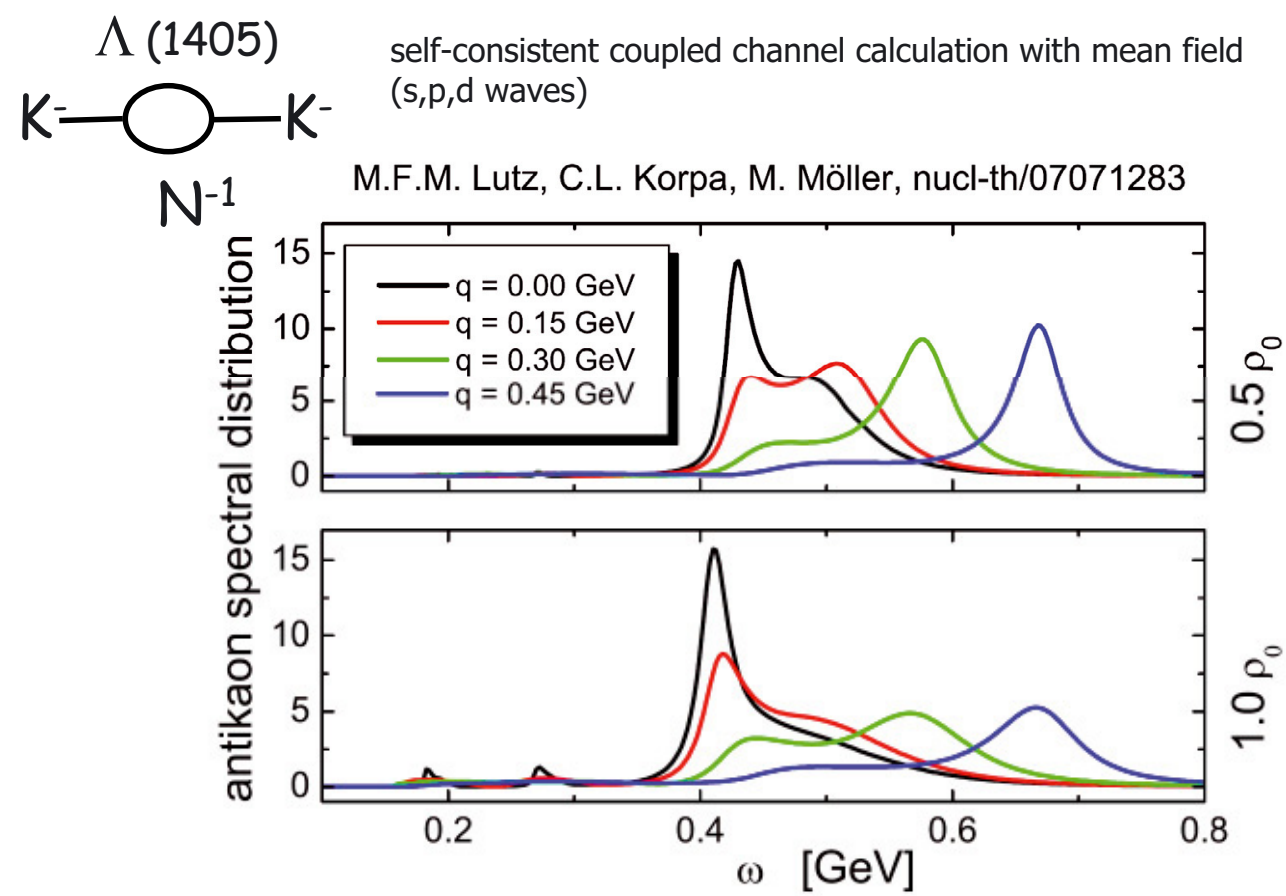

Strangeness production in proton - nucleus collisions

$$
\begin{aligned}
& p+C \rightarrow K^{+}+X \quad(1.6,2.5,3.5 \mathrm{GeV}) \\
& p+C \rightarrow K^{+}+X \quad(2.5,3.5 \mathrm{GeV}) \\
& p+A u \rightarrow K^{+}+X \quad(1.6,2.5,3.5 \mathrm{GeV}) \\
& p+A u \rightarrow K^{-}+X \quad(2.5,3.5 \mathrm{GeV})
\end{aligned}
$$

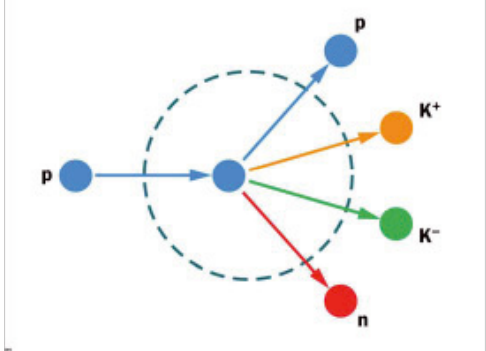

W. Scheinast et al., (KaoS Collaboration) Phys. Rev. Lett. 96 (2006) 072301

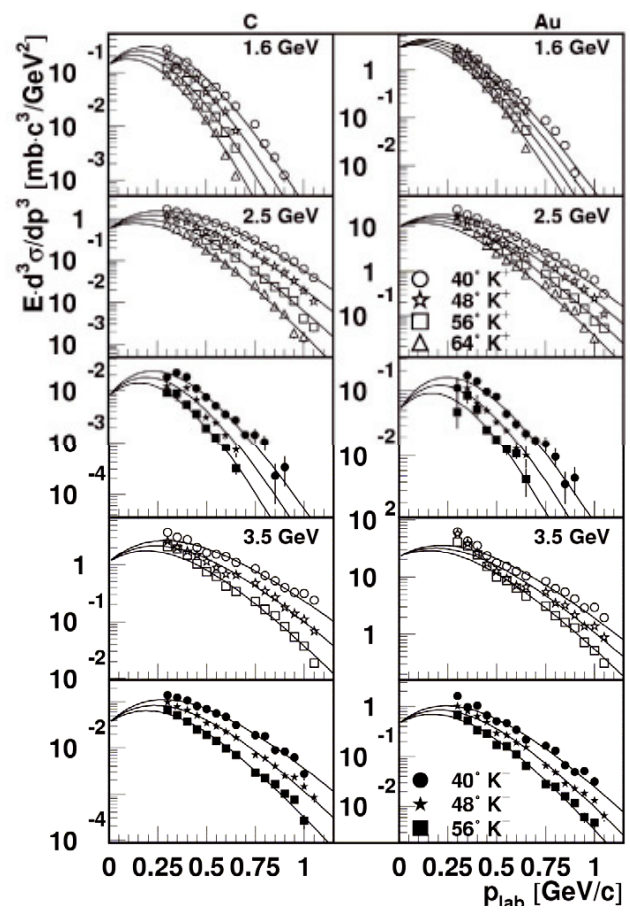




\section{Comparison of $p+A$ data to transport calculations}

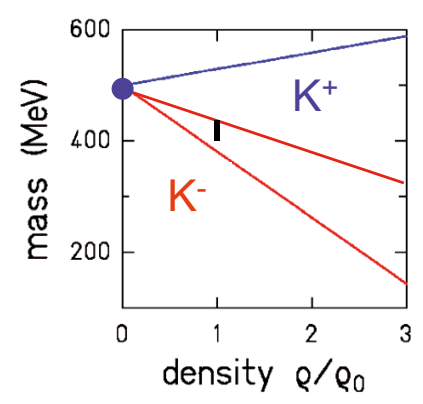

contributing channels:

$\mathrm{p}+\mathrm{N} \rightarrow \mathrm{K}^{+}+\Lambda$

$\mathrm{p}+\mathrm{N} \rightarrow \mathrm{N}+\mathrm{N}+\mathrm{K}^{+}+\mathrm{K}^{-}$

$\Lambda+\mathrm{N} \rightarrow \mathrm{N}+\mathrm{N}+\mathrm{K}^{-}$

(strangeness exchange)

Transport calculation: H. W. Barz et al., Phys.Rev. C68 (2003) 041901

\section{Summary Kaon production}

Excitation function of $\mathrm{K}^{+}$production in $A+A$ collisions $\left(\rho=1-3 \rho_{0}\right)$ :

$\Leftrightarrow$ The nuclear matter equation-of-state is soft $(K \approx 200 \mathrm{MeV})$

Yield and elliptic flow of $K^{+}$mesons in $A+A$ collisions:

$\Leftrightarrow$ The in-medium potential of $K+$ mesons is repulsive

Yield of $K$ - mesons proton-nucleus collisions:

$\leftrightarrow$ Evidence for a K-N in-medium potential of $U_{K} \approx-80 \rho / \rho_{0} \mathrm{MeV}$

Yield and elliptic flow of $K$ - mesons in $A+A$ collisions:

$\Leftrightarrow$ Quantitative interpretation of data requires off-shell transport calculations and in-medium spectral functions 


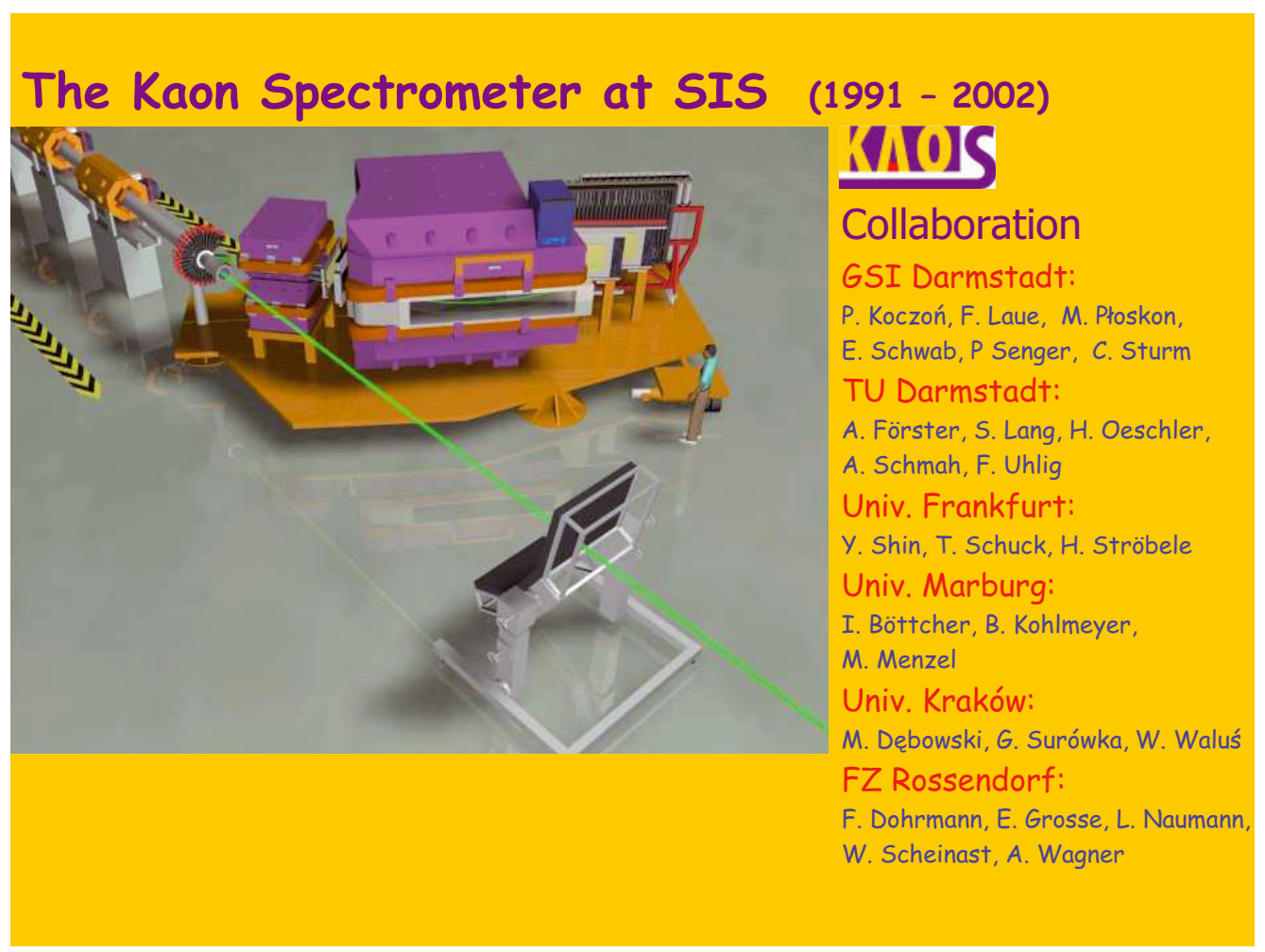

Facility for Antiproton and Ion Research (FAIR)

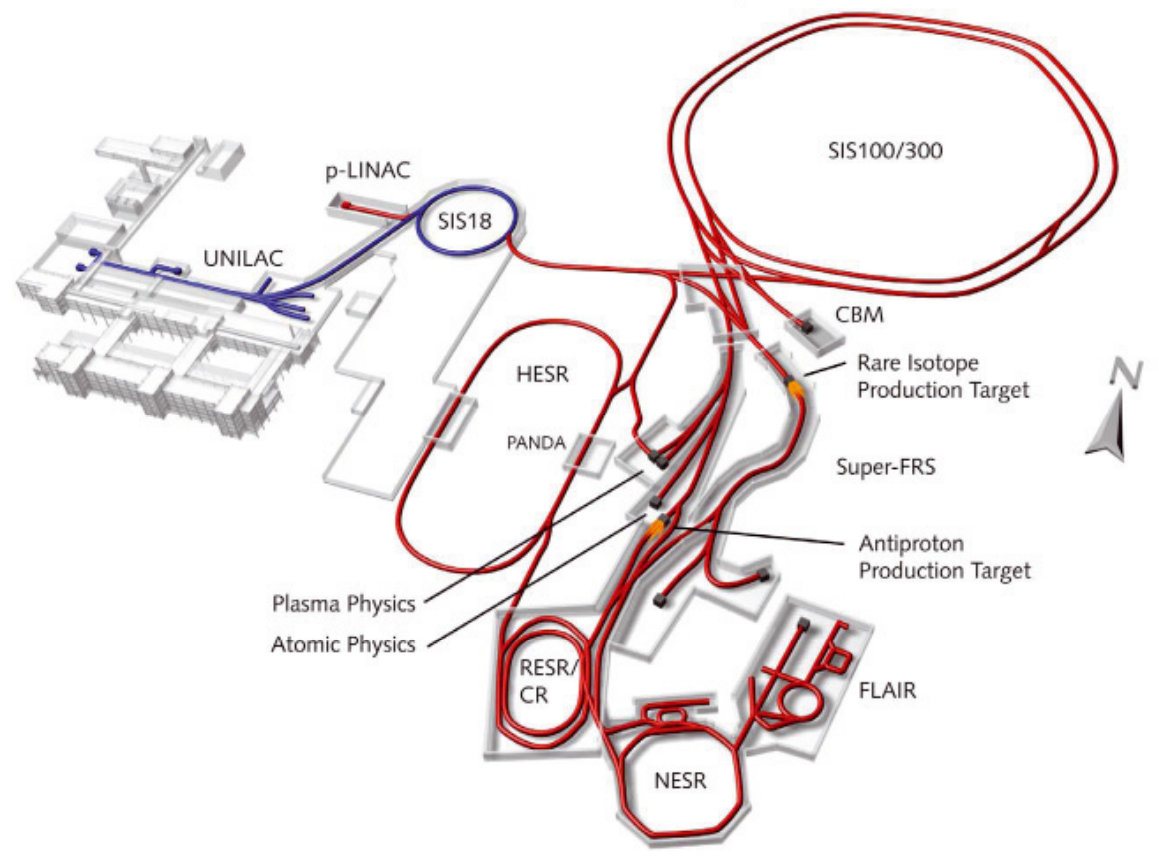




\title{
Compressed Baryonic Matter
}

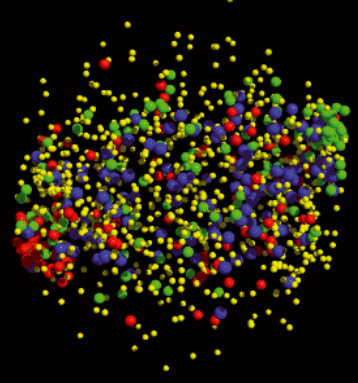

\author{
Peter Senger (GSI)
}

Towards highest baryon densities:

$>$ Exploring the phases of QCD matter

Dense Matter in HIC and Astrophysics, 14. - 26. 07. 2008, Dubna, Russia

\section{Facility for Antiproton and Ion Research (FAIR)}

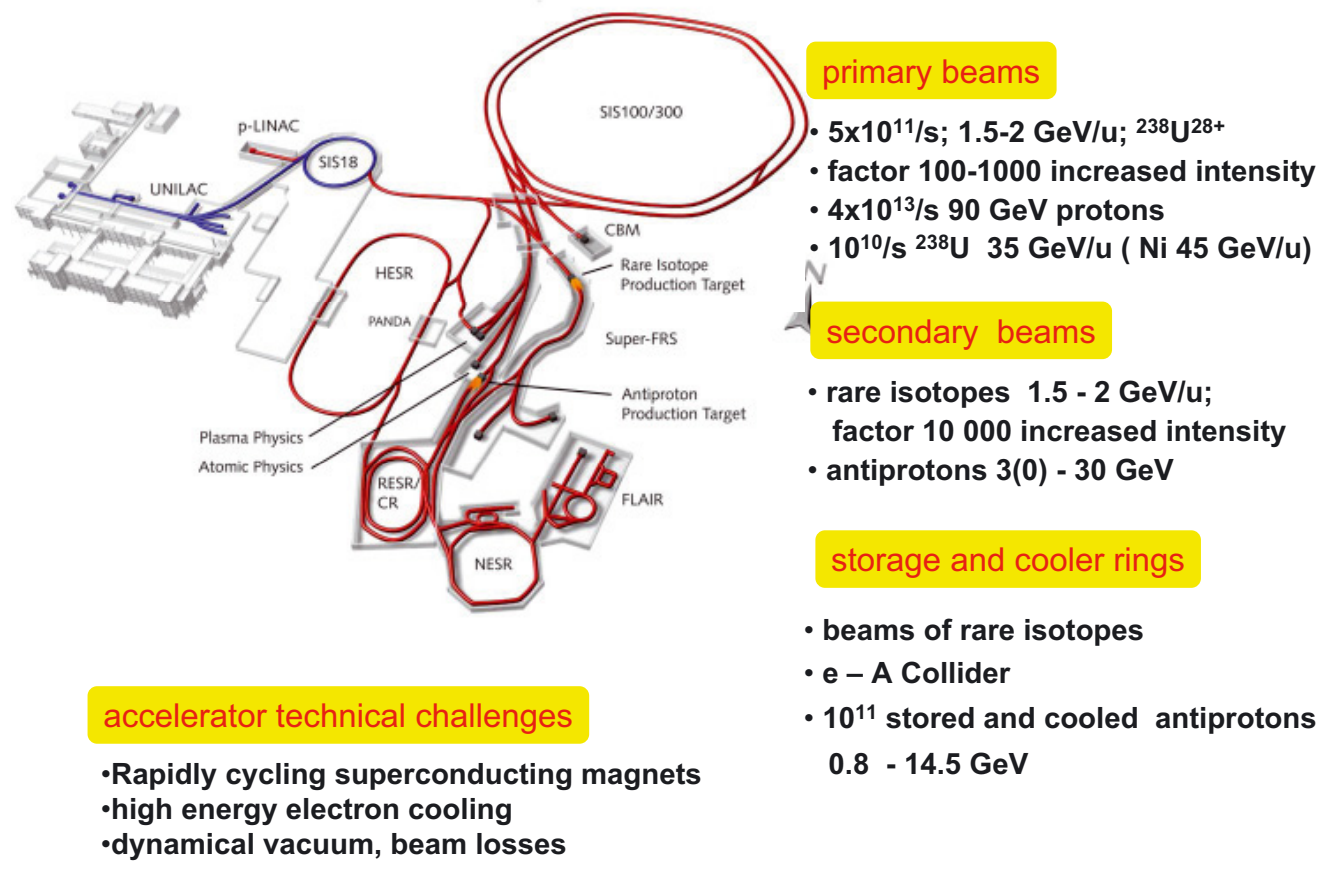




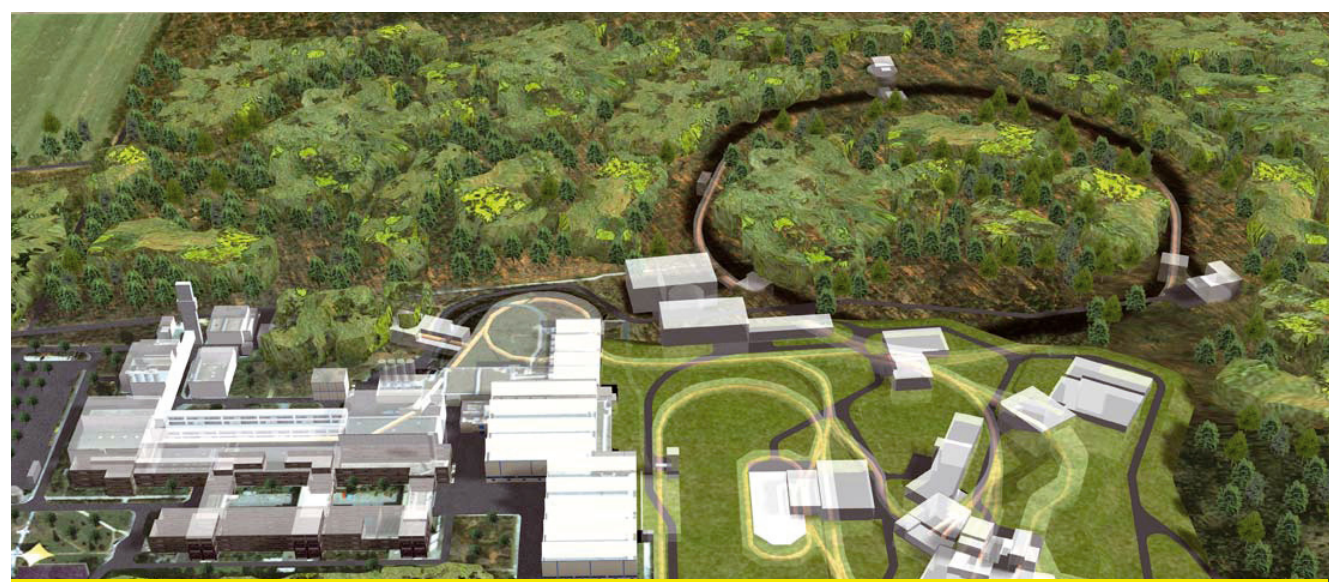

Cost of the FAIR project: 1.2 Billion $€$ (25\% from foreign partners).

15 FAIR member states:

Austria, China, Finland, France, Germany, Great Britain, Greece, India, Italy, Poland, Romania, Russia, Slovenia, Spain, Sweden,

German Federal Government has approved budget over 10 years FAIR project start: Nov. 2007

First beams planned for $2014-2015$

\section{Research programs at FAIR}

Rare isotope beams; nuclear structure and nuclear astrophysics nuclear structure far off stability nucleosynthesis in stars and supernovae

Beams of antiprotons: hadron physics quark-confinement potential search for gluonic matter and hybrids hypernuclei

high-energy nucleus-nucleus collisions: compressed baryonic matter baryonic matter at highest densities (neutron stars) phase transitions and critical endpoint in-medium properties of hadrons

short-pulse heavy ion beams: plasma physics matter at high pressure, densities, and temperature fundamentals of nuclear fusion

atomic physics and applied research highly charged atoms low energy antiprotons radiobiology

accelerator physics

high intensive heavy ion beams dynamical vacuum rapidly cycling superconducting magnets high energy electron cooling
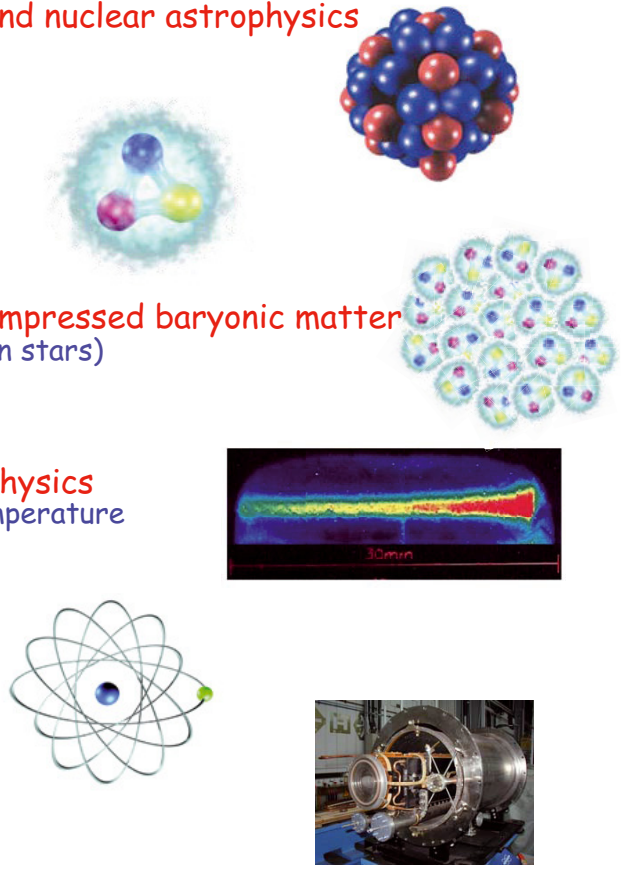


\section{Exploring the QCD phase diagram}

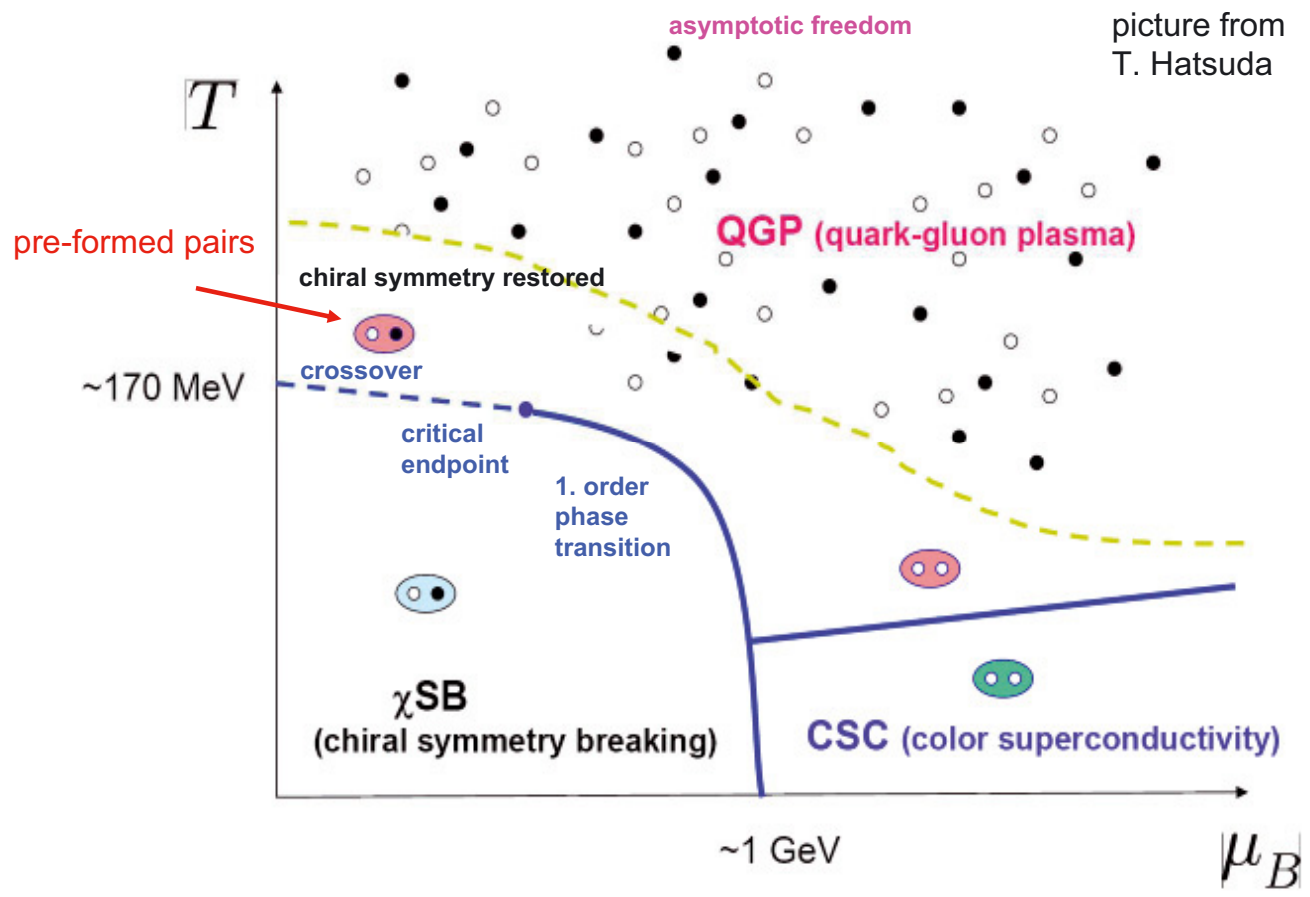

\section{The QCD Phase diagram: facts and speculations}

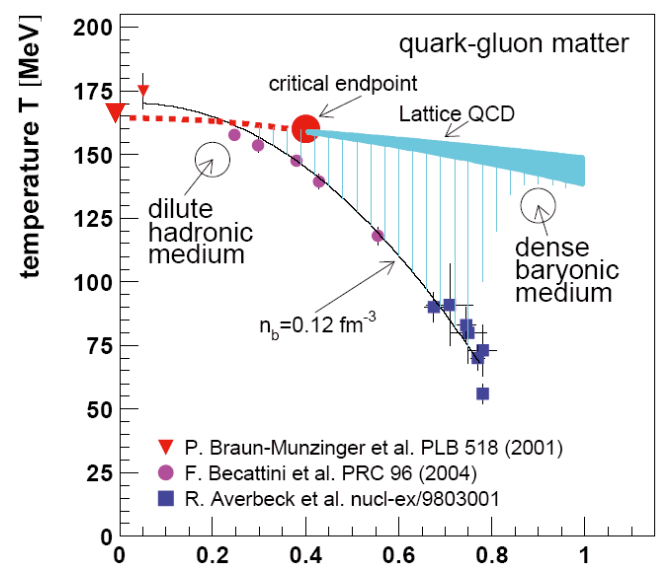

Experimental results:

$>$ Freeze-out curve $\left(T, \mu_{B}\right)$

$>T_{f o}=161 \pm 4 \mathrm{MeV}$ at $\left(\mu_{B}=0\right)$

$>$ new state of matter $=$ perfect liquid?

L-QCD Predictions:

$>\mathrm{T}_{C}=151 \pm 7 \pm 4 \mathrm{MeV}$

(Z. Fodor, arXiv:0712.2930 hep-lat)

$>\mathrm{T}_{C}=192 \pm 7 \pm 4 \mathrm{MeV}$

(F. Karsch, arXiv:0711.0661 hep-lat)

$>$ crossover transition at $\mu_{B}=0$

(Z. Fodor, arXiv:0712.2930 hep-lat)

$>1$. order phase transition

with critical endpoint at $\mu_{B}>0$

High-energy heavy-ion collision experiments:

RHIC, LHC: cross over transition, QGP at high $T$ and low $\rho$ Low-energy RHIC: search for QCD-CP with bulk observables NA61@SPS: search for QCD-CP with bulk observables MPD@NICA: search for the QCD mixed phase with bulk observables CBM@FAIR: scan of the phase diagram with bulk and rare observables 


\section{Trajectories from Transport models}

UrQMD: L.V. Bravina et al., Phys. Rev. C60 (1999) 044905

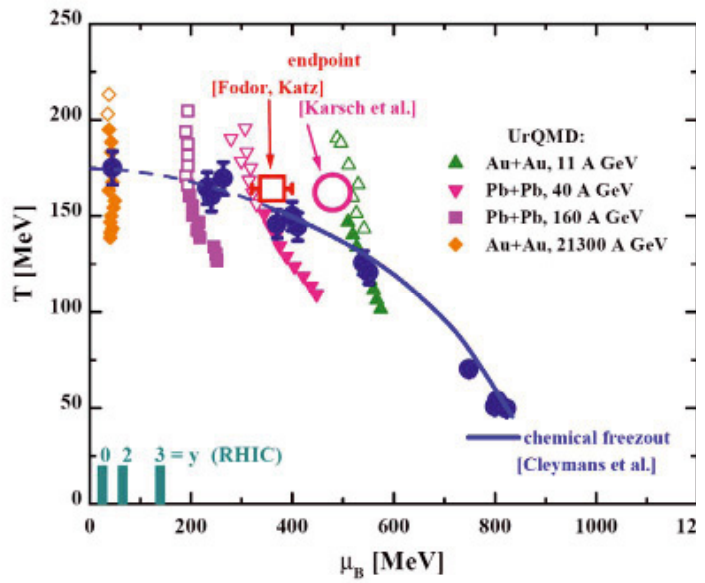

FAIR beam energies:

$A+A$ collisions up to 45 (35) $A G e V, Z / A=0.5(0.4)$

$(p+p$ and $p+A$ collisions up to $90 \mathrm{GeV}$ ) 3-fluid hydro:

Y. Ivanov, V. Russkikh, V.Toneev, Phys. Rev. C73 (2006) 044904

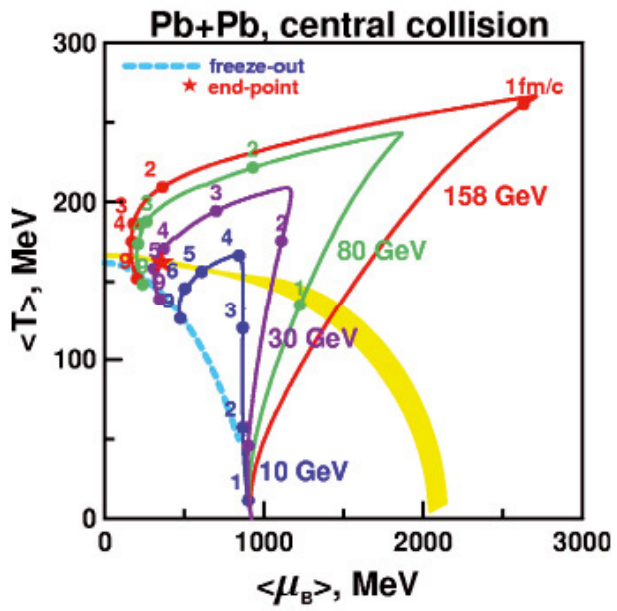




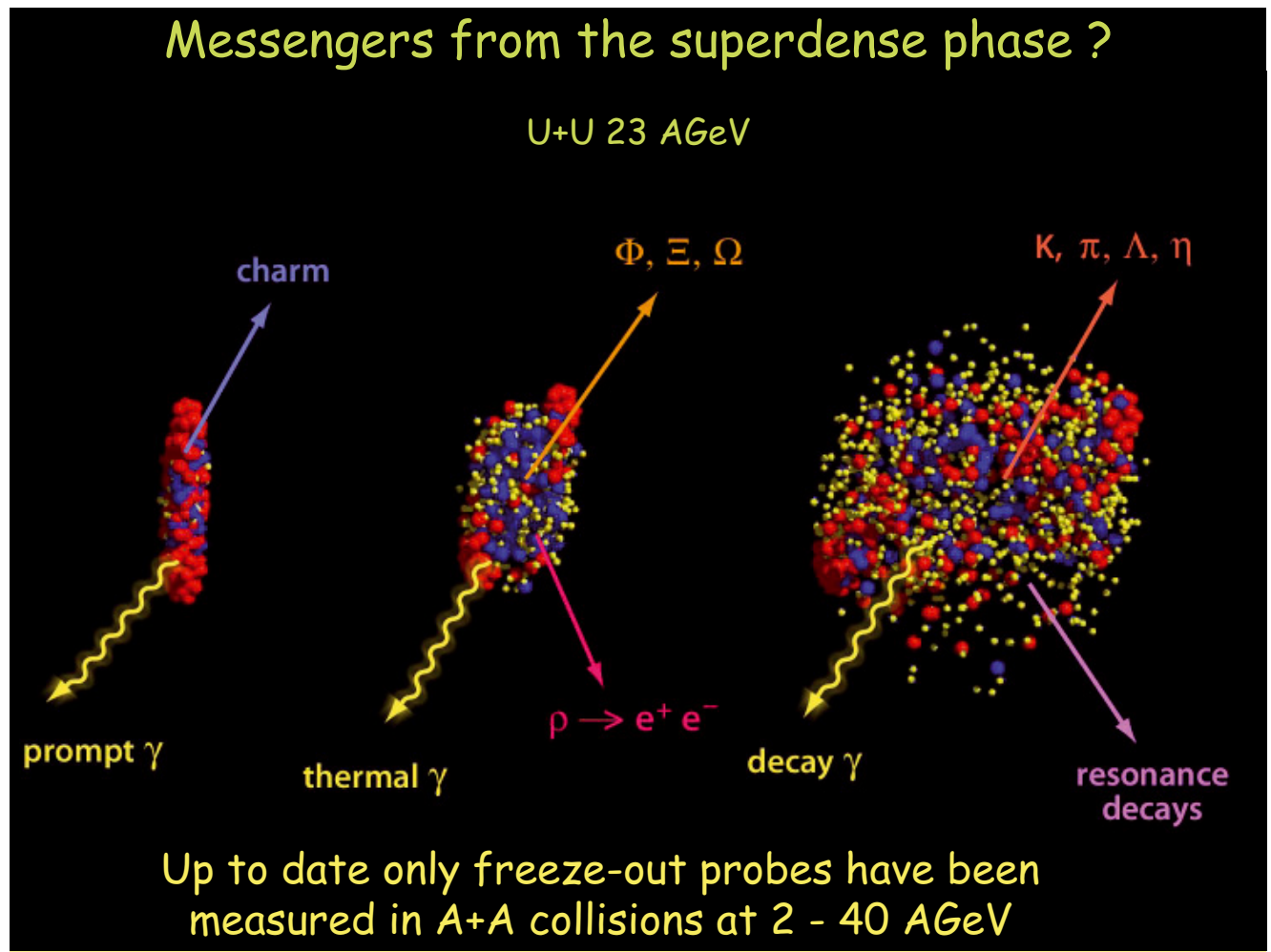

\section{Possible signatures of QGP in heavy-ion collisions}

taken from the book: Quark-Gluon-Plasma: from big bang to little bang by

Kohsuke Yagi, Tetsuo Hatsuda, Yasuo Miake

(2006)

adapted from an original by Shoji Nagamiya

\section{Seach for discontinuities} in excitation functions of various observables!

How much are the signals diluted by finite size, short lifetimes, and hadronization?

\section{transverse}

momentum

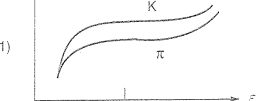

volume volume

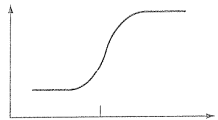

$\mathrm{N}_{\text {charm, }}$ chnarm, strangeness
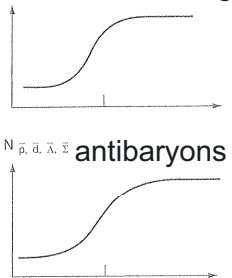

elliptic flow

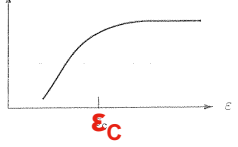

Energy density
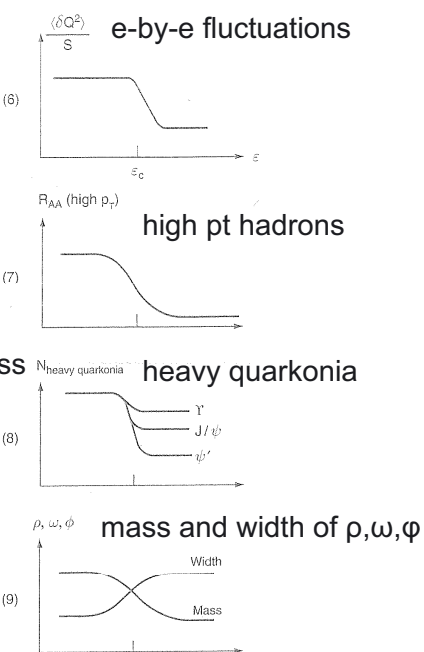

thermal photons and dileptons

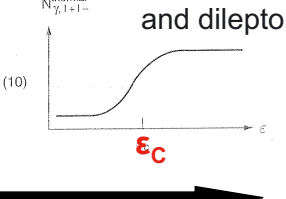




\section{Discontinuity in strangeness production: \\ signature for phase transition?}

C. Blume et al. (NA49 at CERN-SPS), nucl-ex/0409008
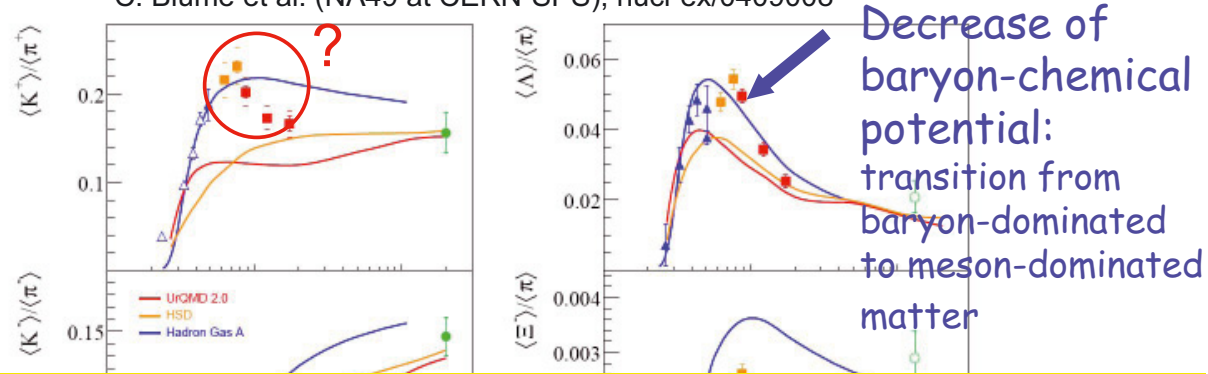

Low energy run at RHIC: remeasurement of $K / \pi$ (incl. e-by-e) CBM: Precise excitation function of multistrange particle production and propagation (collective flow)
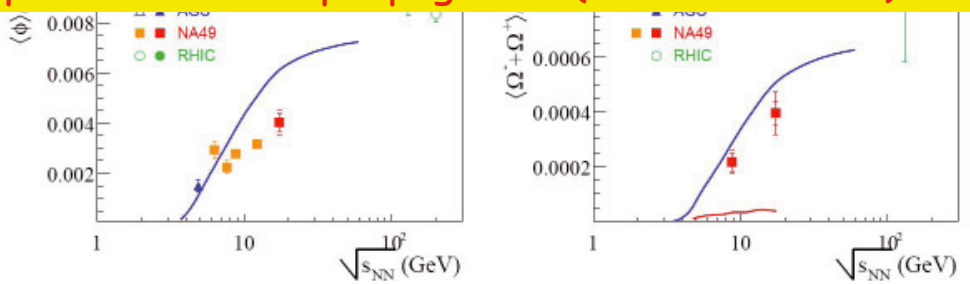

\section{Hadron elliptic flow at FAIR}

AMPT calculations: C.M. Ko at CPOD 2007

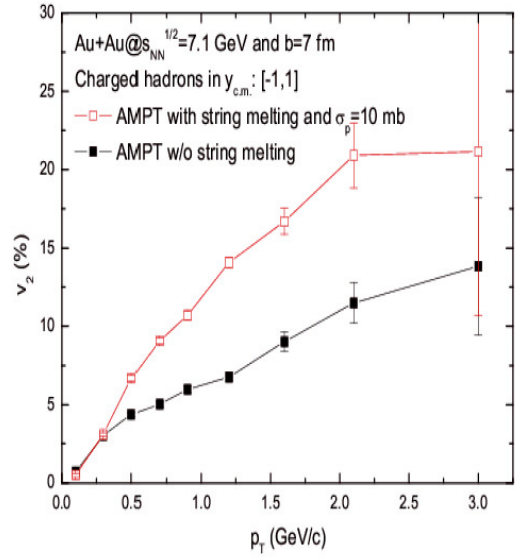

Partonic scattering enhances elliptic flow

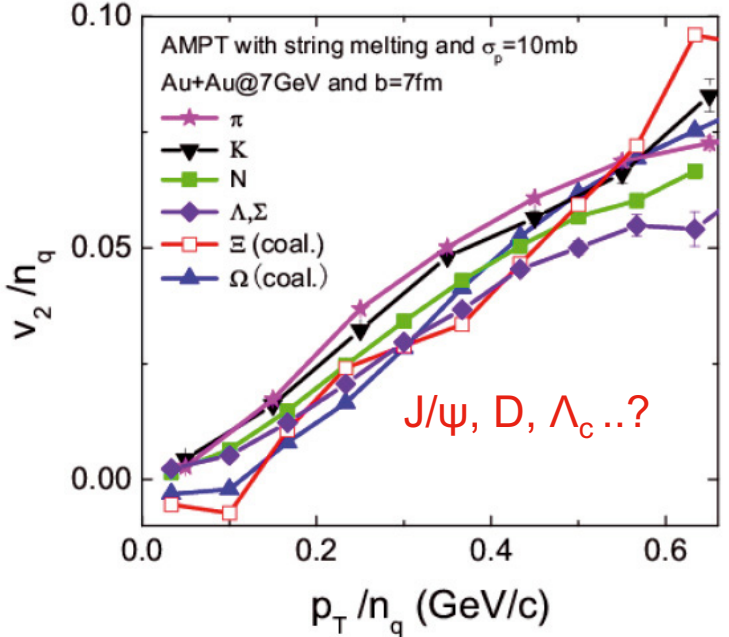

Approximate constituent quark number scaling ! 


\section{Charmonium dissociation in the quark-pluon plasma?}
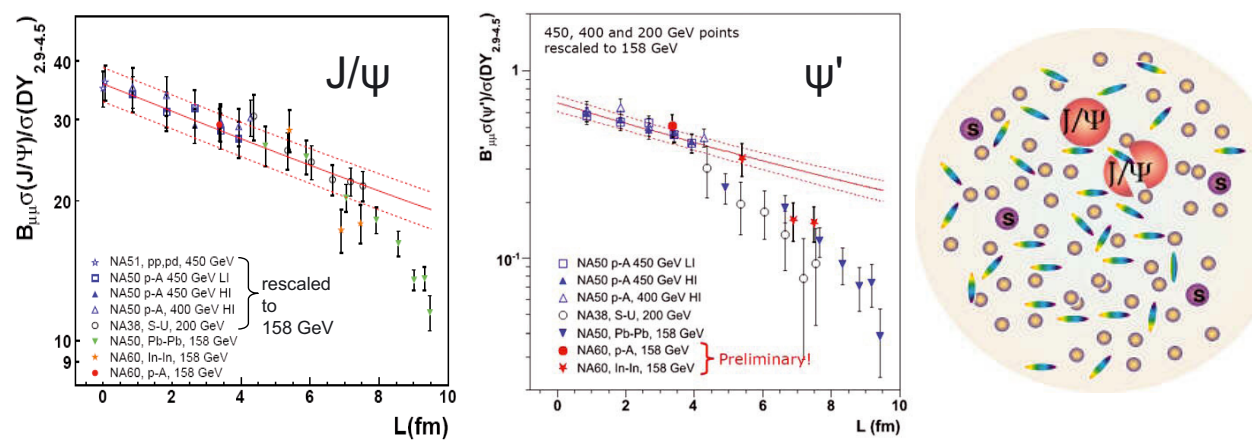

Quarkonium dissociation temperatures: (Digal, Karsch, Satz)

\begin{tabular}{|c||c|c|c||c|c|c|c|c|}
\hline state & $\mathrm{J} / \psi(1 S)$ & $\chi_{c}(1 \mathrm{P})$ & $\psi^{\prime}(2 S)$ & $\Upsilon(1 S)$ & $\chi_{b}(1 P)$ & $\Upsilon(2 S)$ & $\chi_{b}(2 P)$ & $\Upsilon(3 S)$ \\
\hline \hline$T_{d} / T_{c}$ & 2.10 & 1.16 & 1.12 & $>4.0$ & 1.76 & 1.60 & 1.19 & 1.17 \\
\hline
\end{tabular}

Upper bounds on dissociation temperatures: A.Mocsy, P. Petreczky

\begin{tabular}{||ccccccc||}
\hline \hline state & $\chi_{c}$ & $\psi^{\prime}$ & $J / \psi$ & $\Upsilon^{\prime}$ & $\chi_{b}$ & $\Upsilon$ \\
\hline$T_{\text {dis }}$ & $\leq T_{c}$ & $\leq T_{c}$ & $1.2 T_{c}$ & $1.2 T_{c}$ & $1.3 T_{c}$ & $2 T_{c}$ \\
\hline
\end{tabular}

sequential dissociation of $\mathrm{J} / \psi$ and $\psi^{\prime}$ ? measure excitation functions of $J / \psi, \psi^{\prime}, D, \Lambda_{C}$ production in $p+p, p+A$ and $A+A$ collisions (incl. $p_{T}$-spectra, flow)

\section{Charm production}

Hadronic transport models: parameterization of measured cross sections

O. Linnyk, E. Bratkovskaya, W. Cassing, H. Stöcker, Nucl. Phys. A786 (2007) 183
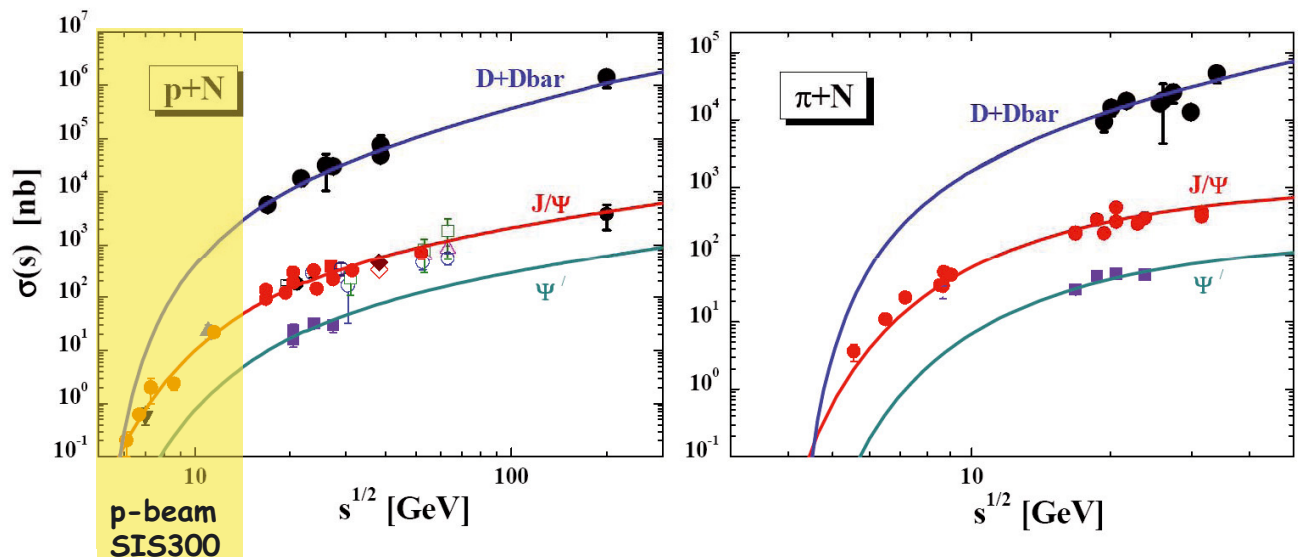


\section{The creation of charm} in hadronic transport calculations

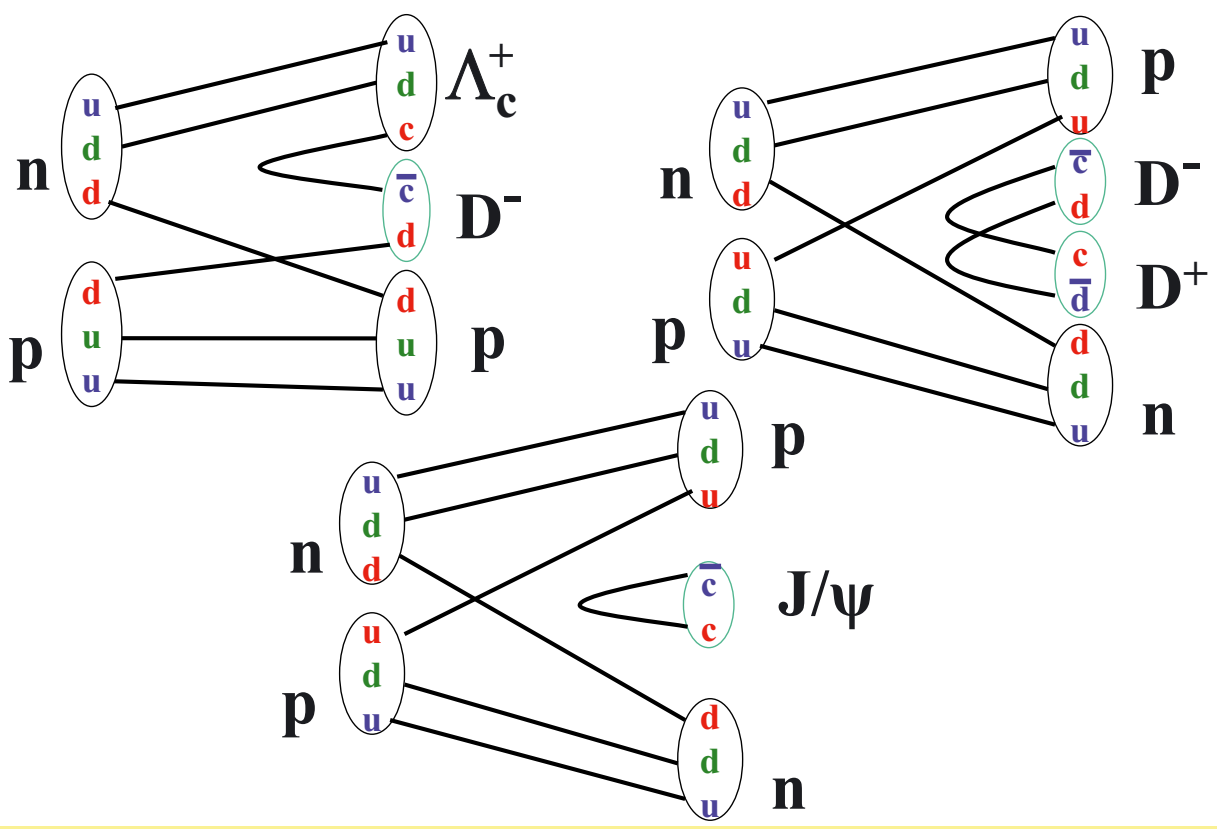

The creation of charm via hard collisions (gg and 99 ) in a partonic phase

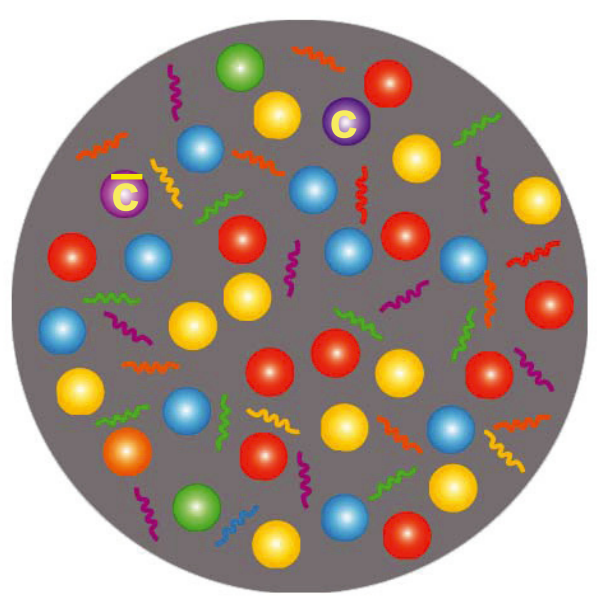

Formation of $D$ mesons, charmed hyperons and charmonium via coalescence (e.g. statistical hadronization) 


\section{Charmonium and open charm production by statistical hadronisation in nuclear collisions}

A. Andronic, P. Braun-Munzinger, K. Redlich, J. Stachel, arXiv:0708.1488

1. c-cbar production in hard collisions,

2. statistical hadronization according to the temperature and the baryochemical potential
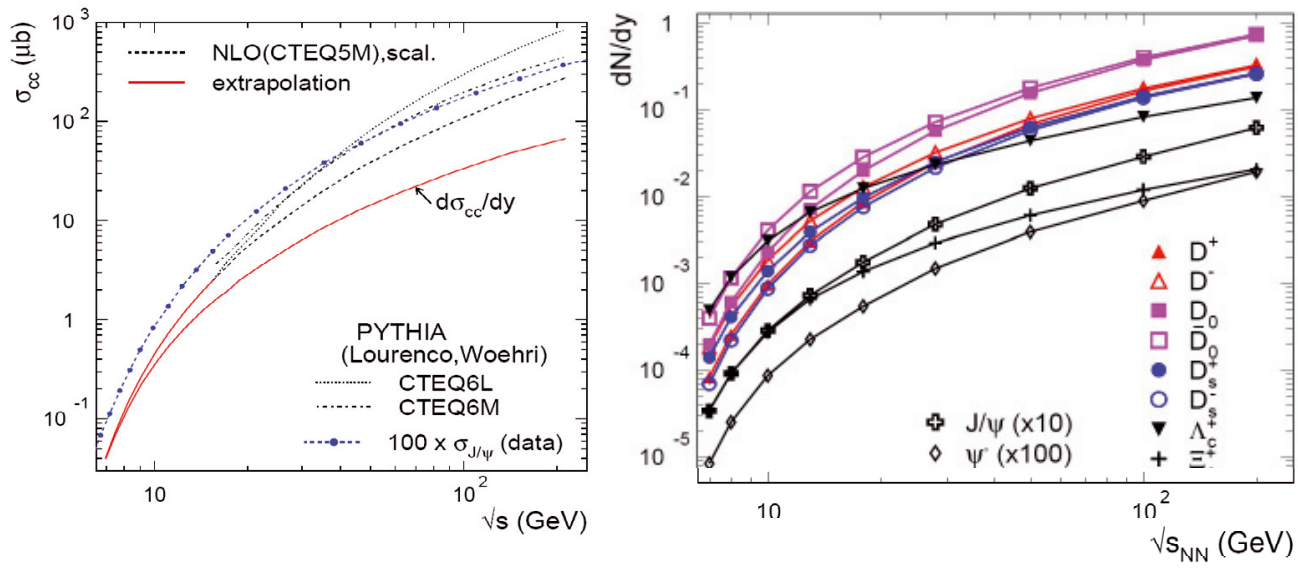

Charm production in partonic and hadronic matter

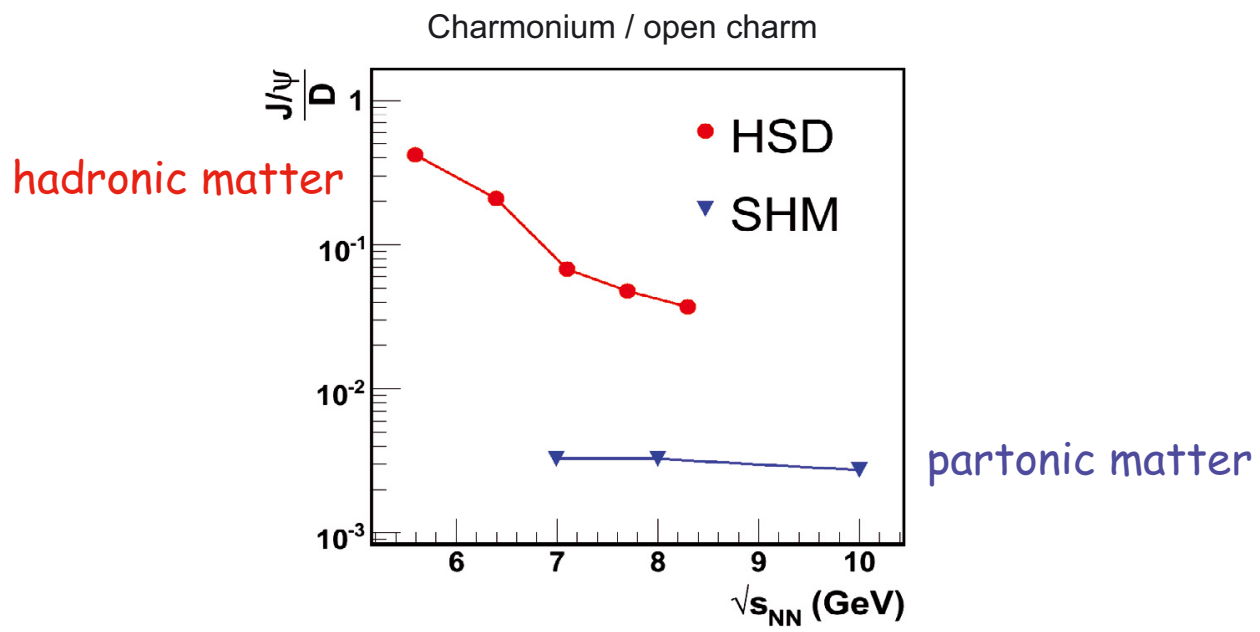

Charmed particle ratios $\left(\psi / D, \Lambda_{c} / D, D / D_{s}, \ldots\right)$ are sensitive to the medium they are formed in 


\section{In-medium properties of open charm}
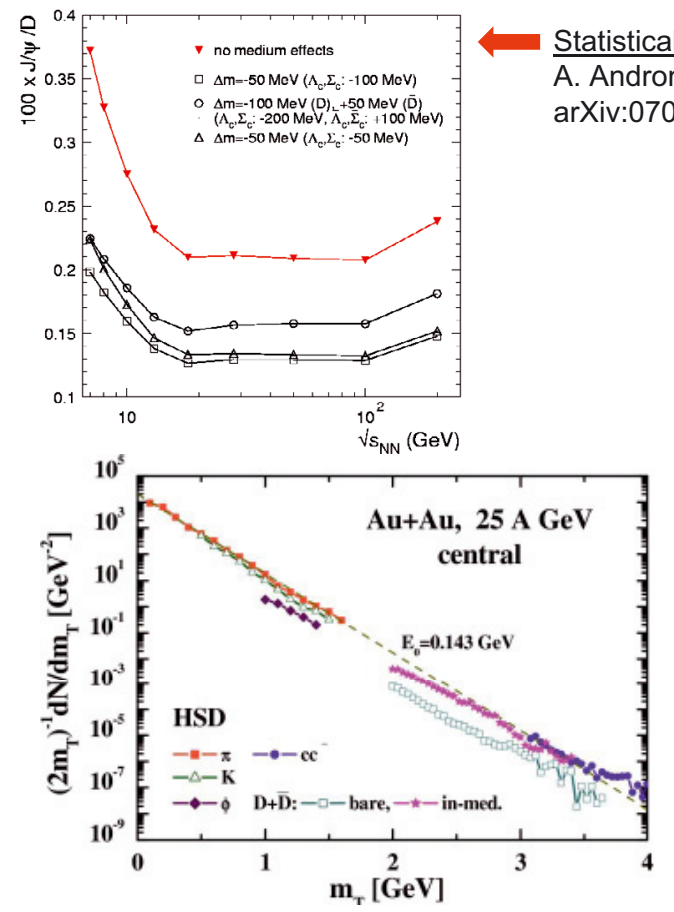

Statistical hadronization model:

A. Andronic, P. Braun-Munzinger, K. Redlich, J. Stachel, arXiv:0708.1488

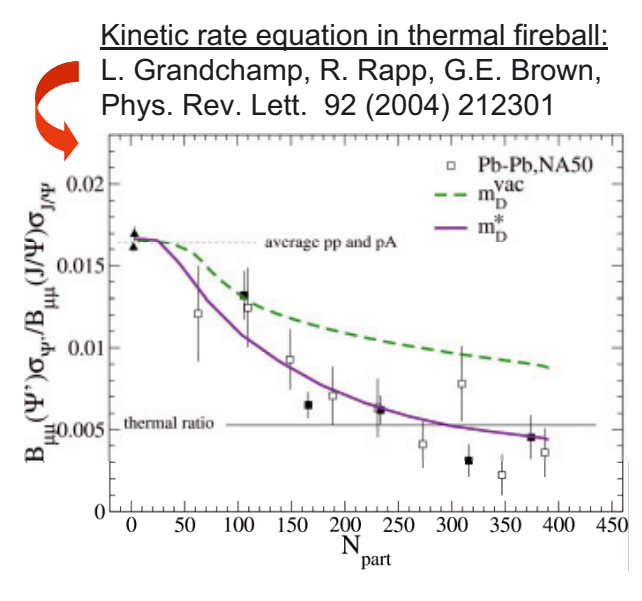

HSD microscopic transport model:

W. Cassing, E. Bratkovskaya, A. Sibirtsev, Nucl. Phys. A 691 (2001) 753

Looking into the fireball ...

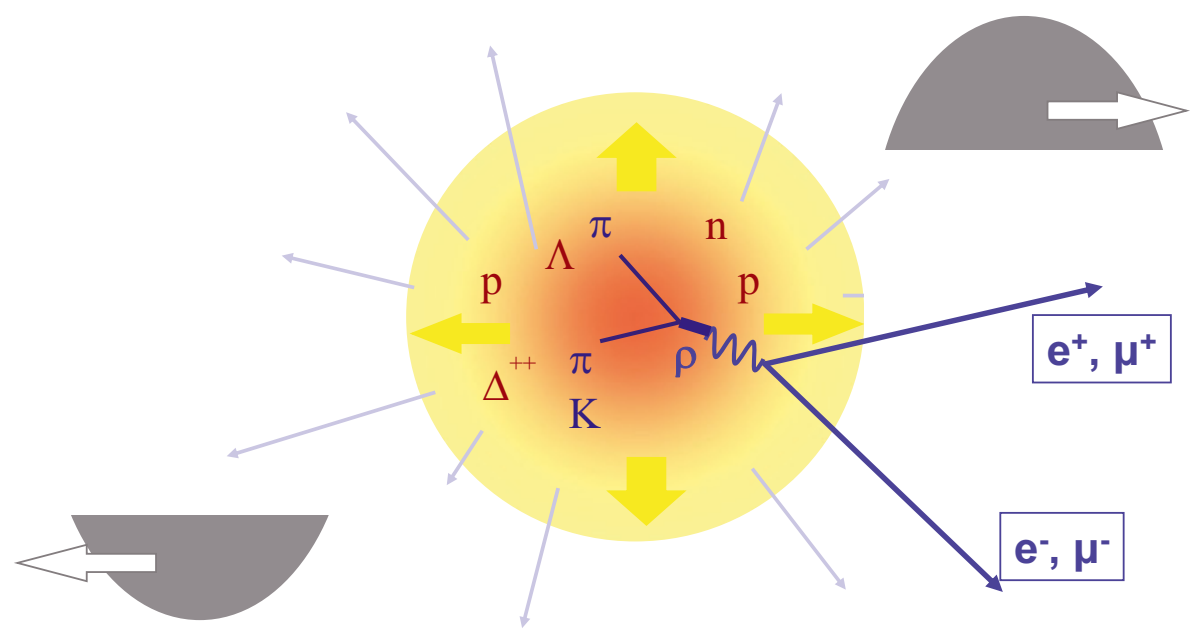

... using penetrating probes:

short-lived vector mesons decaying into electron-positron pairs 


\section{Dilepton radiation from the deconfined phase?}

Data: R. Arnaldi, et al. [NA60 Collaboration], Phys. Rev.Lett. 96, 162302 (2006);

Calculation: J.Ruppert, C. Gale, Th. Renk, P. Lichard, J. I. Kapusta, arXiv:0706.1934

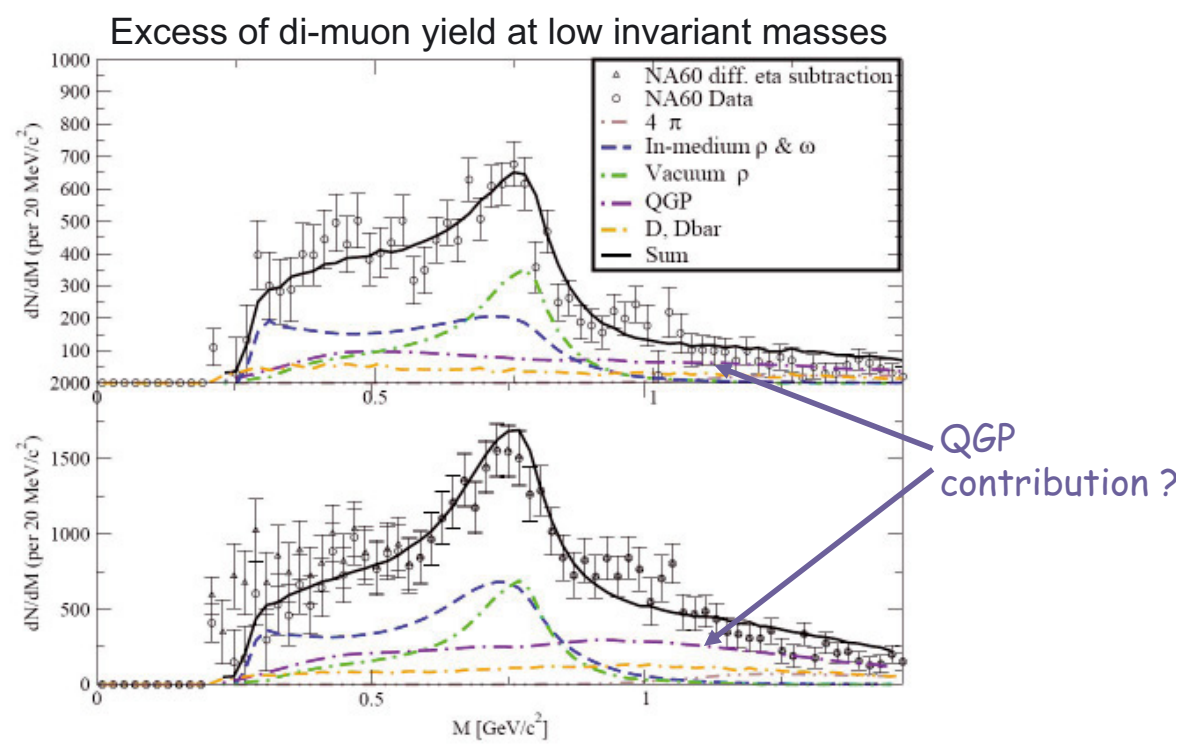

\section{Inverse slope parameters as function of mass}

R. Arnaldi et al., (NA60), arXiv: 0711.1816v1 [nucl-ex] to appear in PRL

N. Xu, Int. J. Mod. Phys. E16 (2007) 715
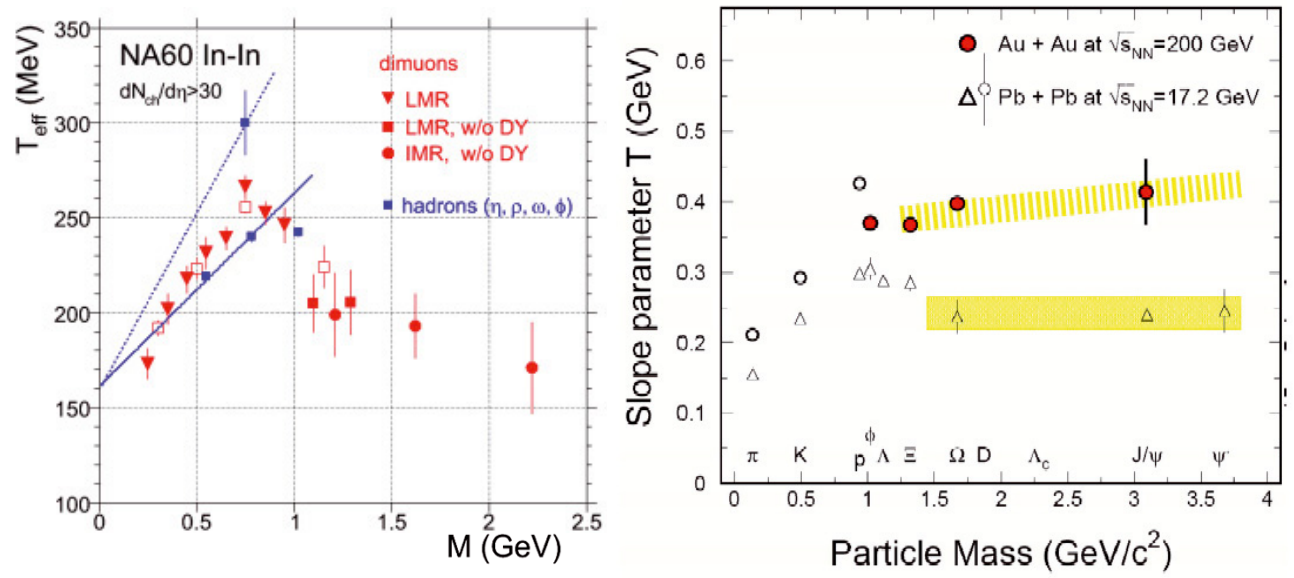

$M<1 \mathrm{GeV} / \mathrm{c}^{2}$ : radial flow generated in the late hadronic phase $M>1 \mathrm{GeV} / \mathrm{c}^{2}$ : messengers from the early partonic phase? 


\section{Searching for the modification of hadron properties via dilepton measurements}

Calculations: H. van Hees, R. Rapp, arXiv:0711.3444v1 [hep-ph]
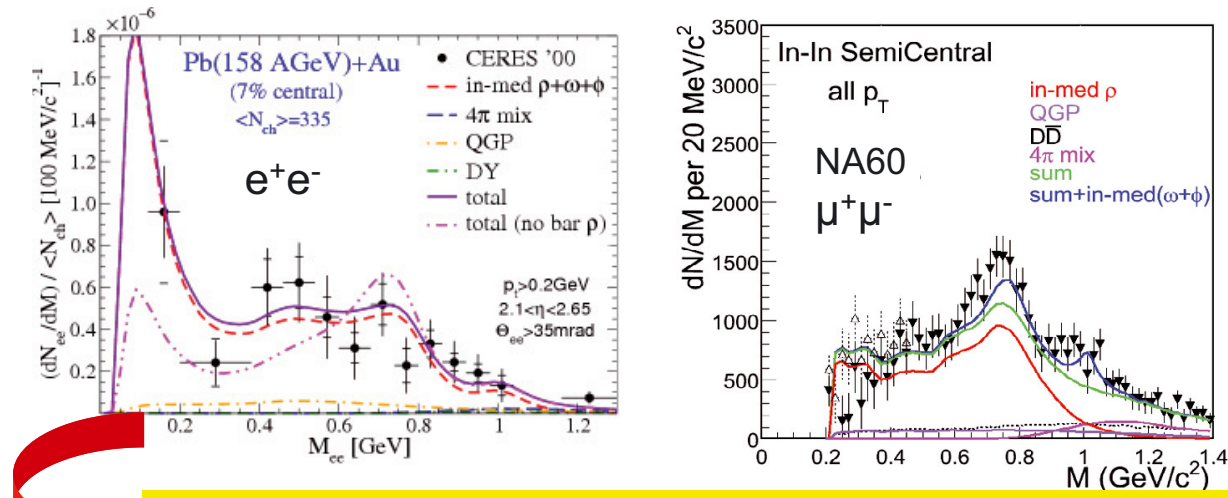

no $\rho, \omega, \varphi \rightarrow e^{+} e^{-}\left(\mu^{+} \mu^{-}\right)$data between 2 and $40 \mathrm{AGeV}$

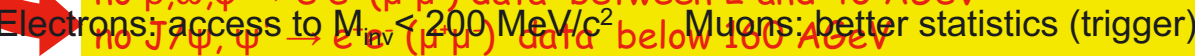

Information needed in addition to dileptons from $A+A$ :

$\pi, K, p, \Lambda, \ldots$ to constrain the fireball evolution

$>\rho \rightarrow \pi \pi, \varphi \rightarrow K K$ to measure the freeze-out contributions

$>p+A$ collisions to determine the contribution of primordial $\rho$ 's

The quest for the critical endpoint:

search for dynamical fluctuations in the E-by- $E \mathrm{kaon} /$ pion ratio
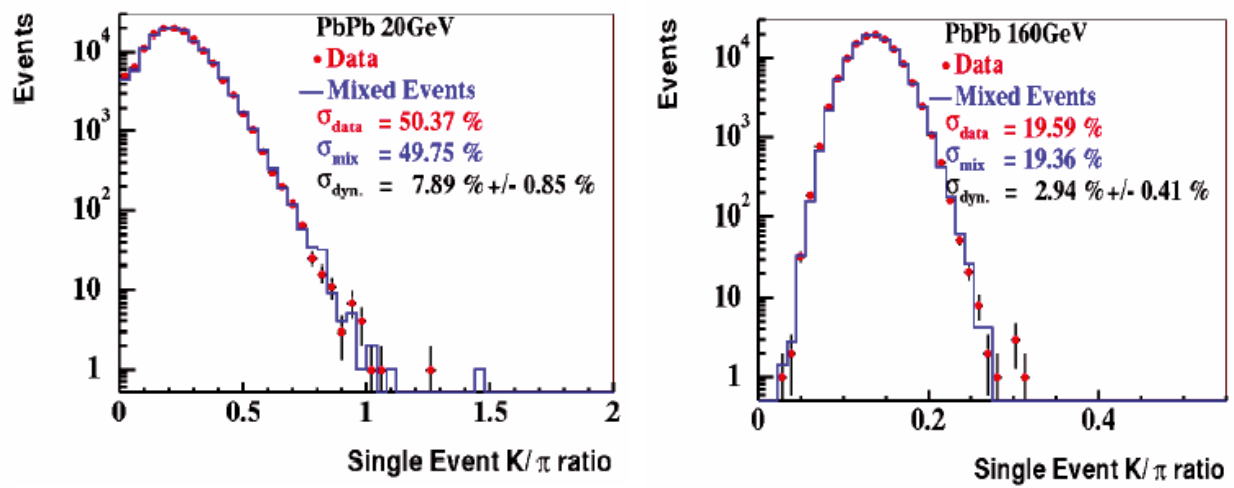

NA49 Preliminary 
Measure the particle yields ratio in an event :

\author{
$\mathrm{K} / \pi$
}

Relative width of distribution : $\sigma=\mathrm{RMS} / \mathrm{MEAN}$

$$
\sigma_{\text {data }}^{2}=\underbrace{\sigma_{\text {fin }}^{2}+\sigma_{\text {exp }}^{2}+\sigma_{\text {dyn }}^{2}}_{\text {background }}
$$

Event mixing: no two tracks coming from one real event

$$
\sigma_{\text {dyn }}^{2}=\sigma_{\text {data }}^{2}-\sigma_{\text {mixed }}{ }^{2}
$$

\title{
Fluctuations from NA49
}

nucl-ex/0403035
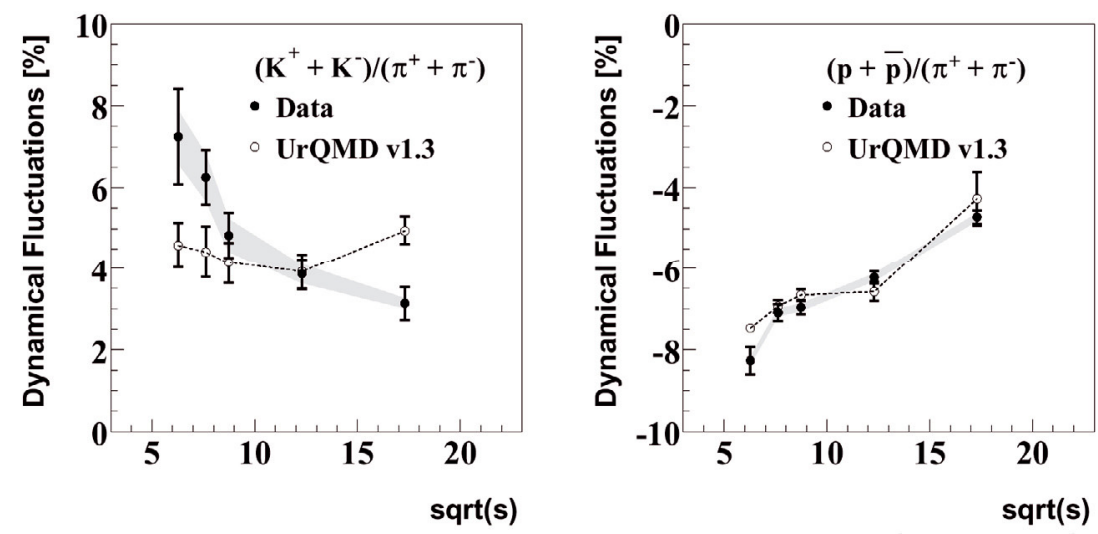

- dynamical fluctuations reported by NA49

- increase towards low energies

- $\mathrm{K} / \pi$ : not reproduced by UrQMD

- $p / \pi$ : correlation due to resonance decays 


\section{Summary: CBM physics topics and observables}

The equation-of-state at high $\rho_{B}$

$>$ collective flow of hadrons

$>$ particle production at threshold energies

(multistrange hyperons, open charm?)

Deconfinement phase transition at high $\rho_{B}$

$>$ excitation function and flow of strangeness $(K, \Lambda, \Sigma, \Xi, \Omega)$

$>$ excitation function and flow of charm $\left(J / \psi, \psi^{\prime}, D^{0}, D^{ \pm}, \Lambda_{c}\right)$

(e.g. melting of $J / \psi$ and $\psi^{\prime}$ )

$>$ excitation function of low-mass lepton pairs

QCD critical endpoint

$>$ excitation function of event-by-event fluctuations $(K / \pi, \ldots)$

Onset of chiral symmetry restoration at high $\rho_{B}$

$>$ in-medium modifications of hadrons $\left(\rho, \omega, \phi \rightarrow e^{+} e^{-}\left(\mu^{+} \mu^{-}\right), D\right)$

\section{CBM Physics Book in preparation}

\section{Meson production in central $\mathrm{Au}+\mathrm{Au}$ collisions}

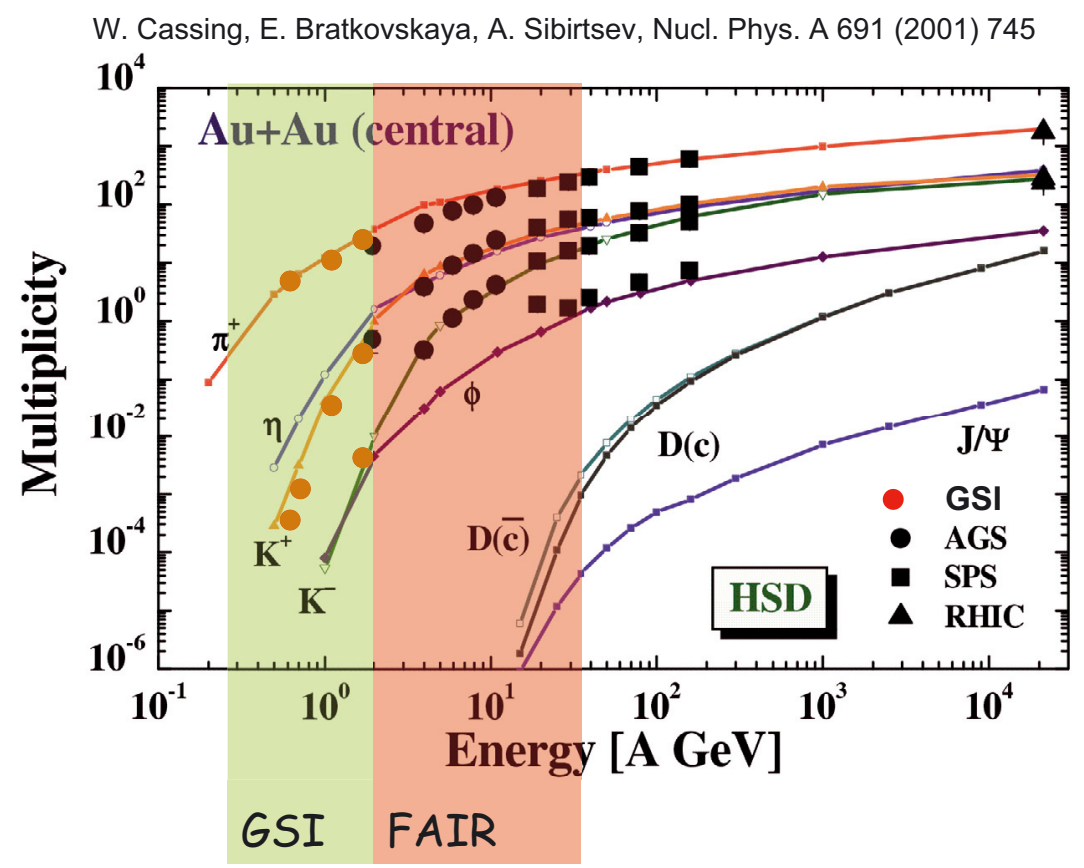




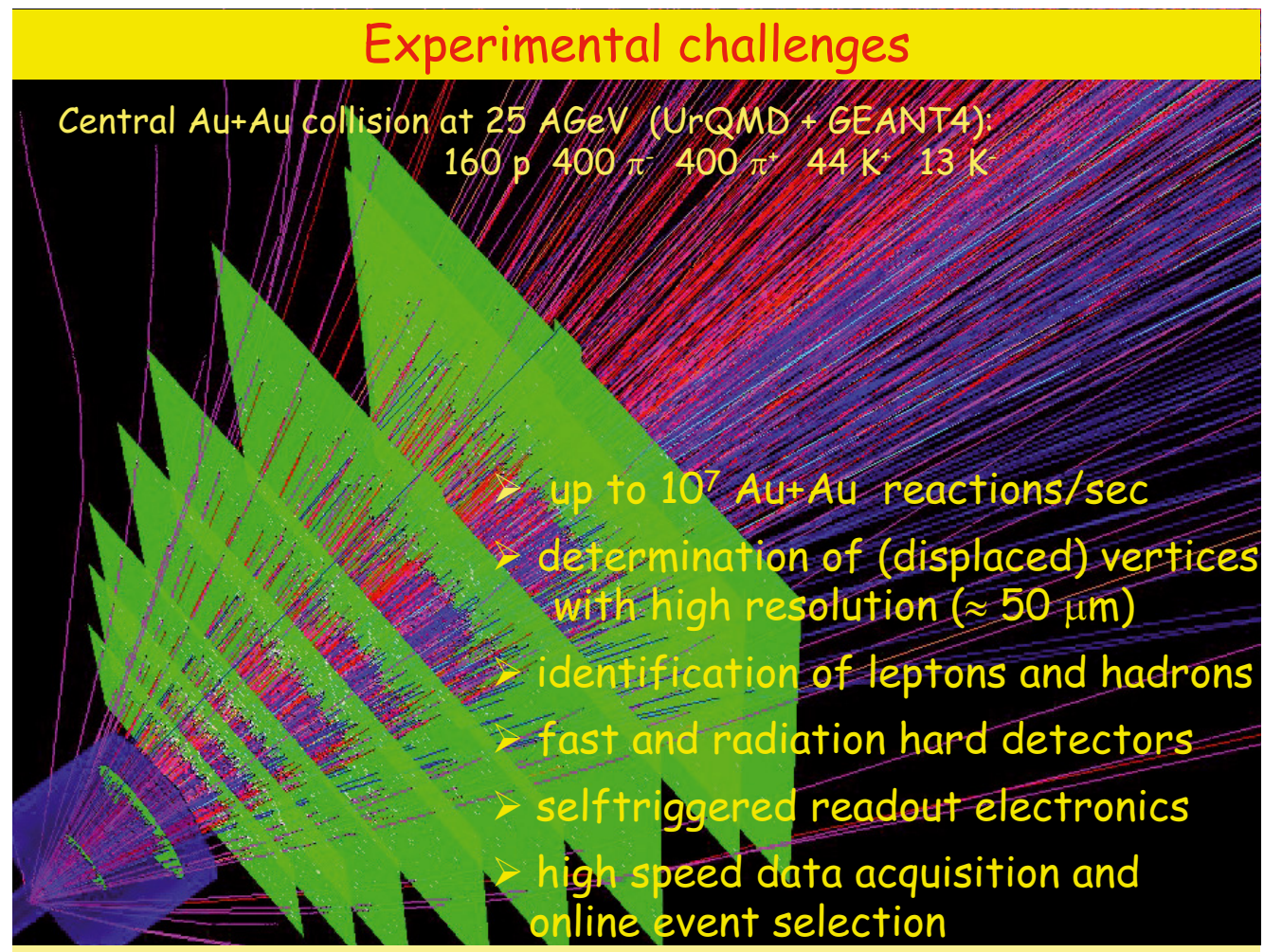

The Compressed Baryonic Matter Experiment

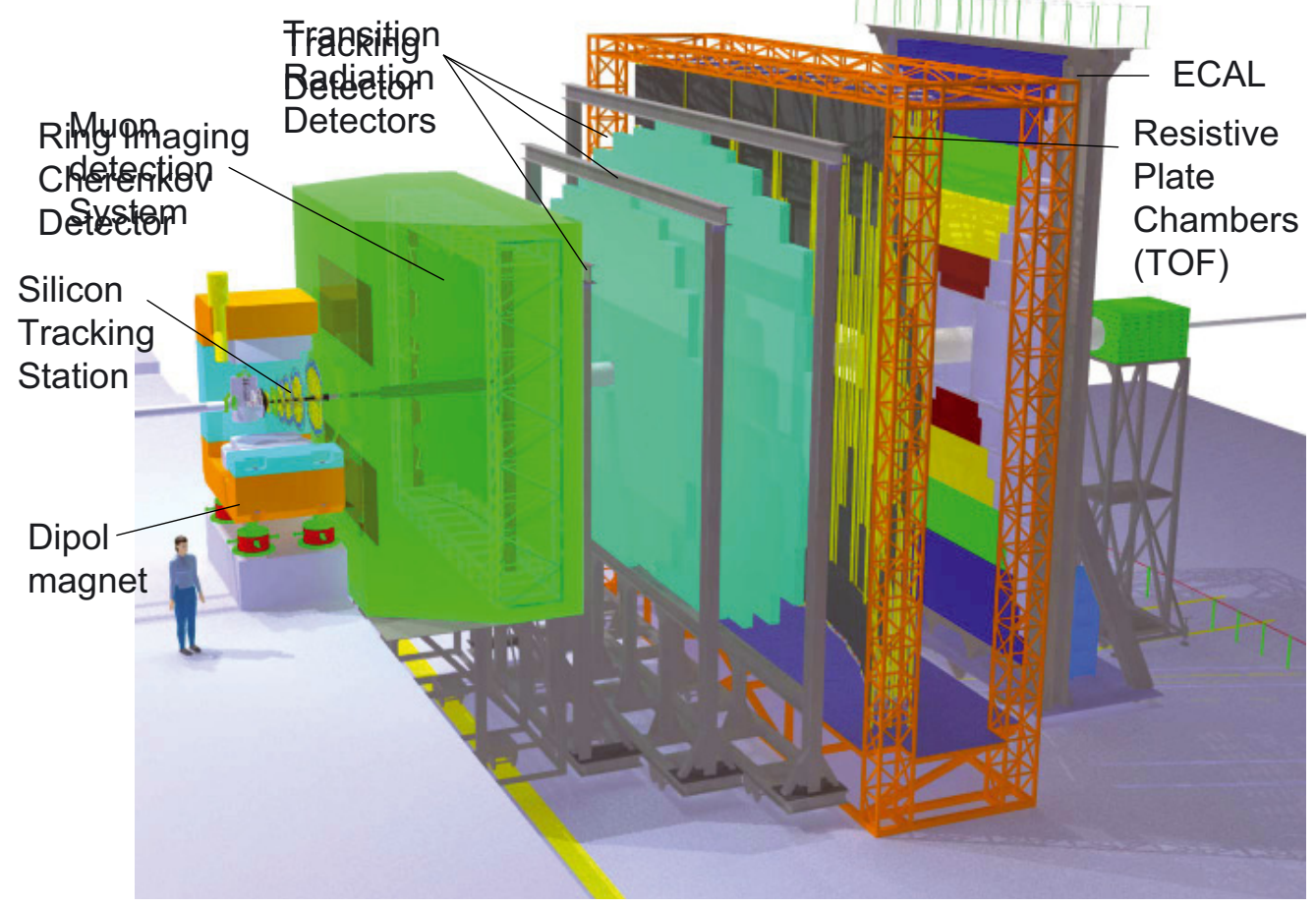




\section{Conceptual design and feasibility studies}

Software tools:

- Framework FAIRroot, VMC

- Transport codes GEANT3\&4, FLUKA

- Event generators URQMD, HSD, PLUTO

- Fast track reconstruction algorithms for online event selection Simulation input:

- realistic signal and background multiplicities (e.g. central coll. Au+Au $25 \mathrm{AGeV}$ )

- realistic detector layout and response
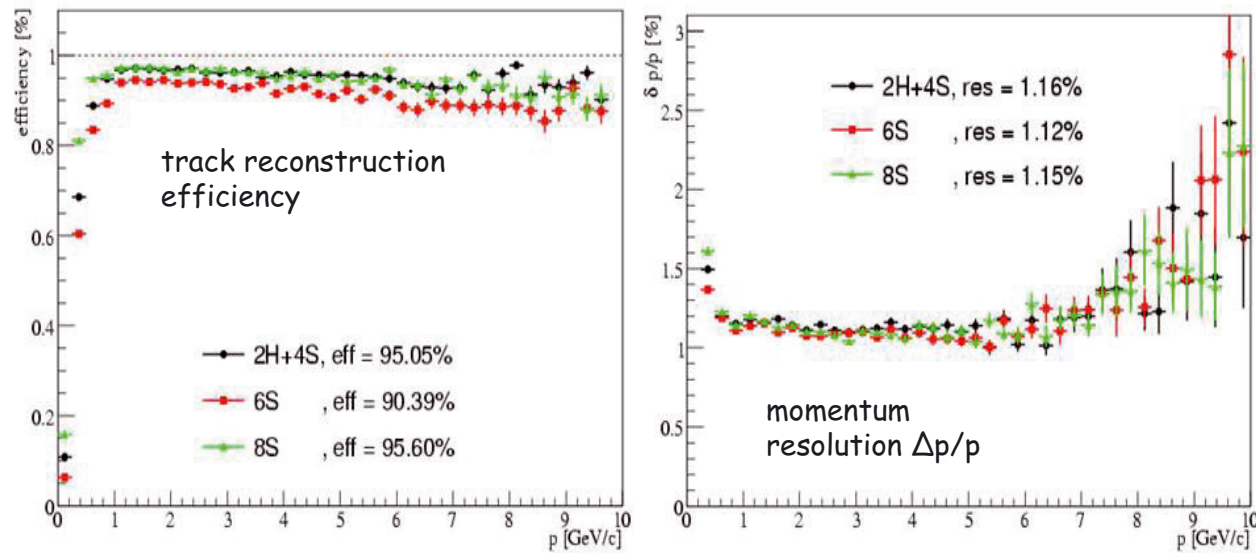

\section{Hyperon detection with STS (no p, K, $\pi$ identification)}

Silicon tracker: 2 hybrid pixel (750 $\mu$ m each), 4 microstrips (400 $\mu \mathrm{m}$ each)

$>$ Strips with $50 \mu \mathrm{m}$ pitch and $5^{\circ}$ stereo angle

$>$ full event reconstruction

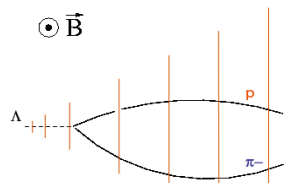

central Au+Au collisions at 25 AGeV:

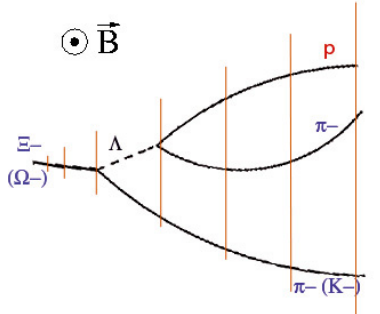

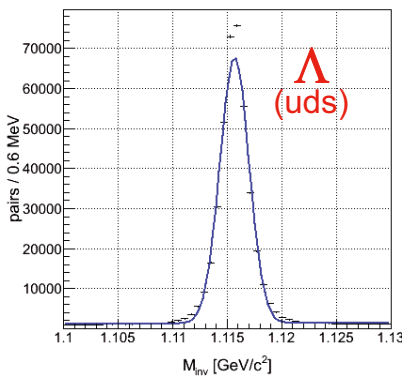

total efficiency $10.6 \%$

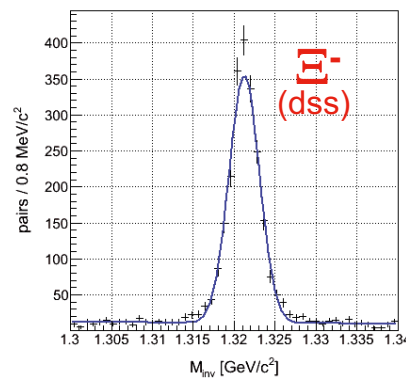

$2.1 \%$

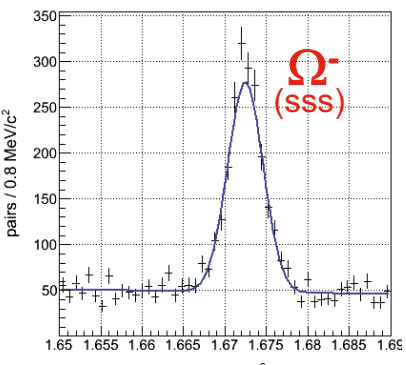

$1.0 \%$ 


\section{Benchmark for Vertex-Tracker performance: \\ $D$ mesons and $\Lambda_{C}$ from $\mathrm{Au}+\mathrm{Au}$ central collisions at $25 \mathrm{AGeV}$}

Full track reconstruction:

- 2 MAPS (150 um thickness each)

- 6 Silicon micro-strip detectors

- proton identification via TOF

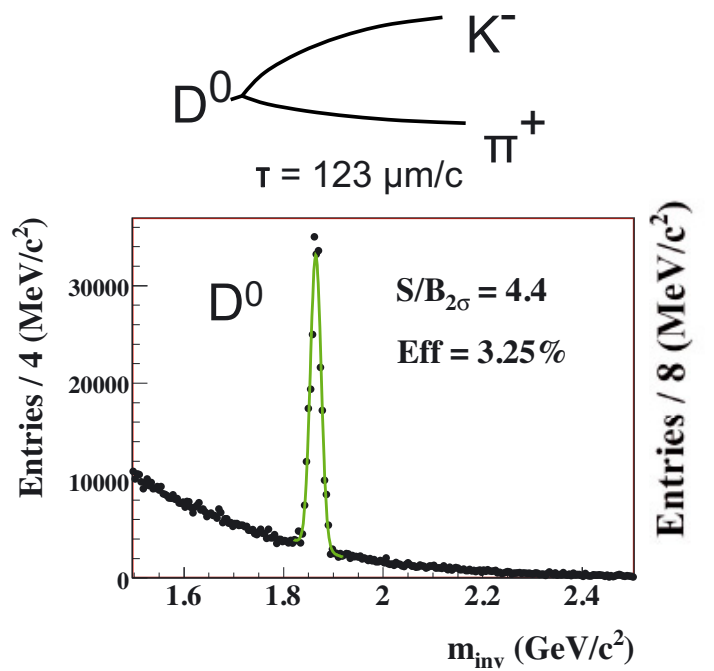

$D$ and $\Lambda_{C}$ multiplicity from HSD

Hadronic background from UrQMD

Vector meson identification via electron pairs in CBM
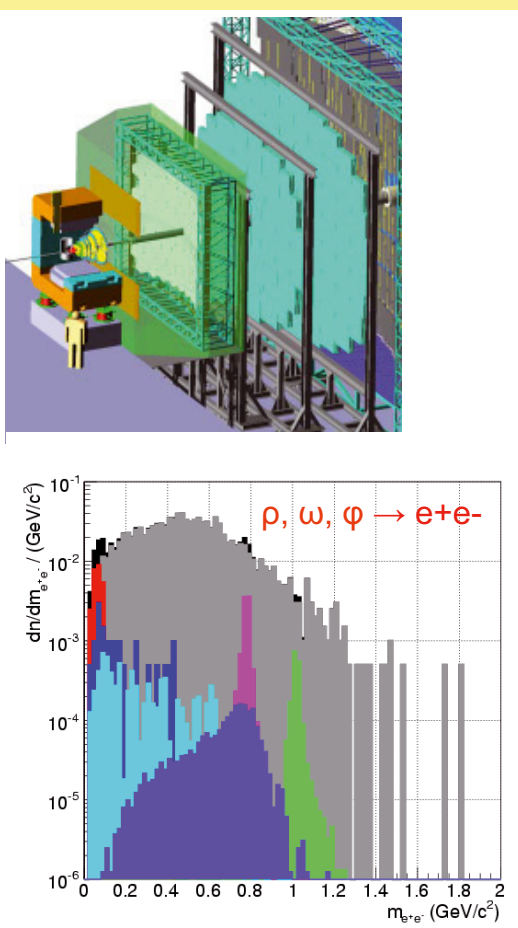

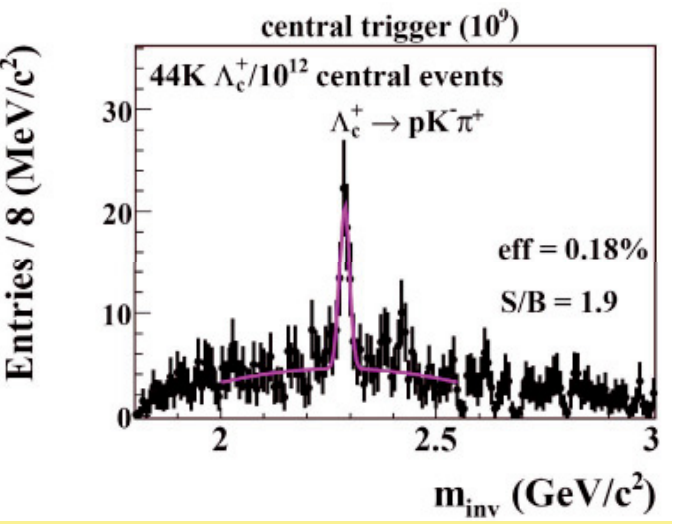

$\Lambda_{\mathrm{C}} \rightarrow \pi^{+} \mathrm{K}-\mathrm{p}$

$\mathrm{T}=60 \mu \mathrm{m} / \mathrm{c}$

central trigger $\left(10^{9}\right)$

$\mathrm{m}_{\text {inv }}\left(\mathrm{GeV} / \mathrm{c}^{2}\right)$

central $\mathrm{Au}+\mathrm{Au}$ collisions at $25 \mathrm{AGeV}$

\begin{tabular}{|c|c|c|c|}
\hline particle & $\mathrm{S} / \mathrm{B}$ & $\varepsilon(\%)$ & $\sigma(\mathrm{MeV})$ \\
\hline $\boldsymbol{\omega}$ & 0.15 & 7.5 & 14 \\
\hline $\boldsymbol{\varphi}$ & 0.13 & 9.1 & 14 \\
\hline $\boldsymbol{\rho}$ & 0.002 & 4 & \\
\hline $\mathbf{J} / \boldsymbol{\psi}$ & 10 & 12 & 38 \\
\hline $\boldsymbol{\Psi}^{\prime}$ & & & \\
\hline
\end{tabular}

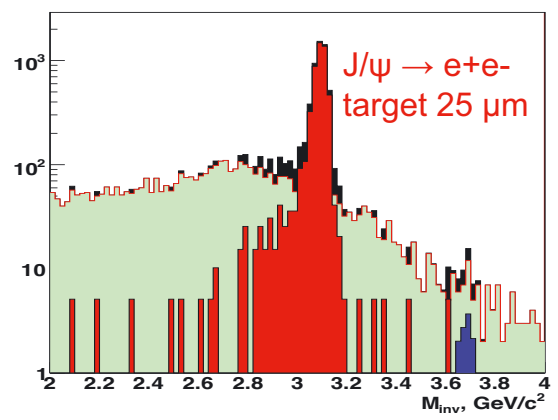




\section{Vector meson identification via muon pairs in CBM}

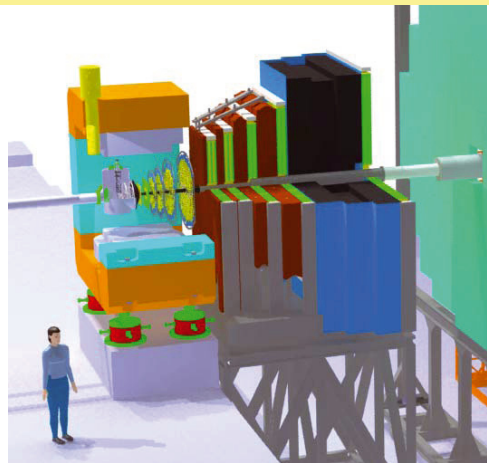

\section{Central Au+Au collisions at $25 \mathrm{AGeV}$}

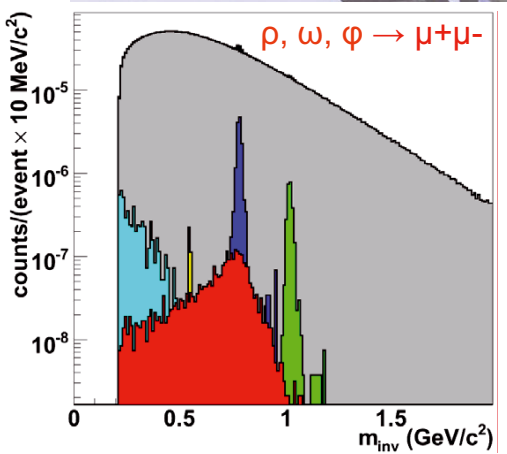

\begin{tabular}{|c|c|c|c|}
\hline particle & S/B & $\varepsilon(\%)$ & $\sigma(\mathrm{MeV})$ \\
\hline$\omega$ & 0.11 & 4 & 10 \\
\hline$\varphi$ & 0.06 & 7 & 12 \\
\hline$\rho$ & 0.002 & 3 & \\
\hline $\mathrm{J} / \psi$ & 18 & 13 & 21 \\
\hline$\Psi^{\prime}$ & 1 & 16 & 27 \\
\hline
\end{tabular}

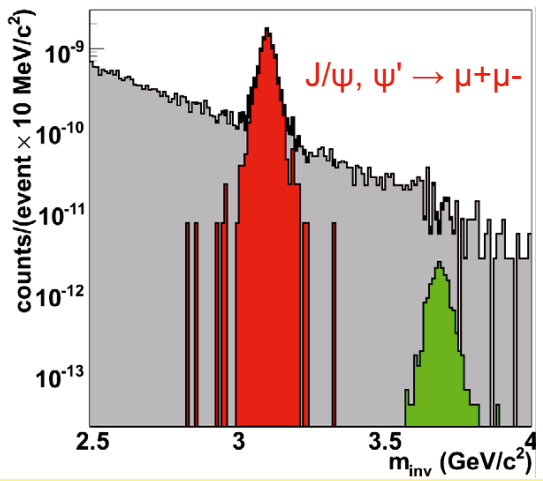

Estimated particle yields for minimum bias Au+Au collisions at $25 \mathrm{AGeV}$

\begin{tabular}{|c|c|c|c|c|c|c|c|c|}
\hline $\begin{array}{c}\text { particle, } \\
\text { mass }(\mathrm{MeV})\end{array}$ & $\mathrm{N}$ & $\begin{array}{l}\text { decay } \\
\text { mode }\end{array}$ & $\mathrm{BR}$ & $\begin{array}{c}\mathrm{R} / \mathrm{s} \\
(\mathrm{MHz})\end{array}$ & $\mathrm{T}$ & $\begin{array}{c}\varepsilon \\
(\%)\end{array}$ & $\mathrm{Y} / \mathrm{s}$ & $\mathrm{Y} / 10 \mathrm{w}$ \\
\hline$\eta(547)$ & 6.6 & $\mu^{+} \mu^{-}$ & $5.8 \cdot 10^{-6}$ & 0.25 & $\mathrm{y}$ & 3 & 0.28 & $1.7 \cdot 10^{6}$ \\
\hline$K^{+}(494)$ & 8 & - & - & 0.025 & $\mathrm{n}$ & 20 & $4 \cdot 10^{4}$ & $2.4 \cdot 10^{11}$ \\
\hline$K^{-}(494)$ & 2.6 & - & - & 0.025 & $\mathrm{n}$ & 20 & $1.3 \cdot 10^{4}$ & $7.8 \cdot 10^{10}$ \\
\hline$K_{s}^{0}(497)$ & 5.4 & $\pi^{+} \pi^{-}$ & 0.69 & 0.025 & $\mathrm{n}$ & 10 & $9.3 \cdot 10^{3}$ & $5.6 \cdot 10^{10}$ \\
\hline$\rho(770)$ & 4.6 & $e^{+} e^{-}$ & $4.7 \cdot 10^{-5}$ & 0.025 & $\mathrm{n}$ & 5.4 & 0.29 & $1.8 \cdot 10^{6}$ \\
\hline$\rho(770)$ & 4.6 & $\mu^{+} \mu^{-}$ & $4.6 \cdot 10^{-5}$ & 0.25 & $\mathrm{y}$ & 2.7 & 1.4 & $8.6 \cdot 10^{6}$ \\
\hline$\omega(782)$ & 7.6 & $e^{+} e^{-}$ & $7.1 \cdot 10^{-5}$ & 0.025 & $\mathrm{n}$ & 7.2 & 1 & $6 \cdot 10^{6}$ \\
\hline$\omega(782)$ & 7.6 & $\mu^{+} \mu^{-}$ & $9 \cdot 10^{-5}$ & 0.25 & $\mathrm{y}$ & 3.7 & 6.3 & $38 \cdot 10^{6}$ \\
\hline$\phi(1020)$ & 0.256 & $e^{+} e^{-}$ & $3 \cdot 10^{-4}$ & 0.025 & $\mathrm{n}$ & 9.6 & 0.18 & $1 \cdot 10^{6}$ \\
\hline$\phi(1020)$ & 0.256 & $\mu^{+} \mu^{-}$ & $2.9 \cdot 10^{-4}$ & 0.25 & $\mathrm{y}$ & 6 & 1. & $6.7 \cdot 10^{6}$ \\
\hline$\Lambda(1115)$ & 6.4 & $\mathrm{p} \pi^{-}$ & 0.64 & 0.025 & $\mathrm{n}$ & 10.6 & $1.1 \cdot 10^{4}$ & $6.5 \cdot 10^{10}$ \\
\hline$\Xi^{-}(1321)$ & 0.096 & $\Lambda \pi^{-}$ & 0.999 & 0.025 & $\mathrm{n}$ & 2.1 & 50.4 & $3 \cdot 10^{8}$ \\
\hline$\Omega^{-}(1672)$ & 0.0044 & $\bar{\Lambda} \Lambda K^{-}$ & 0.68 & 0.025 & $\mathrm{n}$ & 1 & 0.75 & $4.5 \cdot 10^{6}$ \\
\hline$D^{0}(1864)$ & $7.5 \cdot 10^{-6}$ & $K^{-} \pi^{+}$ & 0.038 & 0.1 & $\mathrm{y}$ & 3.25 & $8.5 \cdot 10^{-4}$ & $5.1 \cdot 10^{3}$ \\
\hline$D^{0}(1864)$ & $7.5 \cdot 10^{-6}$ & $K^{-} \pi^{+} \pi^{+} \pi^{-}$ & 0.075 & 0.1 & $\mathrm{y}$ & 0.37 & $2.1 \cdot 10^{-4}$ & $1.3 \cdot 10^{3}$ \\
\hline$\overline{D^{0}}(1864)$ & $2.3 \cdot 10^{-5}$ & $K^{+} \pi^{-}$ & 0.038 & 0.1 & $\mathrm{y}$ & 3.25 & $2.6 \cdot 10^{-3}$ & $1.6 \cdot 10^{4}$ \\
\hline$D^{+}(1869)$ & $8 \cdot 10^{-6}$ & $K^{-} \pi^{+} \pi^{+}$ & 0.092 & 0.1 & $\mathrm{y}$ & 4.2 & $3.1 \cdot 10^{-3}$ & $1.9 \cdot 10^{4}$ \\
\hline$D^{-}(1869)$ & $1.8 \cdot 10^{-5}$ & $K^{+} \pi^{-} \pi^{-}$ & 0.092 & 0.1 & $\mathrm{y}$ & 4.2 & $7 \cdot 10^{-3}$ & $4.2 \cdot 10^{4}$ \\
\hline$\Lambda_{c}(2285)$ & $4.9 \cdot 10^{-4}$ & $p K^{-} \pi^{+}$ & 0.05 & 0.1 & $\mathrm{y}$ & 0.5 & $1.2 \cdot 10^{-2}$ & $7.4 \cdot 10^{4}$ \\
\hline$J / \psi(3097)$ & $3.8 \cdot 10^{-6}$ & $e^{+} e^{-}$ & 0.06 & $1-10$ & $\mathrm{y}$ & 14 & $0.032-0.32$ & $1.9 \cdot 10^{5-6}$ \\
\hline$\psi^{\prime}(3686)$ & $5.1 \cdot 10^{-8}$ & $e^{+} e^{-}$ & $7.3 \cdot 10^{-3}$ & $1-10$ & $\mathrm{y}$ & 15 & $5.6 \cdot 10^{-(5-4)}$ & $3.4 \cdot 10^{2-3}$ \\
\hline$J / \psi(3097)$ & $3.8 \cdot 10^{-6}$ & $\mu^{+} \mu^{-}$ & 0.06 & 10 & $\mathrm{y}$ & 16 & 0.36 & $2.2 \cdot 10^{6}$ \\
\hline$\psi^{\prime}(3686)$ & $5.1 \cdot 10^{-8}$ & $\mu^{+} \mu^{-}$ & $7.3 \cdot 10^{-3}$ & 10 & $\bar{y}$ & 19 & $7.1 \cdot 10^{-4}$ & $4.3 \cdot 10^{5}$ \\
\hline
\end{tabular}



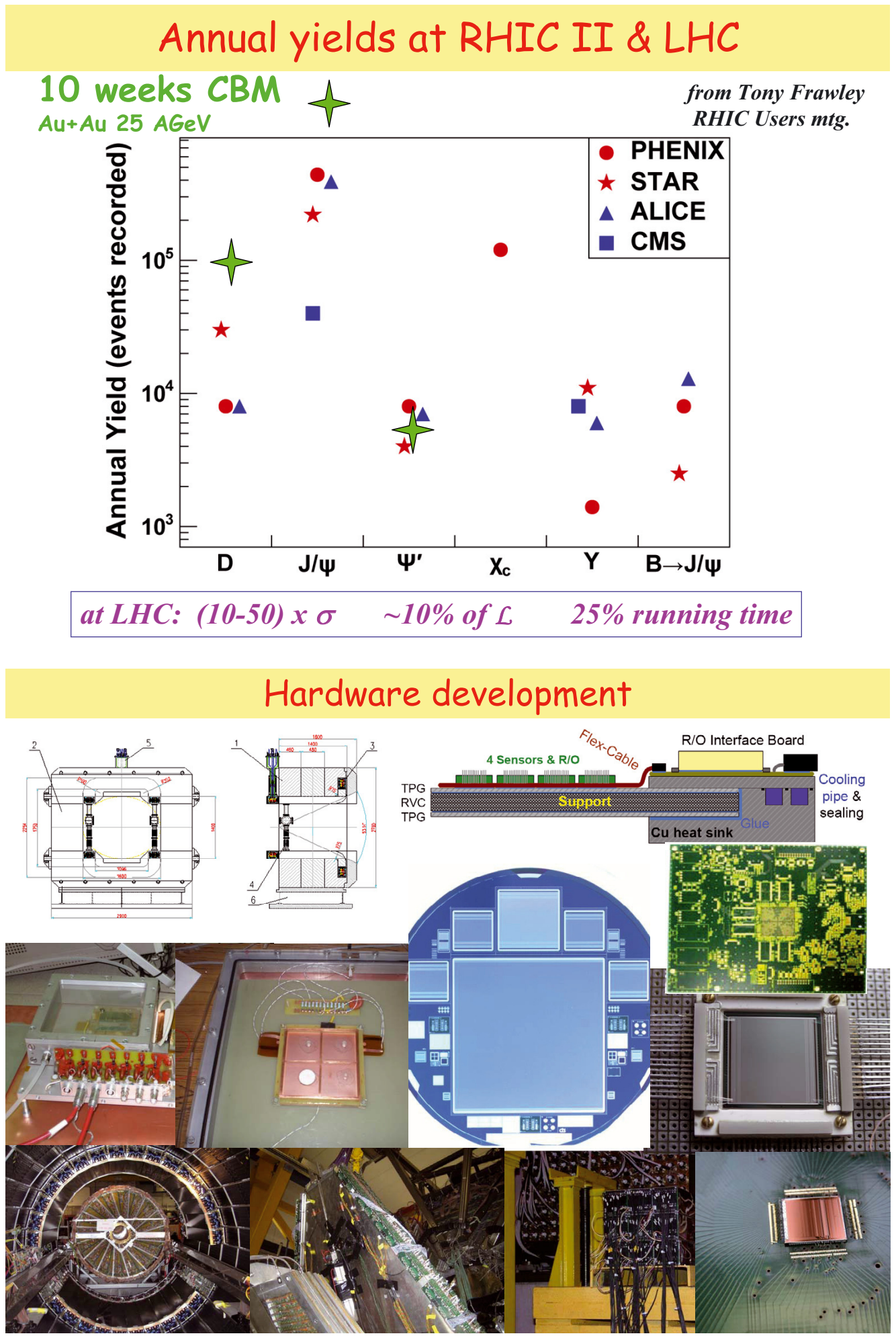


\section{CBM DAQ Architecture}
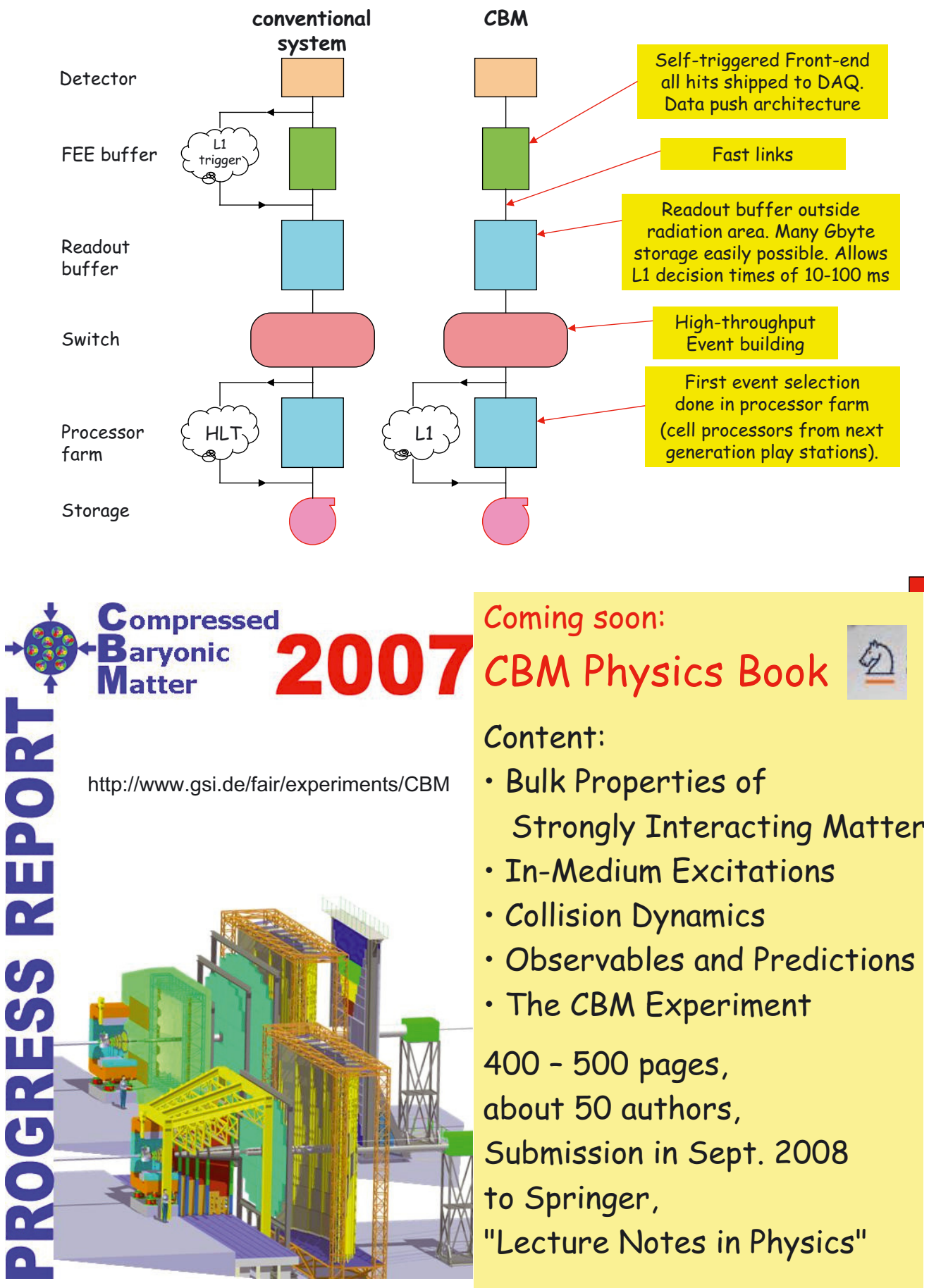


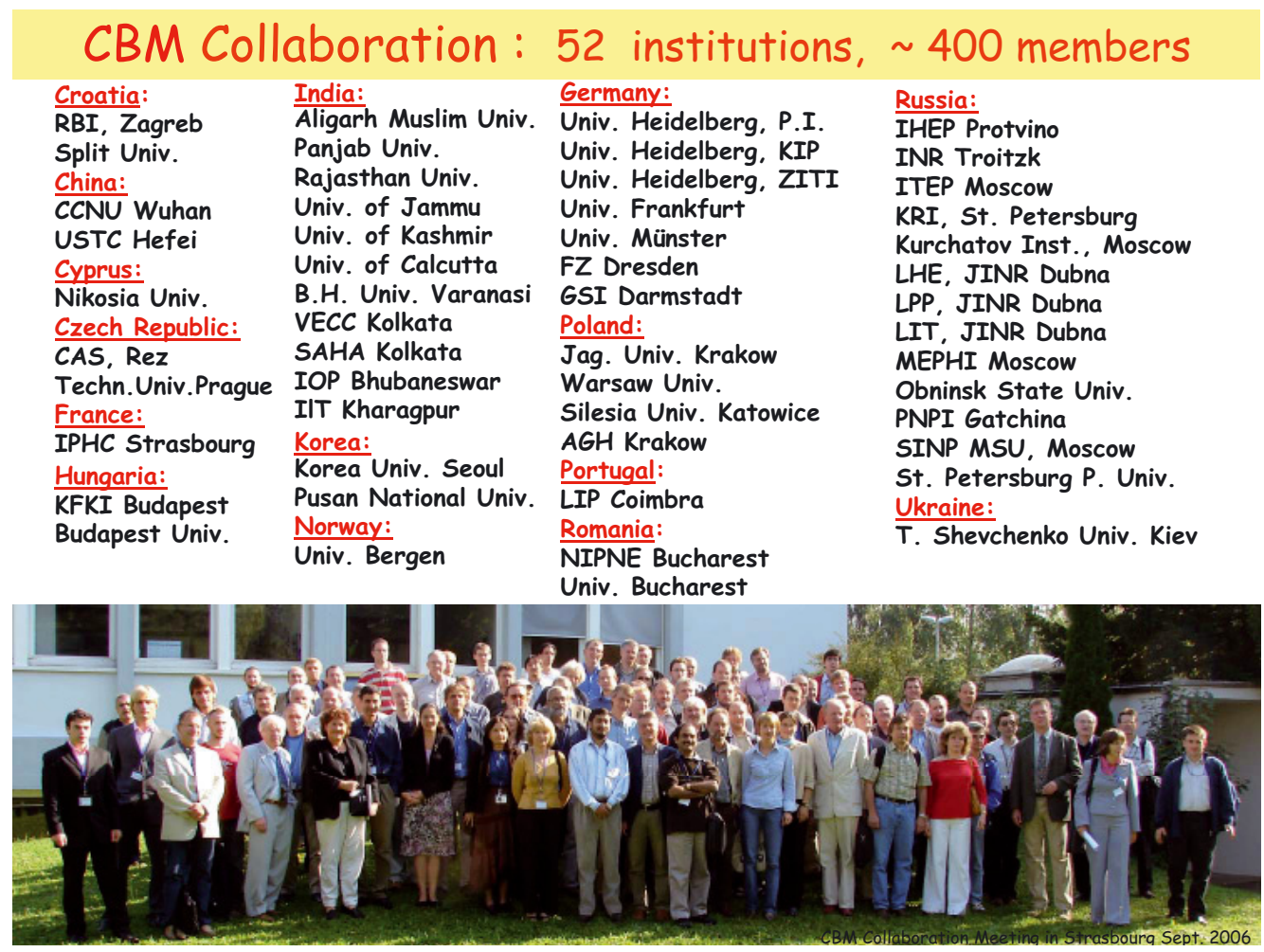




\section{Acceptance for particles identified by TOF}

Very important for the measurement of event-by-event fluctuations:

a large and azimuthally symmetric acceptance

$100 \%$ purity:
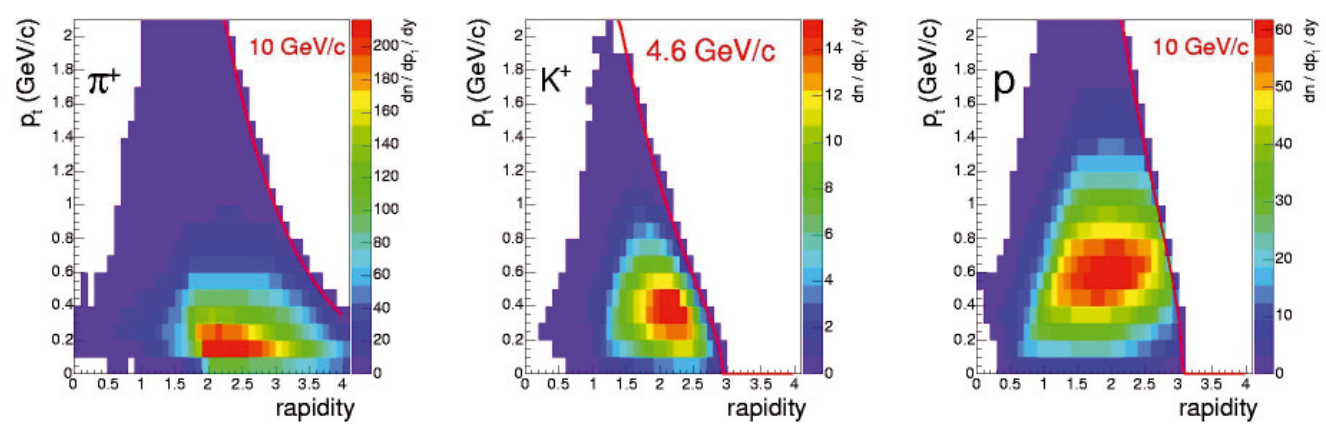

Dilepton measurements in heavy ion collisions: fixed-target versus collider experiments
1. Experimental setups
2. Multiplicities
3. Luminosities
4. Rates 


\section{Dilepton sources in Heavy-ion Collisions}

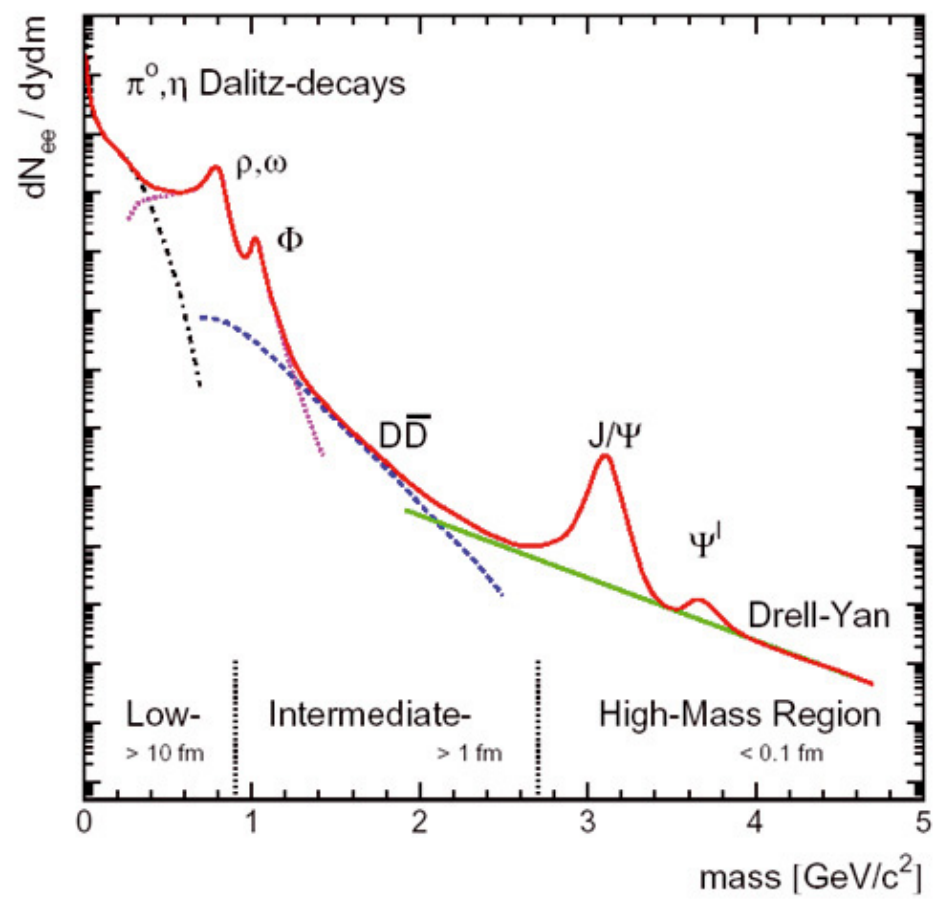

Single electron spectra central Au+Au collisions $25 \mathrm{AGeV}$

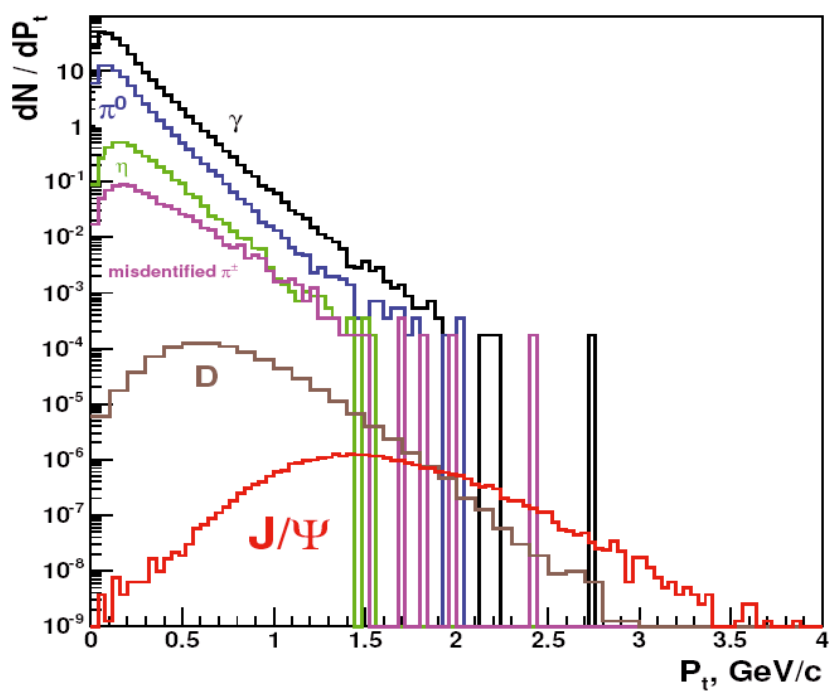

Background sources

1. external pair conversion:

$\gamma \rightarrow \mathrm{e}^{+} \mathrm{e}^{-}$

2.Dalitz-decays:

$\pi^{0} \rightarrow \gamma \mathrm{e}^{+} \mathrm{e}^{-}\left(\mathrm{BR}=1.2 \cdot 10^{-2}\right)$

$\eta \rightarrow \gamma \mathrm{e}^{+} \mathrm{e}^{-}\left(\mathrm{BR}=4.9 \cdot 10^{-3}\right)$

3. Bremsstrahlung:

pn $\rightarrow$ pn $\mathrm{e}^{+} \mathrm{e}^{-}$

4. misidentified pions

Background in muon measurements: $\Pi \rightarrow \mu \mathrm{v}, \mathrm{K} \rightarrow \mu \mathrm{v}$ $\eta \rightarrow \gamma \mu^{+} \mu^{-}$(can be determined by $\eta \rightarrow \mu^{+} \mu^{-}$) 


\section{The pioneering experiment: DLS at the Bevalac}

G. Roche et al., Phys. Lett. B 226 (1989) 228

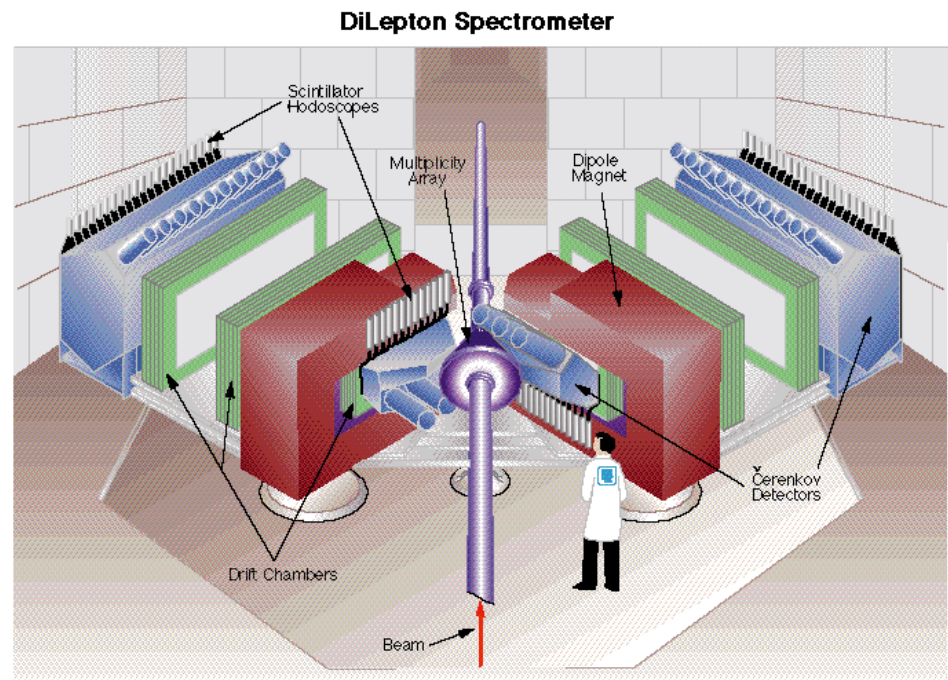

Acceptance for $e^{+} e^{-}$pairs: $0.3 \%$ Massresolution: $\Delta \mathrm{m} / \mathrm{m}=10 \%$

\section{Ring Imaging Cherenkov detector (RICH)}

Bestimmung der TeilchenGeschwindigkeit durch Messung von $\theta$ (Ringradius des Lichtkegels)

$$
\cos \theta \alpha=1 /(\beta n)
$$
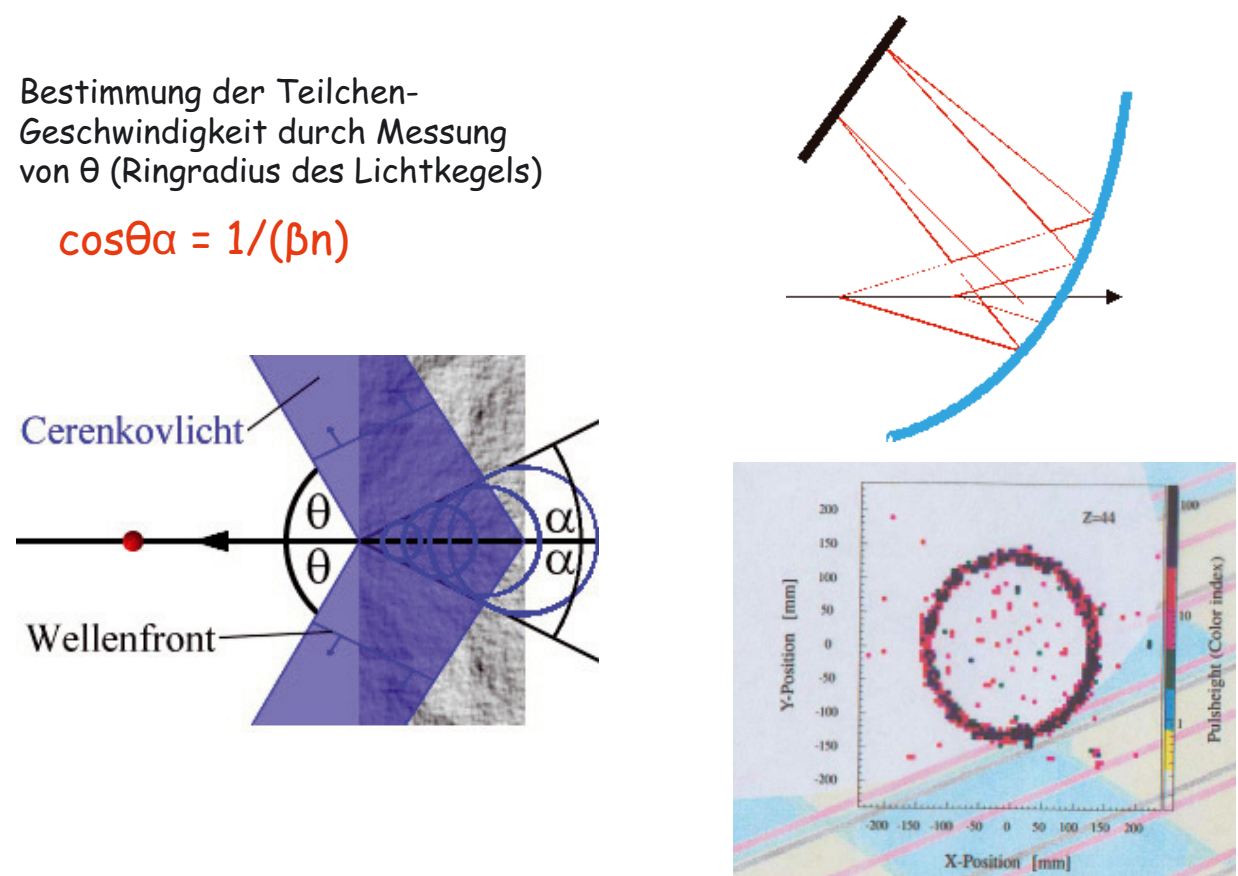


\section{DLS data}

DLS-data: R.J. Porter et al.: Phys. Rev. Lett. 79 (1997) 1229

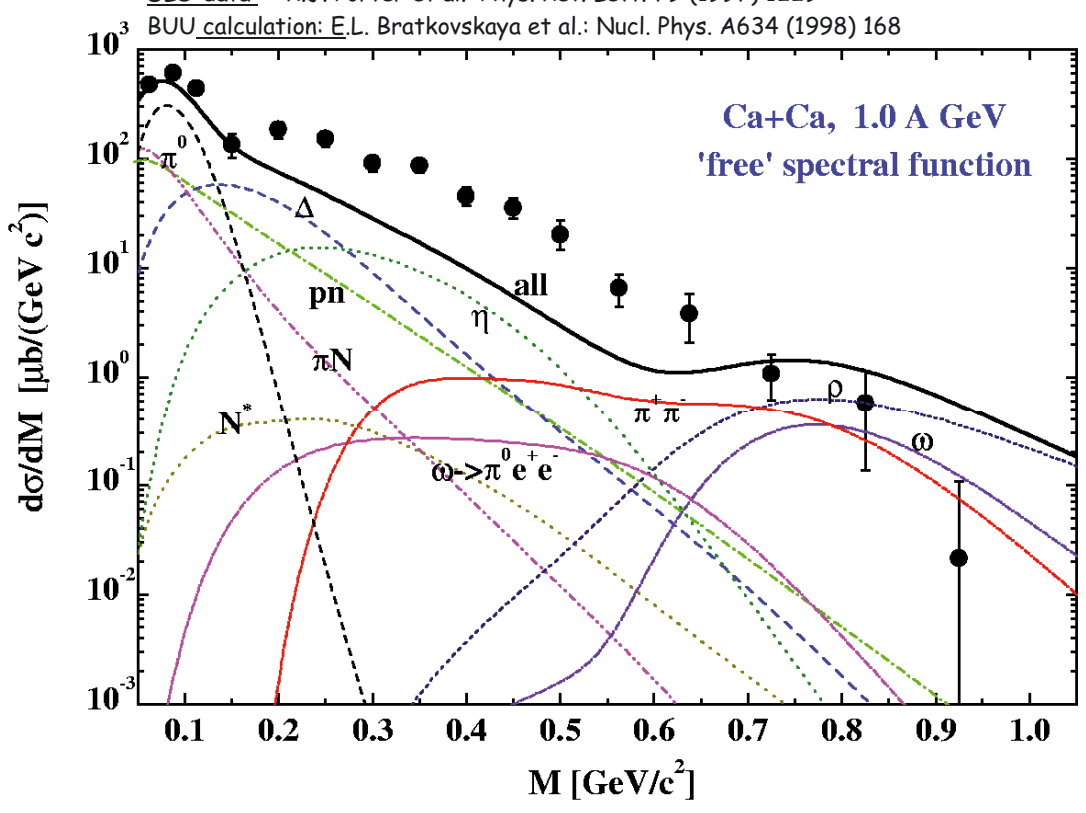

HADES at GSI
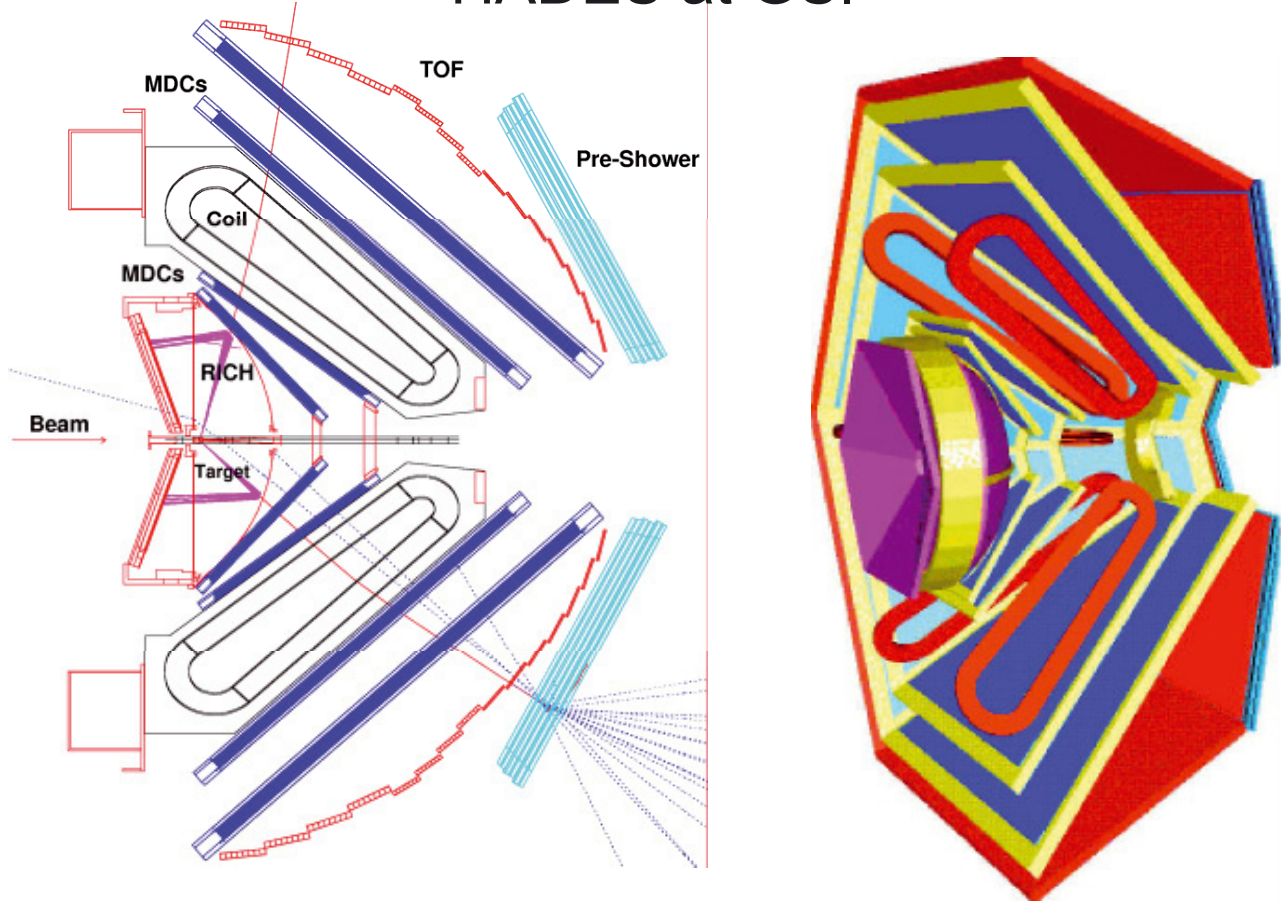
EPJ Web of Conferences

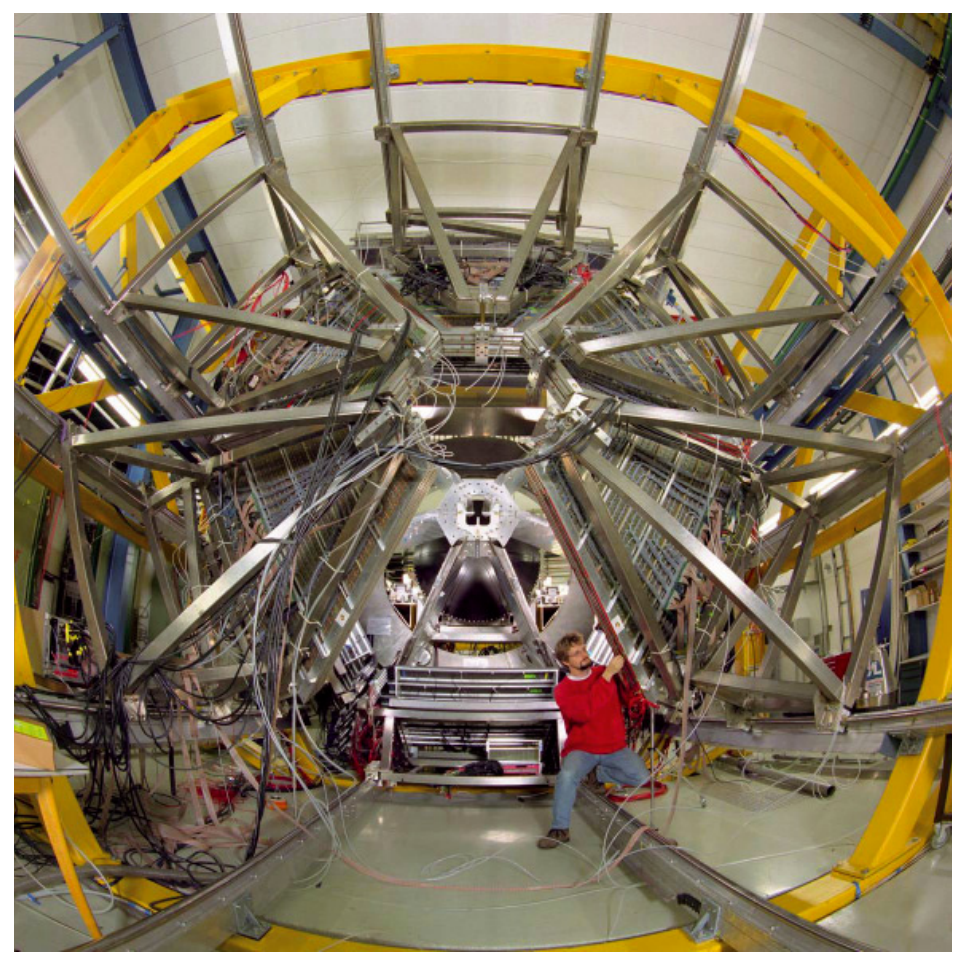

HADES

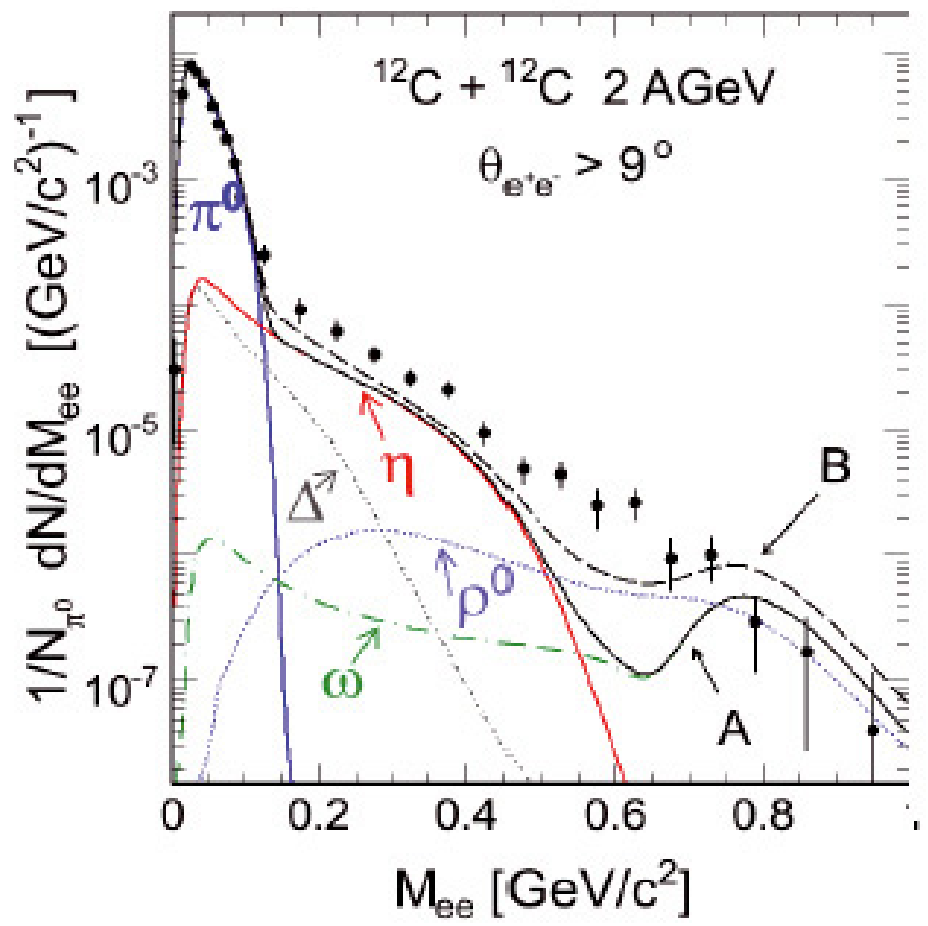

02003-p.46 


\section{CERES/NA45 at SPS}

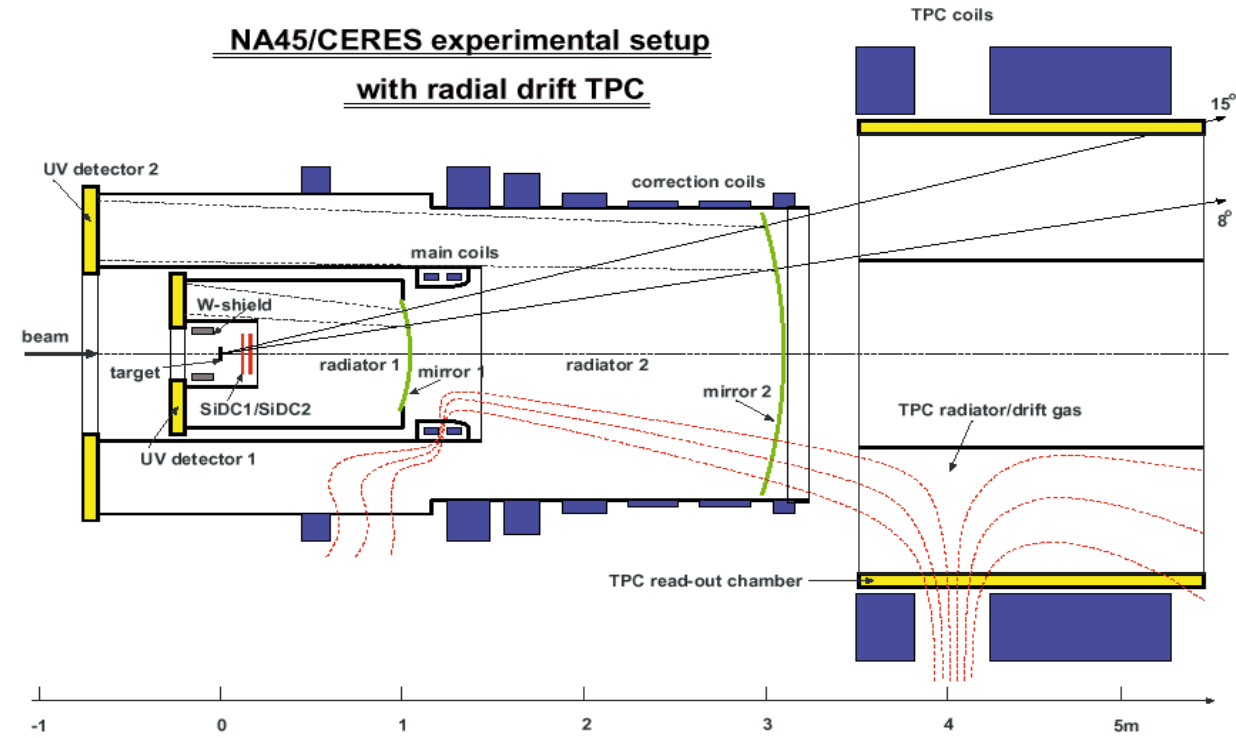

Electron-positron pairs from CERES
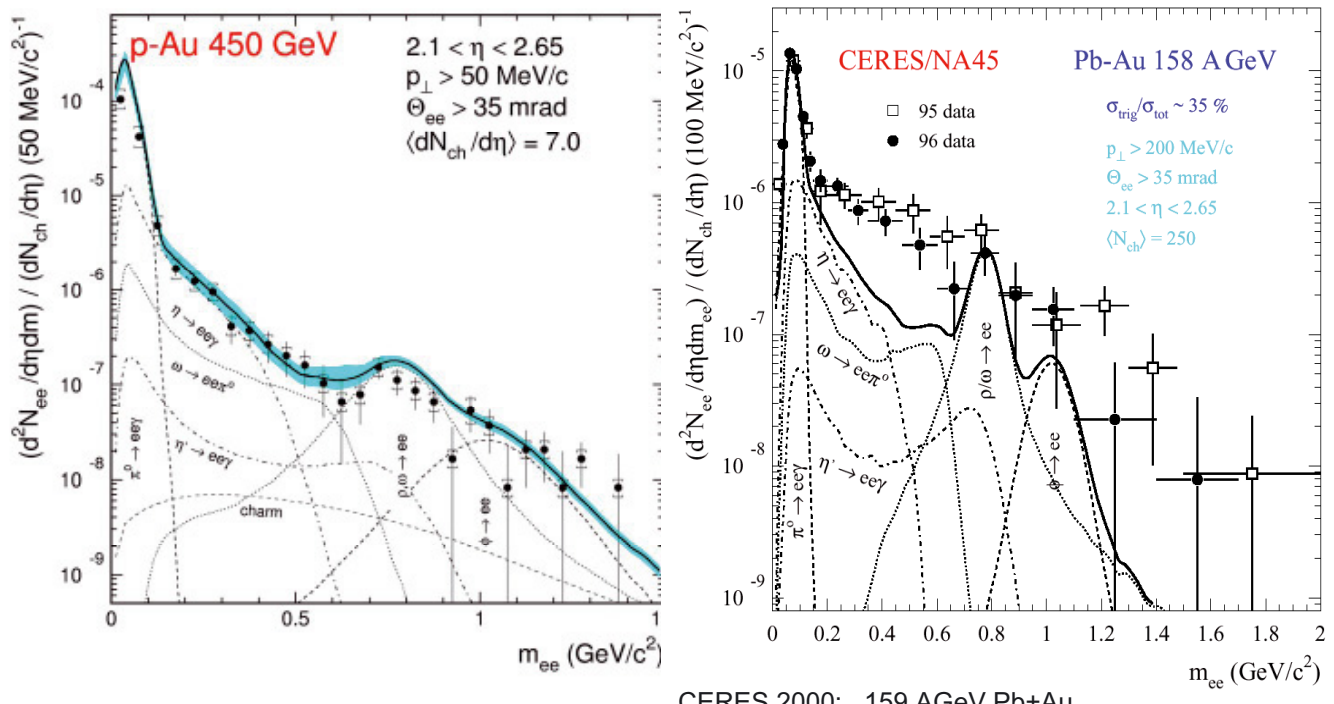

CERES 2000: $159 \mathrm{AGeV} \mathrm{Pb+Au}$ beam intensity: $10^{6}$ ions / spill 1 spill $=4 \mathrm{~s}$ beam and $15 \mathrm{~s}$ pause targets: $13 \times 25 \mu \mathrm{m} \mathrm{Au} \mathrm{(} 1 \%$ interaction) trigger: $8 \%$ most central

Event rate $=470 /$ spill $(\sim 25 \mathrm{~Hz}=15$ Mio events/week $)$ 


\section{Low mass vector mesons (CERES/CERN)}

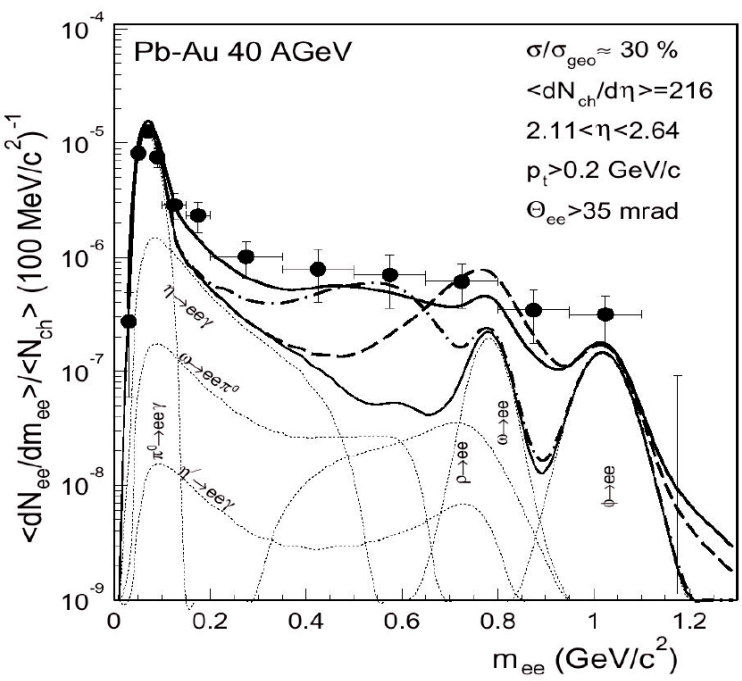

Data: 180 signal pairs

Calculations by R. Rapp:

thick dashed line:

unmodified rho

thick dashed-dotted line:

in-medium dropping rho mass

thick solid line:

in-medium spread rho width

D.Adamova et al., PRL 91 (2003) 042301

\section{Muon identification: NA38/50/60}

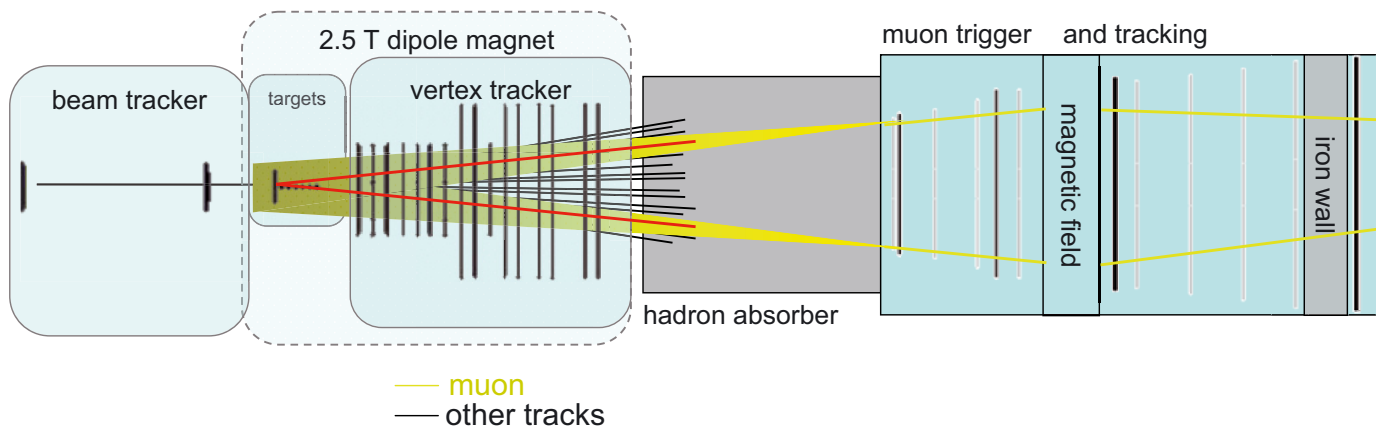

Concept of NA60: place a silicon tracking telescope in the vertex region to measure the muons before they suffer multiple scattering in the absorber and match them to the tracks measured in the muon spectrometer

$\Downarrow$

Improved kinematics; dimuon mass resolution at the $\omega$ : $\sim 20 \mathrm{MeV} / \mathrm{c}^{2}$ (instead of $80 \mathrm{MeV} / \mathrm{c}^{2}$ in NA50)

Origin of muons can be accurately determined

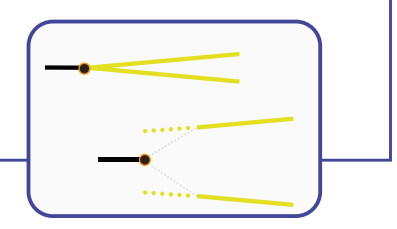




\section{Dimuon pairs measured by NA60 (CERN)}

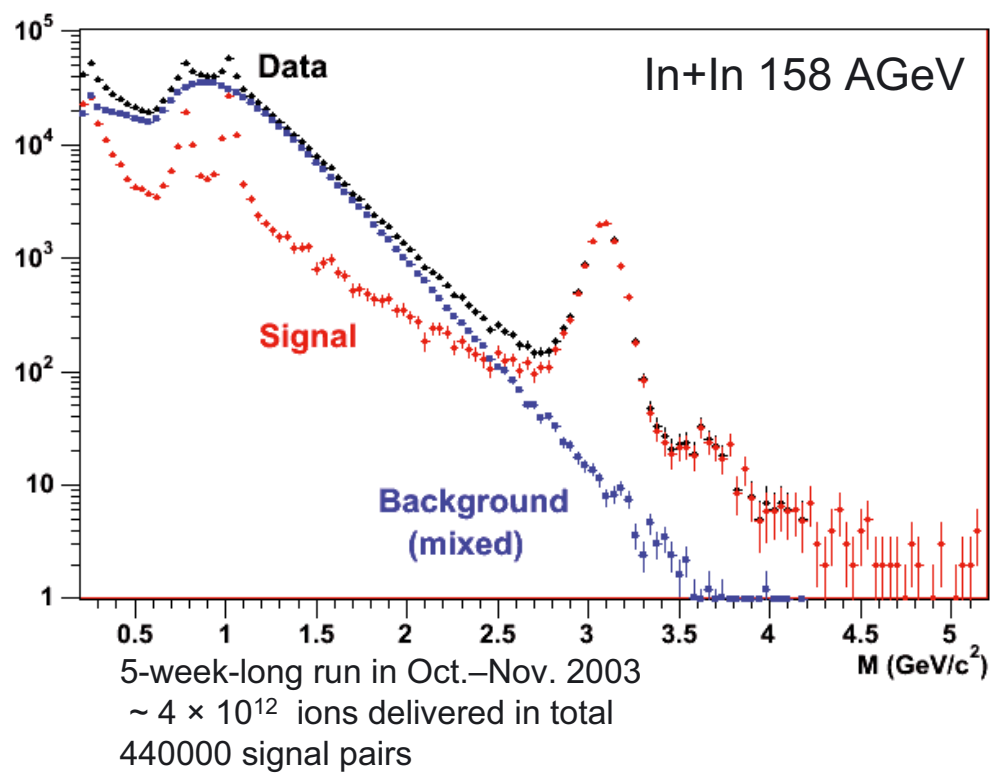

\section{$=$ Relativistic Heavy Ion Collider}

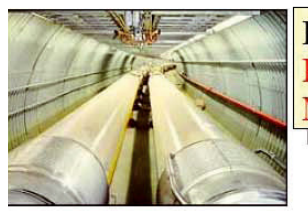

Projectile ion: e.g. $\mathrm{Au}^{79}$

Energy: 2 x $100 \mathrm{GeV} / \mathrm{u}$

Magnetic dipol field: $6 \mathrm{~T}$

$s_{N N}=\left(E_{1}+E_{2}\right)^{2}-\left(p_{1}+p_{2}\right)^{2}$

collider: $\boldsymbol{p}_{\mathbf{1}}+\mathbf{p}_{\mathbf{2}}=0 \rightarrow{\sqrt{s_{N N}}}=E_{1}+E_{2}$

fixed target: $E_{2}=m, p_{2}=0$

$\mathrm{s}_{\mathrm{NN}}=\left(\mathrm{E}_{\mathrm{kin}}+2 \mathrm{~m}\right)^{2}-\mathbf{p}_{1}^{2}$

$\mathrm{s}_{\mathrm{NN}}=2 \mathrm{~m} \cdot\left(\mathrm{E}_{\mathrm{kin}}+2 \mathrm{~m}\right)$

for $E_{k i n} \gg m: \sqrt{s_{N N}}=1.4 \cdot \sqrt{ } E_{k i n}$
If the incoming beam is simply slammed into a stationary target, much of the

energy is taken up by the target's recoil.
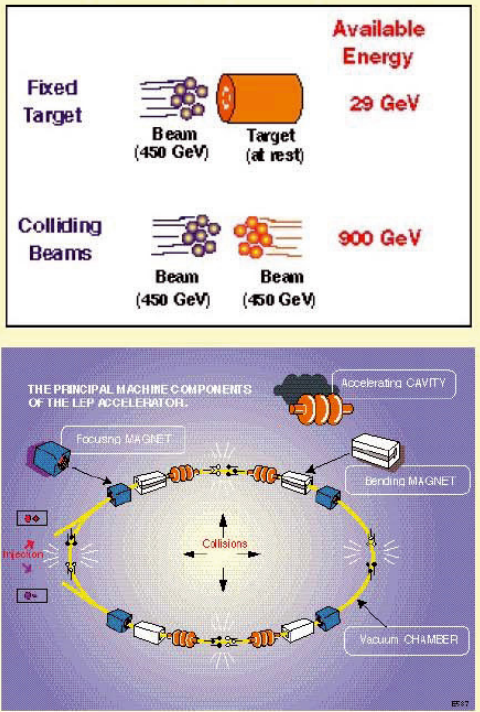


\section{PHENIX Physics Capabilities}

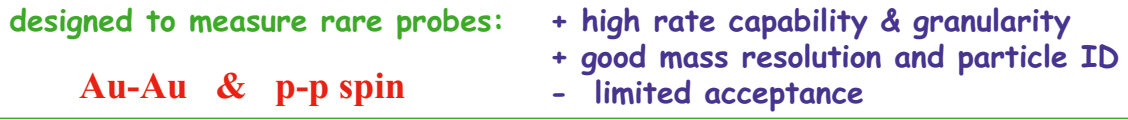

- 2 central arms:

electrons, photons, hadrons

- charmonium $\mathrm{J} / \psi, \psi^{\prime} \rightarrow \mathrm{e}^{+} e^{-}$

- vector meson $\rho, \omega, \phi \rightarrow e^{+} e^{-}$

- high $\mathrm{p}_{\mathrm{T}}$ $\pi^{\mathrm{o}}, \pi^{+}, \pi^{-}$

- direct photons

- open charm

- hadron physics

- 2 muon arms: muons

- "onium" J/ $\psi, \psi^{\prime}, \mathrm{Y} \rightarrow \mu^{+} \mu$

- vector meson $\phi \rightarrow \mu^{+} \mu^{-}$

- open charm

- combined central and muon arms:

charm production $D D->e \mu$

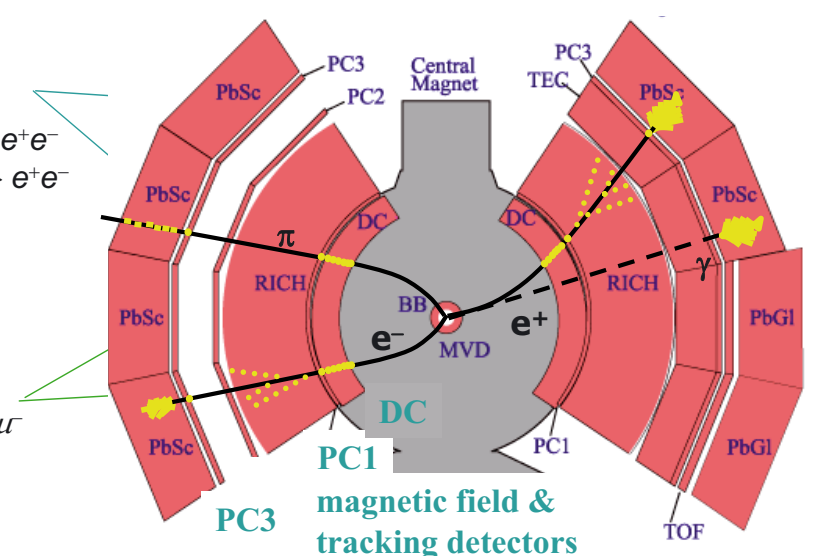

West

yiundi View

(1)

forward energy and multiplicity

- event characterization

\section{PHENIX data}

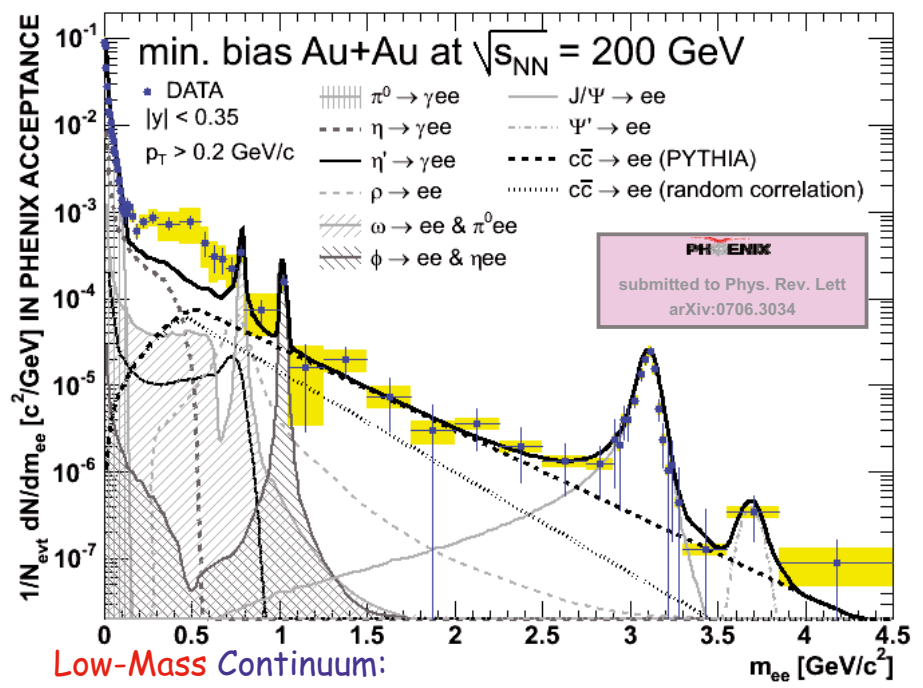

-Data absolutely normalized

-Cocktail filtered in PHENIX acceptance

- Charm from

- PYTHIA

- Single electron non photonic spectrum w/o angular correlations

$\sigma_{\mathrm{c}}=\mathrm{N}_{\text {coll }} \times 567 \pm 57 \pm 193 \mu \mathrm{b}$

enhancement $150<m_{e e}<750$ MeV: $3.4 \pm 0.2$ (stat.) \pm 1.3 (syst.) \pm 0.7 (model)

Intermediate-Mass Continuum:

Single-e $\rightarrow$ pt suppression \& non-zero v2: charm thermalized?

PYTHIA single-e $p_{T}$ spectra softer than $p+p$ but coincide with $\mathrm{Au}+\mathrm{Au}$

Angular correlations unknown

Room for thermal contribution? 


\section{CERN and the Large Hadron Collider (LHC)}

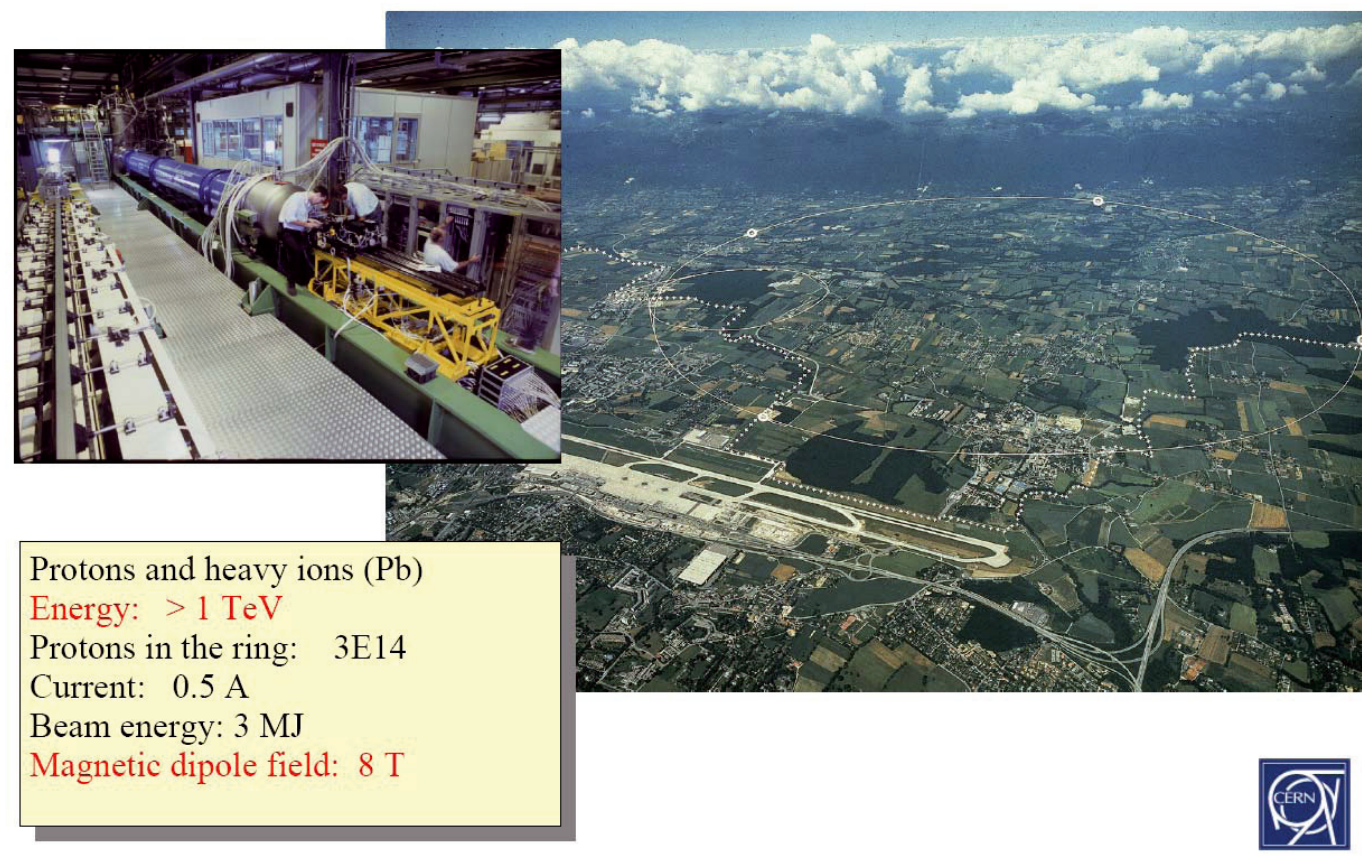

\section{The ALICE experiment at CERN}

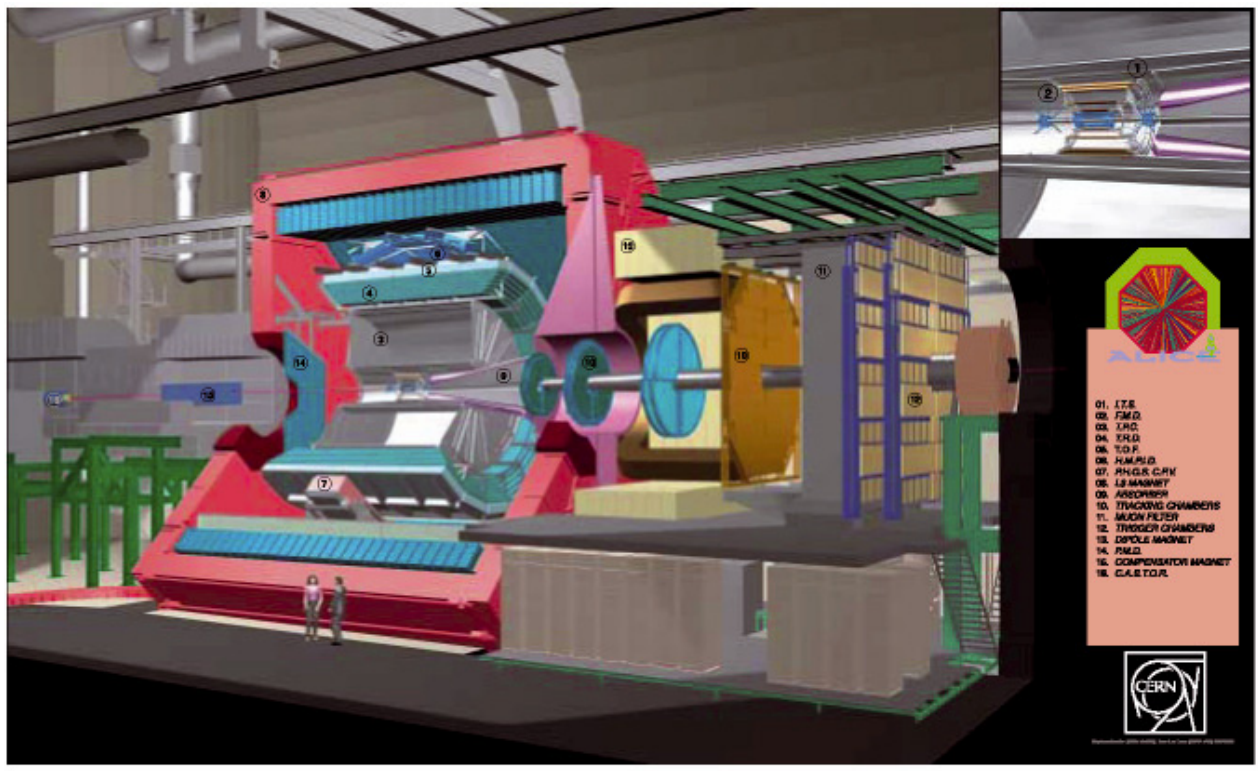

Fig.1: Layout of the ALICE detector. The Transition Radiation Detector (4) is the cyan component placed between the TPC (3) and TOF (5). 


\section{Transition radiation}

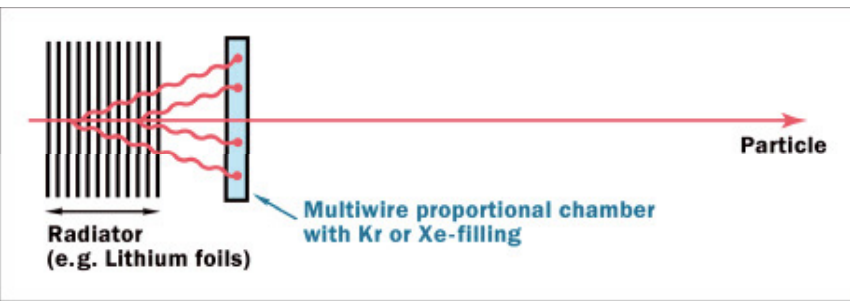

Total energy $\propto Y$ $\Theta=1 / \mathrm{V}$
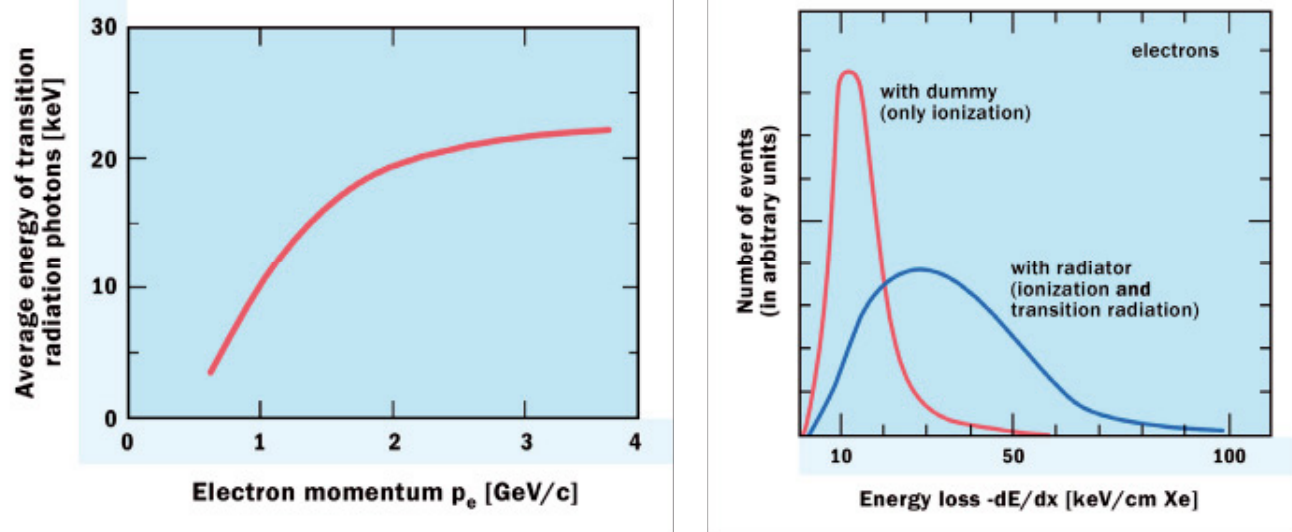

\section{Transition Radiation Detectors (TRD)}
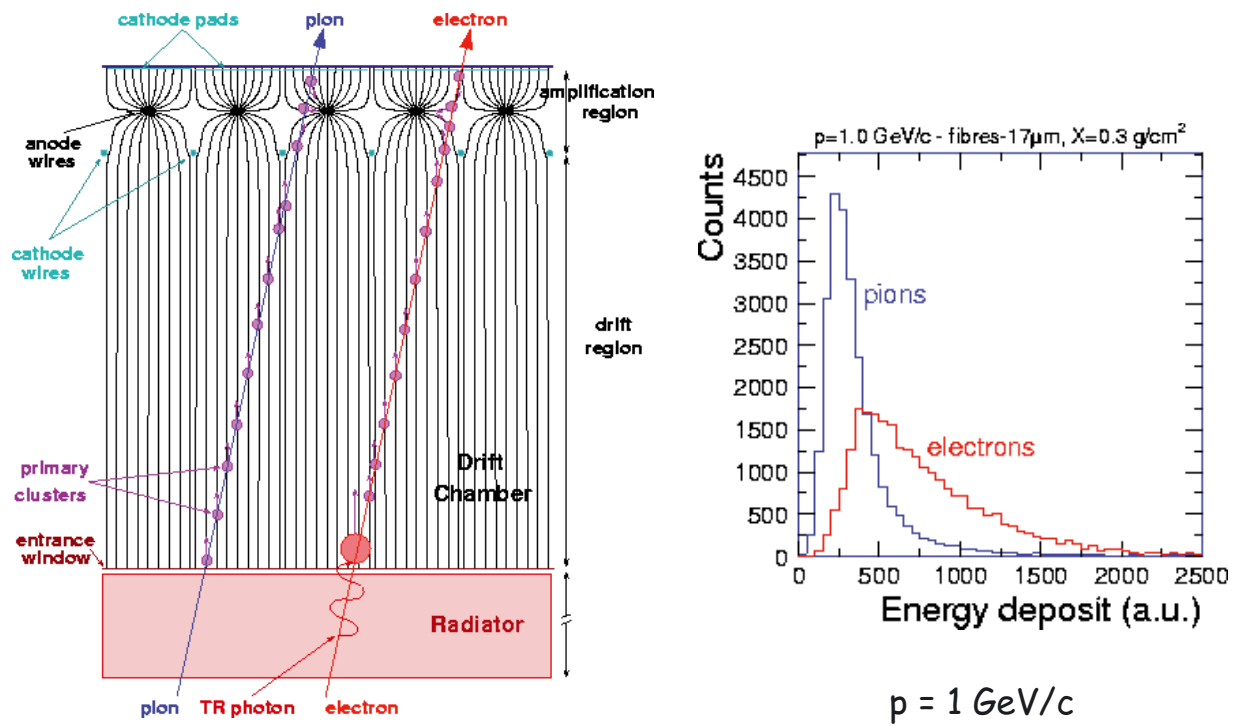

$$
\begin{aligned}
& p=1 \mathrm{GeV} / \mathrm{c} \\
& V_{e}=2000 \\
& V_{\pi}=7.1
\end{aligned}
$$




\section{Facility for Antiproton and Ion Research (FAIR)}

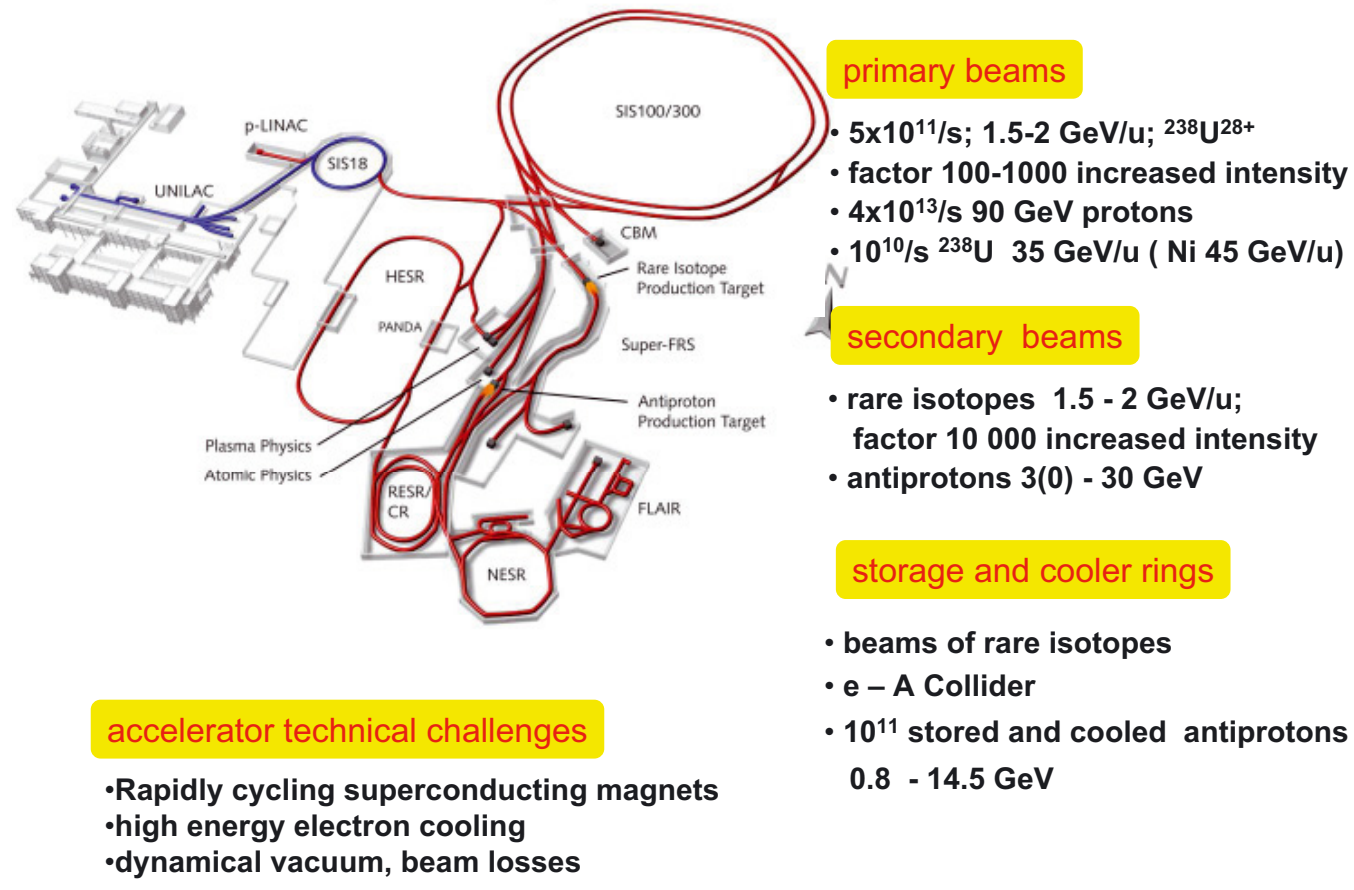

\section{The Compressed Baryonic Matter Experiment}

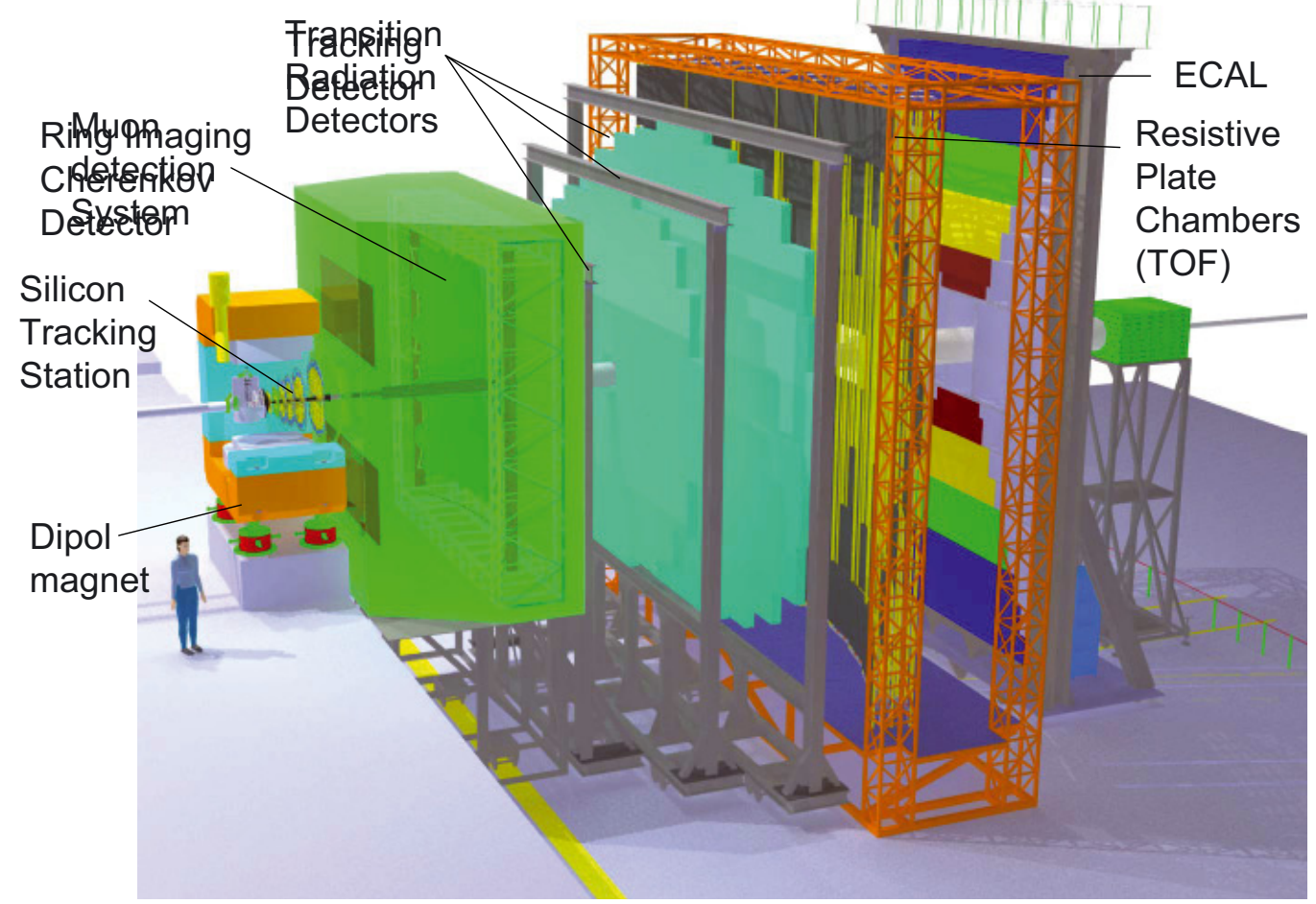




\section{Electron identification with RICH and TRD}

R vs p (after distance cut)
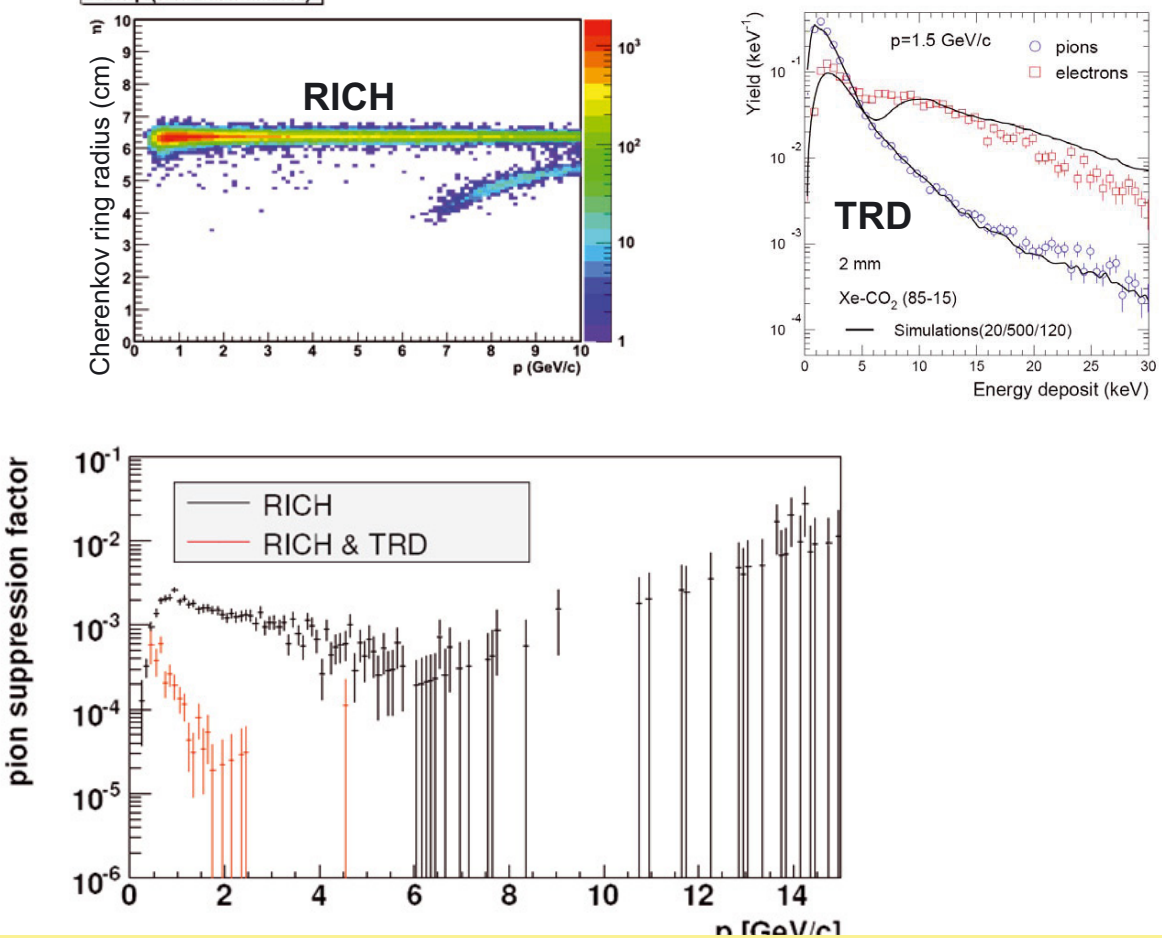

\section{Mapping the QCD phase diagram with heavy-ion collisions}

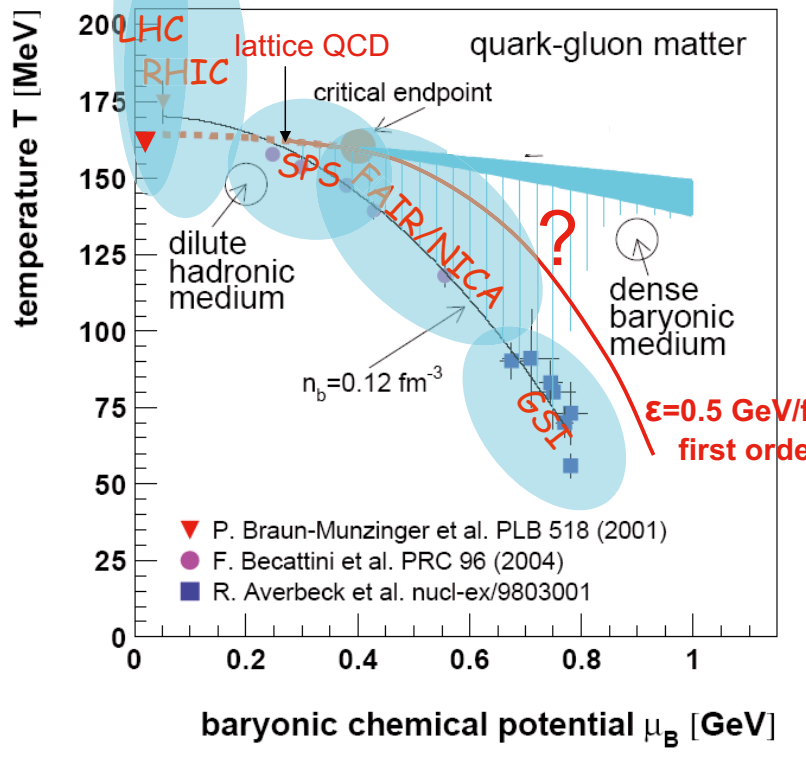

Critical endpoint:

Z. Fodor, S. Katz, hep-lat/0402006

S. Ejiri et al., hep-lat/0312006 crossover at small $\mu_{B}$

Recent $L Q C D$ calculations:

$\mathrm{T}_{\mathrm{C}}=150-190 \mathrm{MeV}$

baryon density:

$\rho_{B} \approx 4(m T / 2 \pi)^{3 / 2} \times$

$\left[\exp \left(\left(\mu_{B}-m\right) / T\right)-\exp \left(\left(-\mu_{B}-m\right) / T\right)\right]$ baryons - antibaryons 


\section{Meson production in central Au+Au collisions}

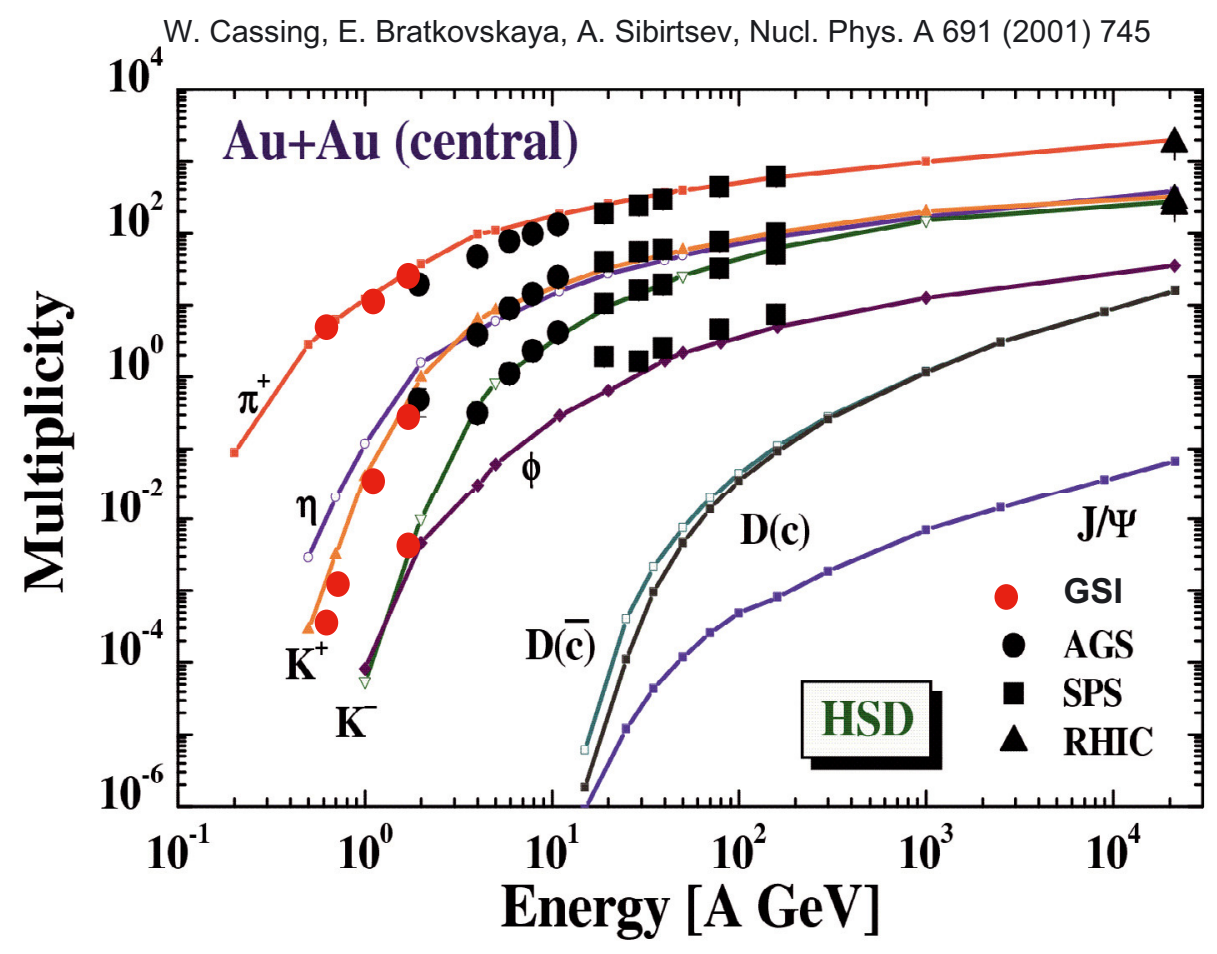

Vector meson yields for central Au+Au collisions at $\sqrt{s_{\mathrm{NN}}}=7.1 \mathrm{GeV}(25 \mathrm{AGeV})$

\begin{tabular}{|c|c|c|c|c|}
\hline & $\mathrm{J} / \Psi$ & $\rho$ & $\omega$ & $\varphi$ \\
\hline multiplicity & $2 \cdot 10^{-5}$ & 23 & 38 & 1.3 \\
\hline $\mathrm{BR}(\rightarrow \mu \mu)$ & 0.06 & $4.6 \cdot 10^{-5}$ & $9 \cdot 10^{-5}$ & $3 \cdot 10^{-5}$ \\
\hline $\begin{array}{c}\mu \mu \\
\text { multiplicity }\end{array}$ & $1.2 \cdot 10^{-6}$ & $1 \cdot 10^{-3}$ & $3.4 \cdot 10^{-3}$ & $3.7 \cdot 10^{-4}$ \\
\hline $\begin{array}{c}\mu \mu \\
\text { min bias }\end{array}$ & $3 \cdot 10^{-7}$ & $2.5 \cdot 10^{-4}$ & $8 \cdot 10^{-4}$ & $9 \cdot 10^{-5}$ \\
\hline
\end{tabular}




\section{Collider Luminosity: $L=N_{1}{ }^{*} N_{2} \cdot B / F\left[\mathrm{~cm}^{-2} \mathrm{~s}^{-1}\right]$}

$N_{1}, N_{2}$ = beam particles per bunch

$\mathrm{B}=$ number of bunch crossings per sec

$\mathrm{F}=$ beam size in $\mathrm{cm}^{2}$

Typical numbers:

$\mathrm{N}_{1}=\mathrm{N}_{2}=10^{9}$

$\mathrm{B}=10^{6}$

$\mathrm{F}=10^{-3} \mathrm{~cm}^{2}$

Reaktion rate $\mathrm{R}=\mathrm{L} \cdot \sigma$

$\sigma=$ reaction cross section

$\sigma=\pi \cdot(2 \cdot R)^{2}=4 \pi \cdot\left(r_{0} \cdot A^{1 / 3}\right)^{2}$ with $r_{0}=1.2 \mathrm{fm}$

Au+Au collisions: $A=197 \rightarrow \sigma=6$ barn, 1 barn $=10^{-24} \mathrm{~cm}^{2}$

Collider reaction rates for $\mathrm{Au}+\mathrm{Au}$ :

$\mathrm{R}=10^{27} \mathrm{~cm}^{-2} \mathrm{~s}^{-1} \cdot 6 \cdot 10^{-24} \mathrm{~cm}^{2}=6000 \mathrm{~s}^{-1}$

Fixed target Luminosity: $L=N_{B} \cdot N_{T} / F\left[\mathrm{~cm}^{-2} \mathrm{~S}^{-1}\right]$

$\mathrm{N}_{\mathrm{B}}$ = beam particles $/ \mathrm{sec}$

$\mathrm{N}_{\mathrm{T}} / \mathrm{F}=$ target atoms $/ \mathrm{cm}^{2}=\mathrm{N}_{\mathrm{A}} \cdot \rho \cdot \mathrm{d} / \mathrm{A}$

with Avogadros Number $N_{A}=6.02 \cdot 10^{23} \cdot \mathrm{mol}^{-1}$,

material density $\rho\left[\mathrm{g} / \mathrm{cm}^{3}\right]$,

target thickness $\mathrm{d}[\mathrm{cm}]$

atomic number $\mathrm{A}$

Typical numbers:

$\mathrm{N}_{\mathrm{B}}=10^{9} \mathrm{~s}^{-1}$

Au target: $\rho=19.3 \mathrm{~g} / \mathrm{cm}^{3}, A=197$

$\mathrm{d}=0.3 \mathrm{~mm}$ ( $1 \%$ interaction rate)

$\mathrm{L}=1.8 \cdot 10^{30} \mathrm{~cm}^{-2} \mathrm{~s}^{-1}$

Fixed target reaction rates for $\mathrm{Au}+\mathrm{Au}$ :

$\mathrm{R}=\mathrm{L} \cdot \sigma=1.8 \cdot 10^{30} \mathrm{~cm}^{-2} \mathrm{~s}^{-1} \cdot 6 \cdot 10^{-24} \mathrm{~cm}^{2}=10^{7} \mathrm{~s}^{-1}$ 


\section{Acceptances and Efficiencies}

$\varepsilon=\varepsilon_{\Delta \Omega} \cdot \varepsilon_{\Delta \mathrm{p}}{ }^{\prime} \varepsilon_{\text {Det }} \cdot \varepsilon_{\text {Trigg }}{ }^{\prime} \varepsilon_{\text {DAQ }}{ }^{\prime} \varepsilon_{\text {analysis }}$

with

$\varepsilon_{\Delta \Omega}=$ angular acceptance

$\varepsilon_{\Delta \mathrm{p}}=$ momentum acceptance

$\varepsilon_{\text {Det }}=$ detector efficiencies

$\varepsilon_{\text {Trigg }}=$ trigger efficiencies

$\varepsilon_{\mathrm{DAQ}}=$ dead time correction of DAQ

$\varepsilon_{\text {analysis }}=$ efficiency of analysis

(track finding, cuts for background suppression , ...)

Typical values:

$\varepsilon_{\Delta \Omega} \approx 0.5, \varepsilon_{\Delta \mathrm{p}} \approx 0.8, \varepsilon_{\text {Det }} \approx 0.9, \varepsilon_{\text {Trigg }} \approx 0.9, \varepsilon_{\mathrm{DAQ}} \approx 0.5, \varepsilon_{\text {analysis }} \approx 0.3$,

$\varepsilon \approx 0.05$

\section{Low-energy RHIC run at $\sqrt{S}_{\mathrm{NN}}=9 \mathrm{GeV}$}

peak luminosity $\sim 2 \cdot 10^{23} \mathrm{~cm}^{-2} \mathrm{~s}^{-1}$

Reaction rate $\mathrm{Au}+\mathrm{Au} \sim 1 \mathrm{~Hz}$

further reduction:

average luminosity, large diamond

improvement by upgrades incl. e- cooling

NICA collider

luminosity design value $\sim 1 \cdot 10^{27} \mathrm{~cm}^{-2} \mathrm{~s}^{-1}$ 
Expected dilepton yields for

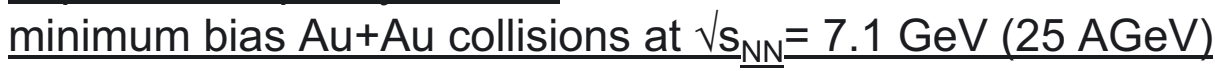

Assumption: experimental efficiency $\varepsilon=10 \%$

Multiplicity of $\mathrm{J} / \Psi$ : $M \cdot \varepsilon=3 \cdot 10^{-8}$

Multiplicity of $\omega: \quad M \cdot \varepsilon=8 \cdot 10^{-5}$

Collider reaction rate $100 \mathrm{~s}^{-1}$

Yield of $\mathrm{J} / \Psi$ :

$3 \cdot 10^{-8} \cdot 100 \mathrm{~s}^{-1}=3 \cdot 10^{-6} \mathrm{~s}^{-1}=1.1 \cdot 10^{-2} \mathrm{~h}^{-1}=19$ in 10 weeks

Yield of $\omega$ :

$8 \cdot 10^{-5} \cdot 100 \mathrm{~s}^{-1}=8 \cdot 10^{-3} \mathrm{~s}^{-1}=29 \mathrm{~h}^{-1}=50000$ in 10 weeks

Fixed target reaction rates:

$10^{7} \mathrm{~s}^{-1}$ with $\mathrm{J} / \psi$ trigger: $1.9 \cdot 10^{6} \mathrm{~J} / \Psi$ in 10 weeks

$10^{5} \mathrm{~s}^{-1}$ without trigger: Yield of $\omega: 5 \cdot 10^{7}$ in 10 weeks

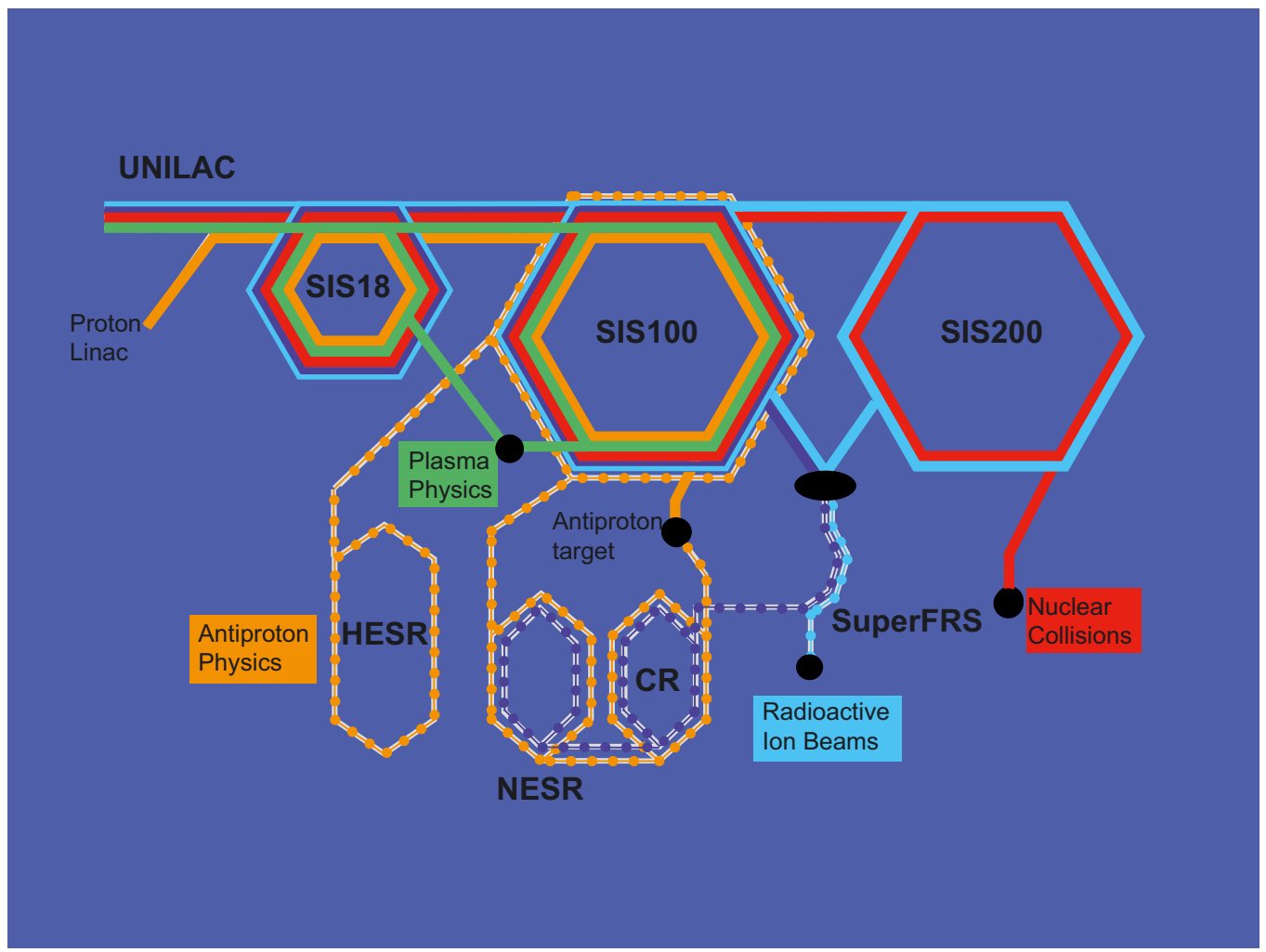




\section{Ions for RIB experiments}

\section{UNILAC}

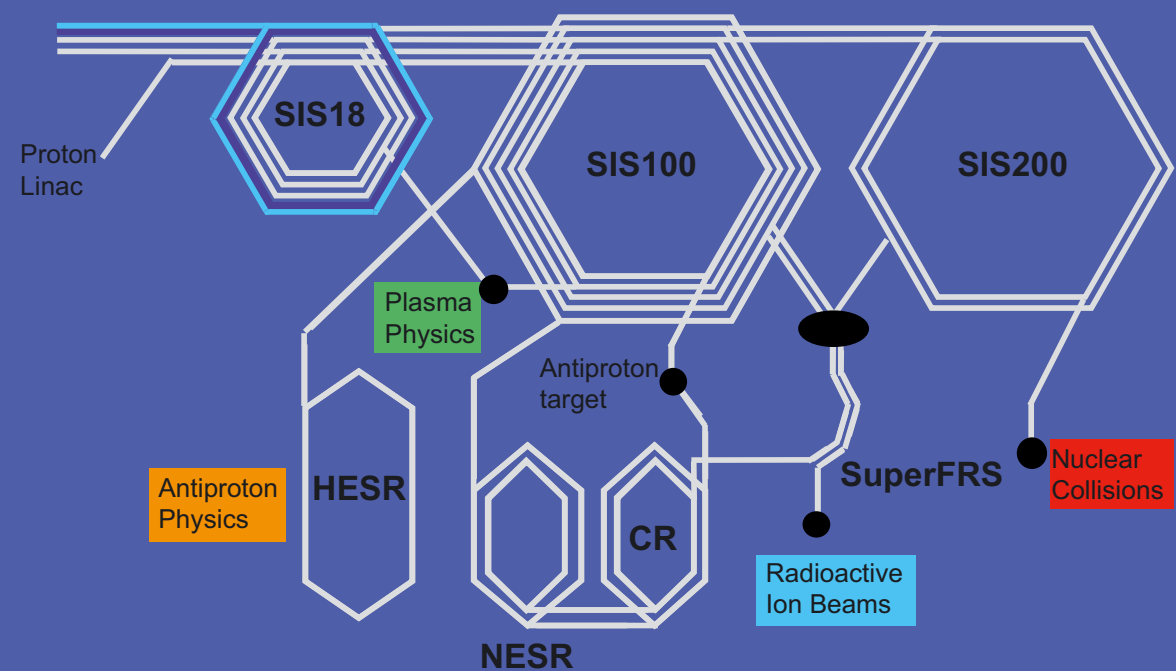

\section{lons for RIB experiments}

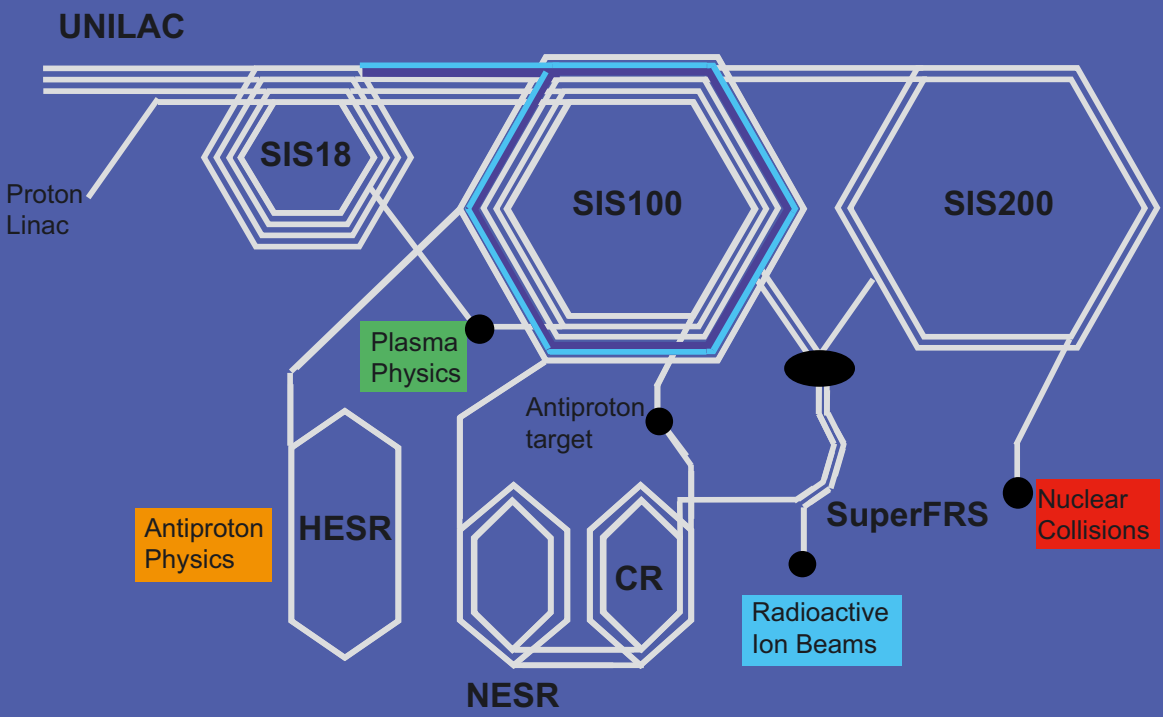




\section{Ions for RIB experiments: storage rings}

\section{UNILAC}

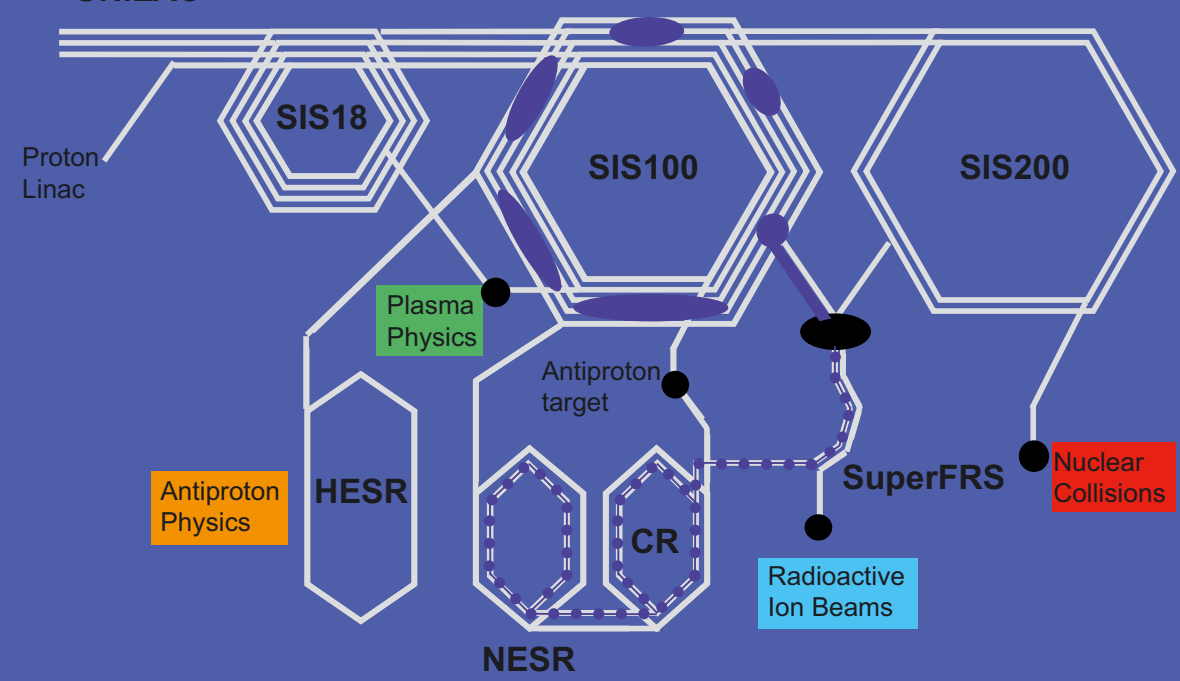

U)

\section{lons for RIB experiments: fixed target}

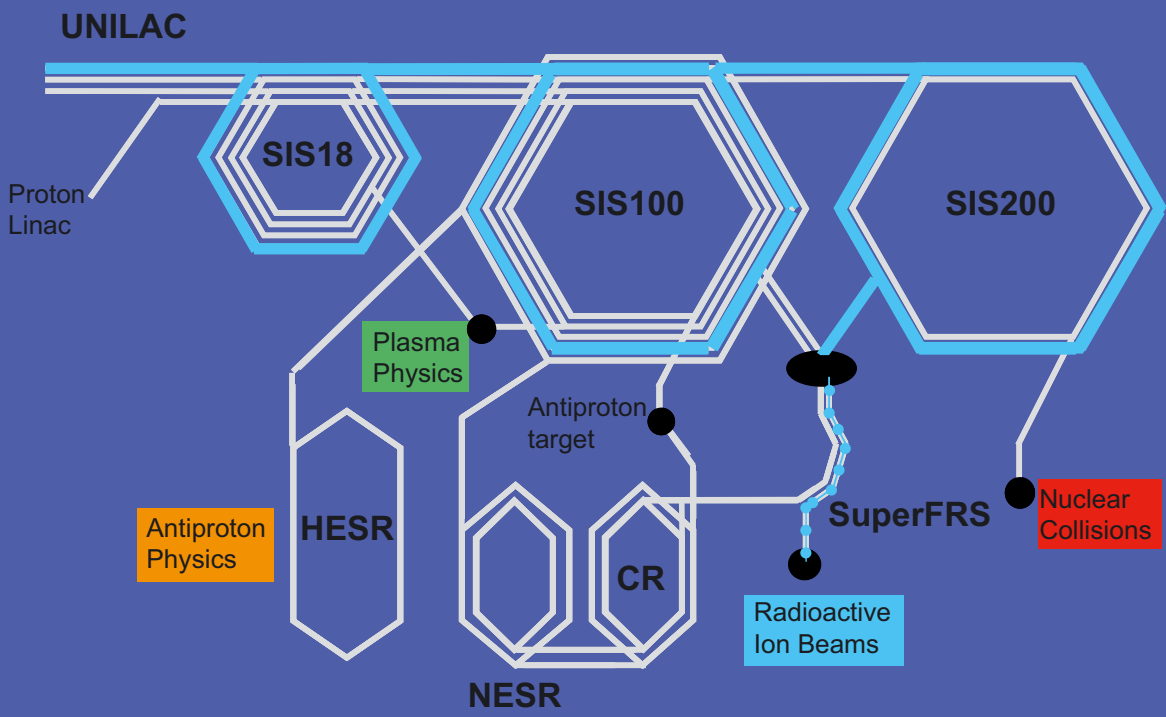




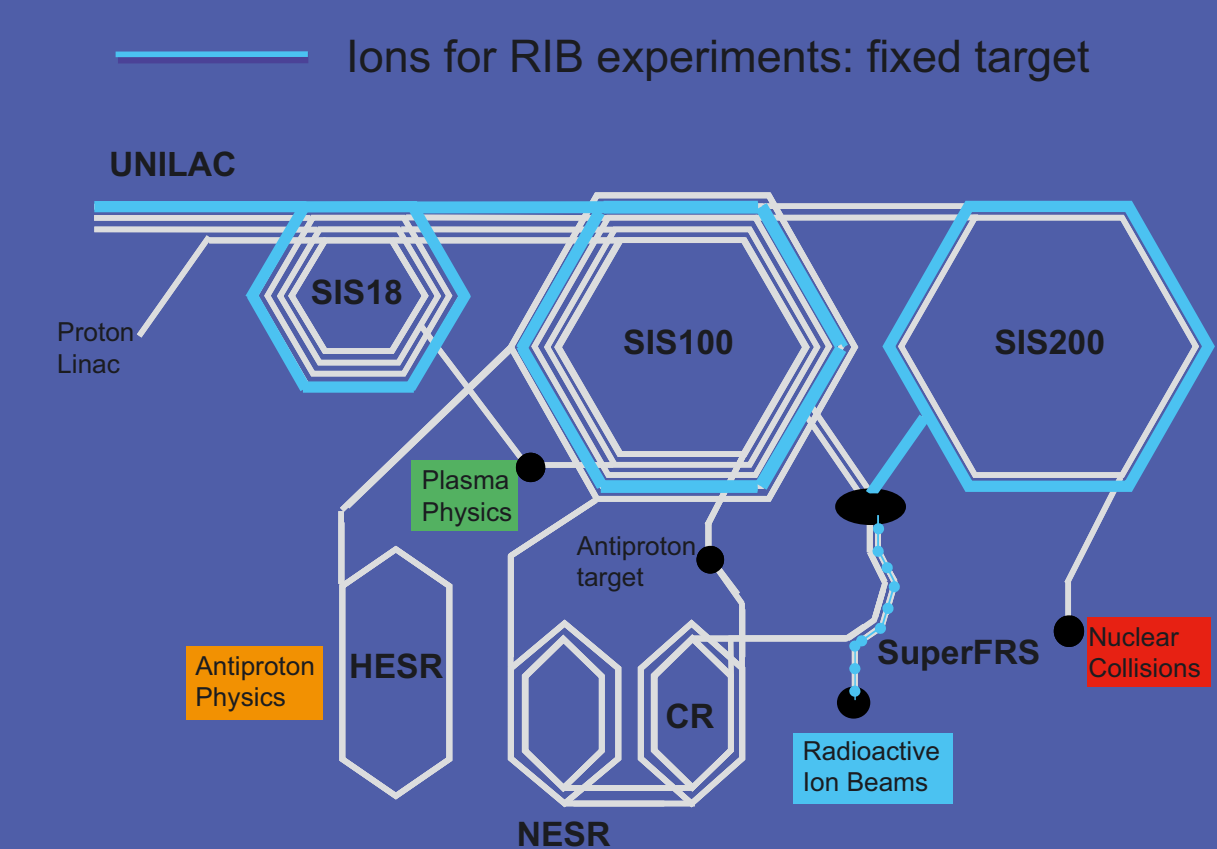

\section{Ions for RIB experiments: fixed target}

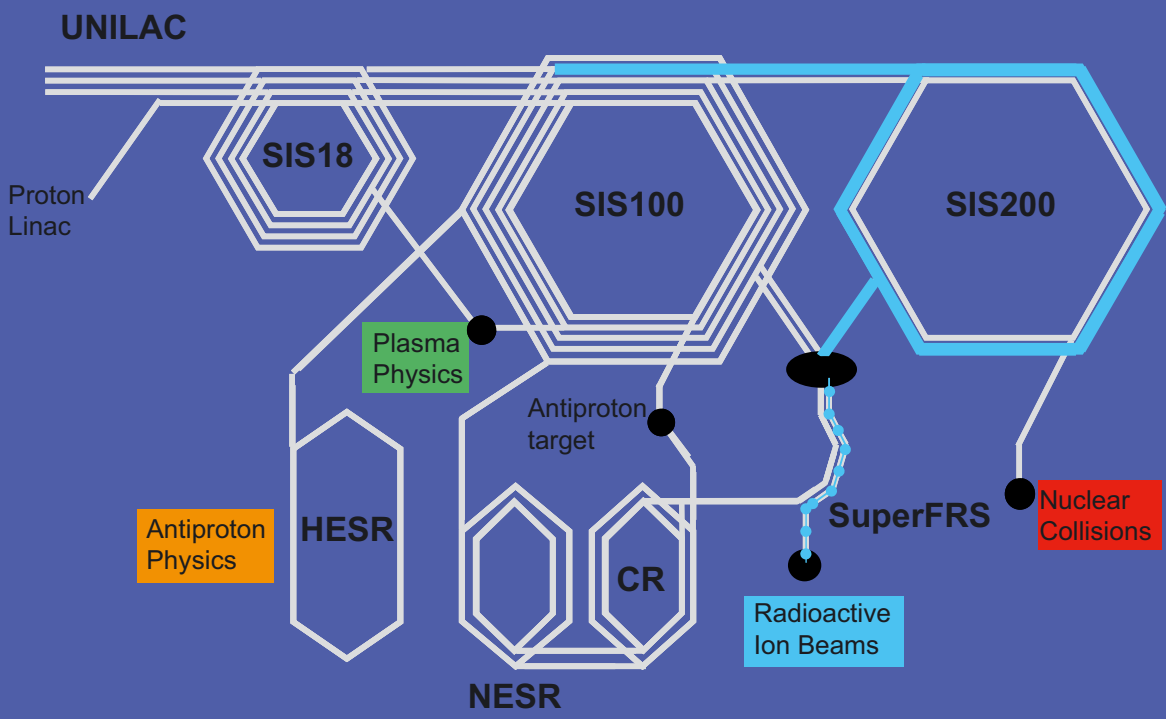




\section{Ions for RIB experiments: fixed target}

\section{UNILAC}

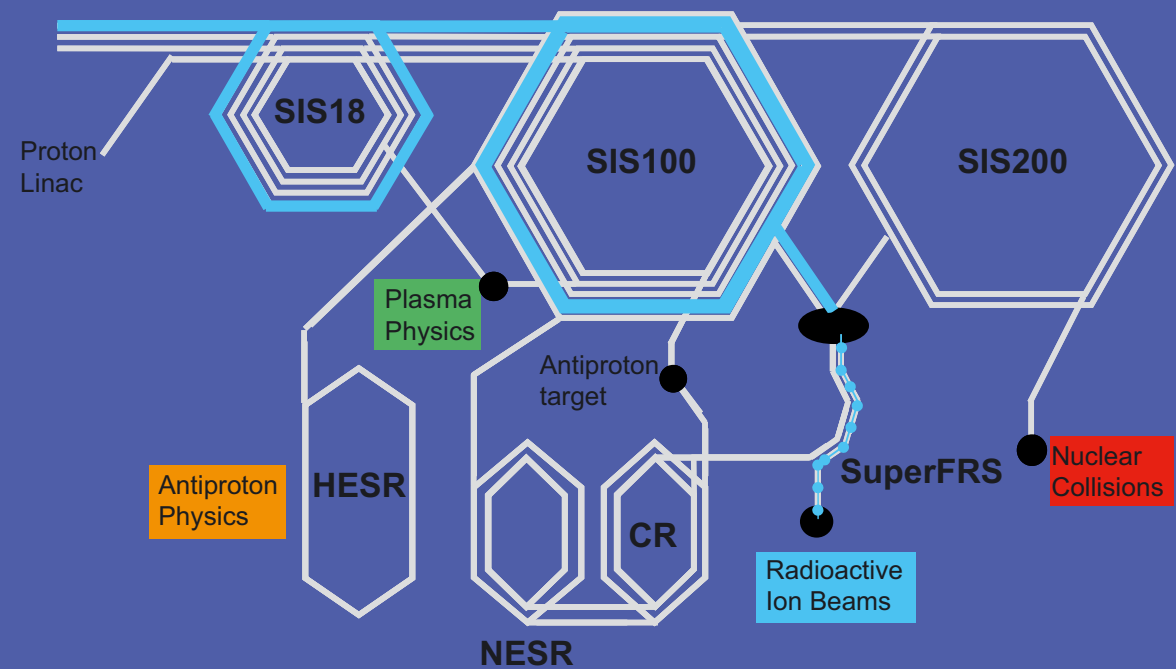

Ions for RIB experiments: fixed target

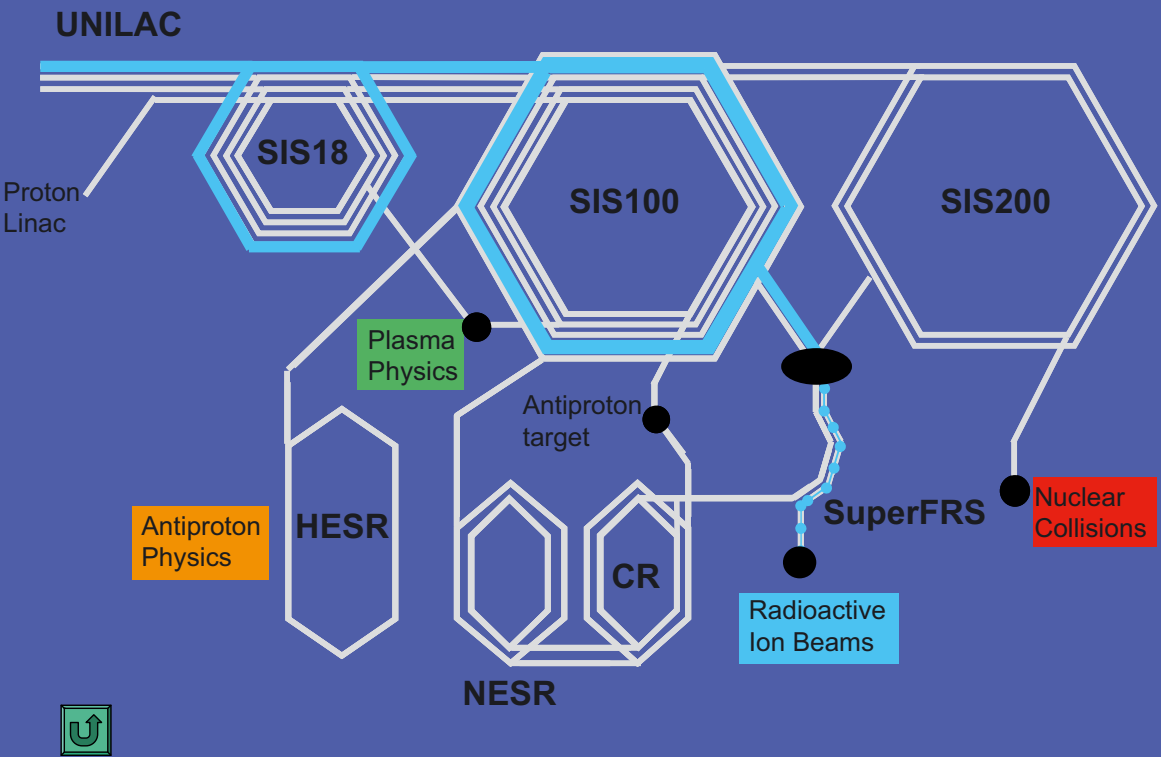




\section{Ions for Nuclear Collisions}

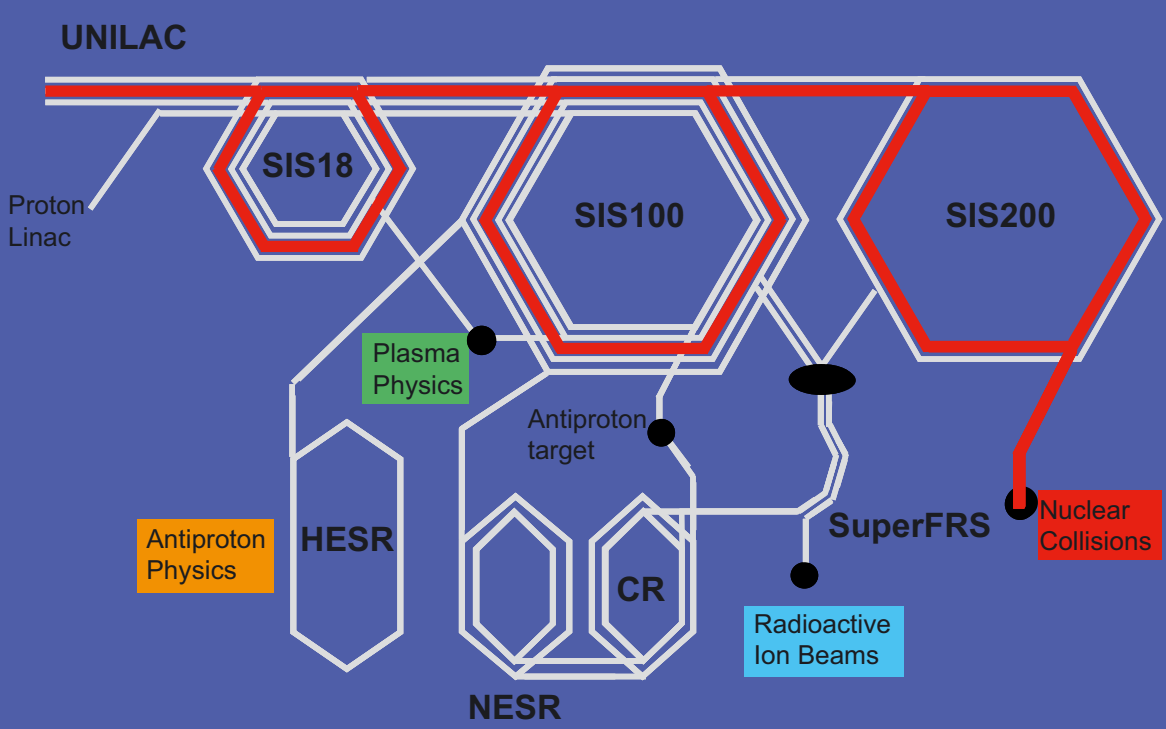

lons for Plasma Physics

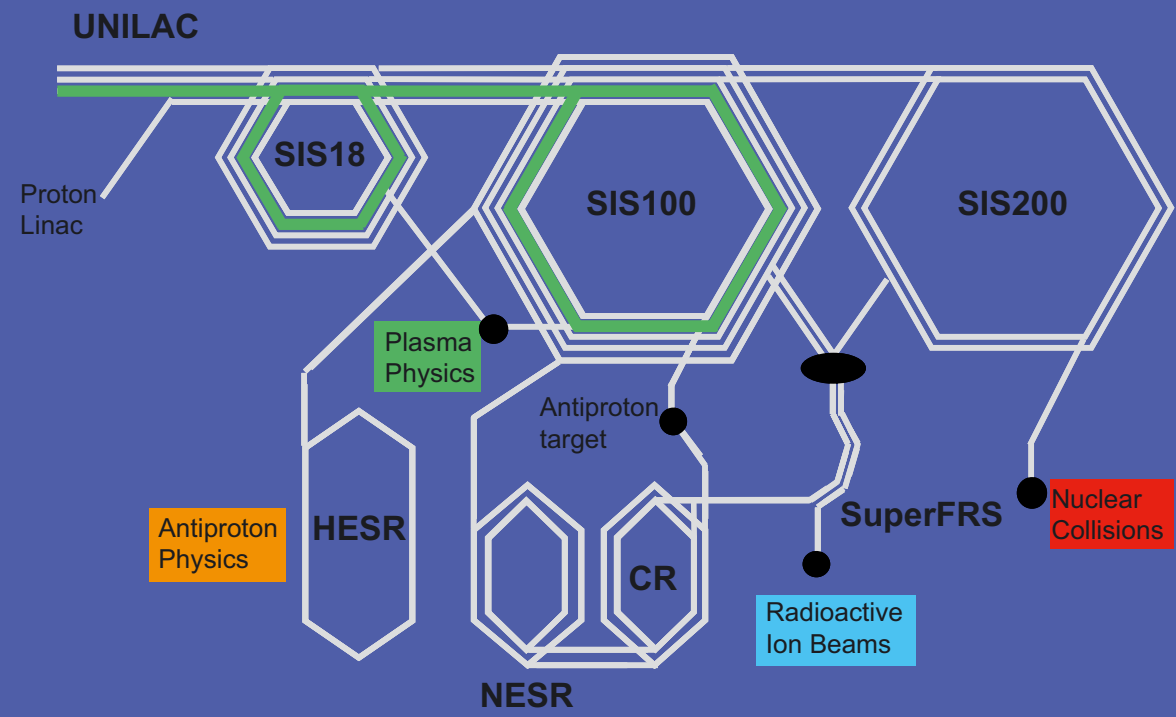




\section{Ions for Plasma Physics}

\section{UNILAC}

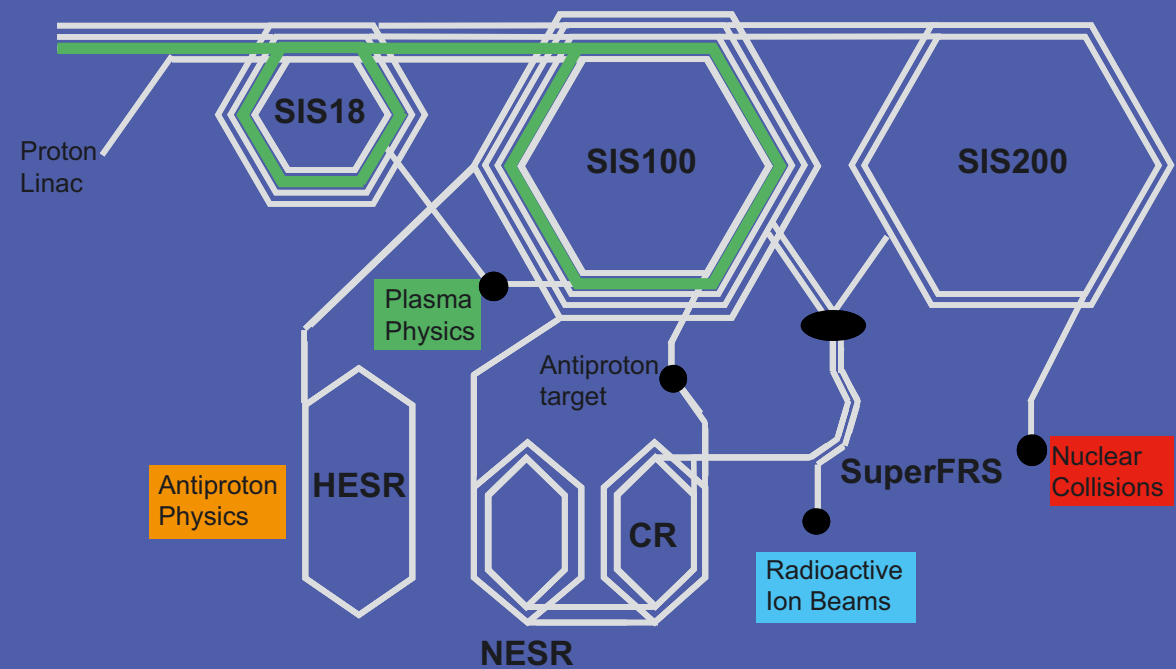

Ions for Plasma Physics

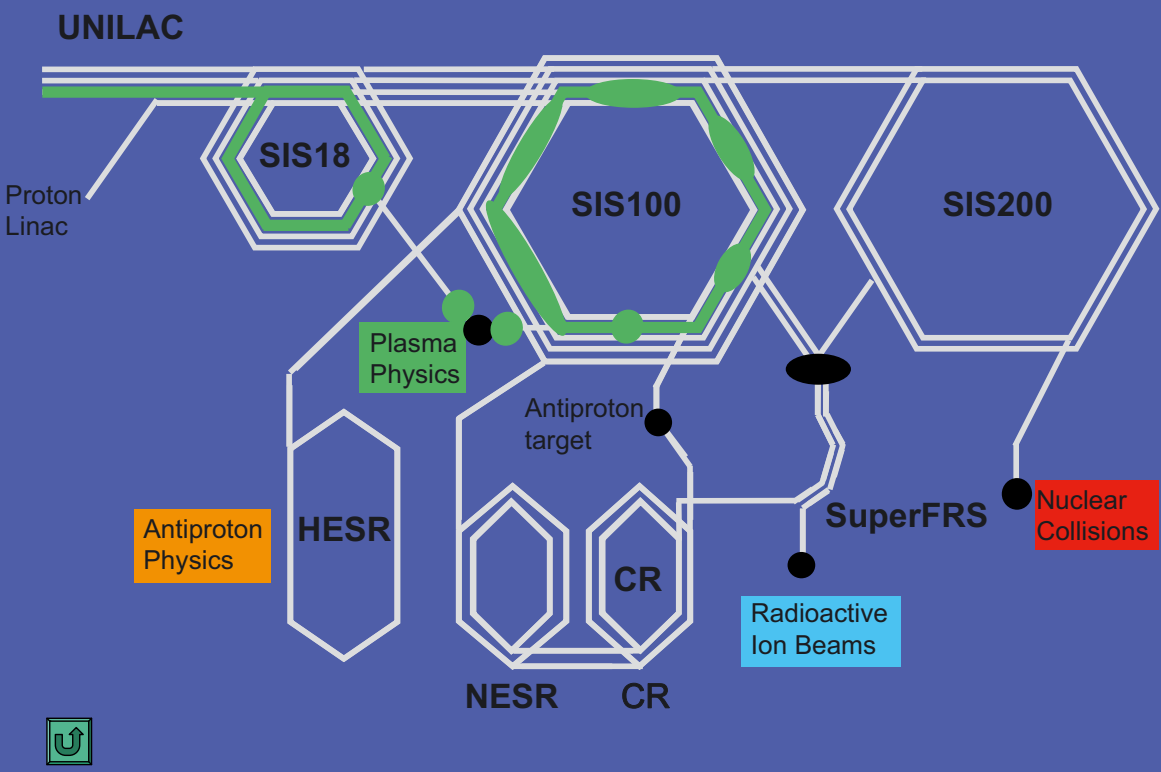


Protons and Antiprotons ..........

\section{UNILAC}

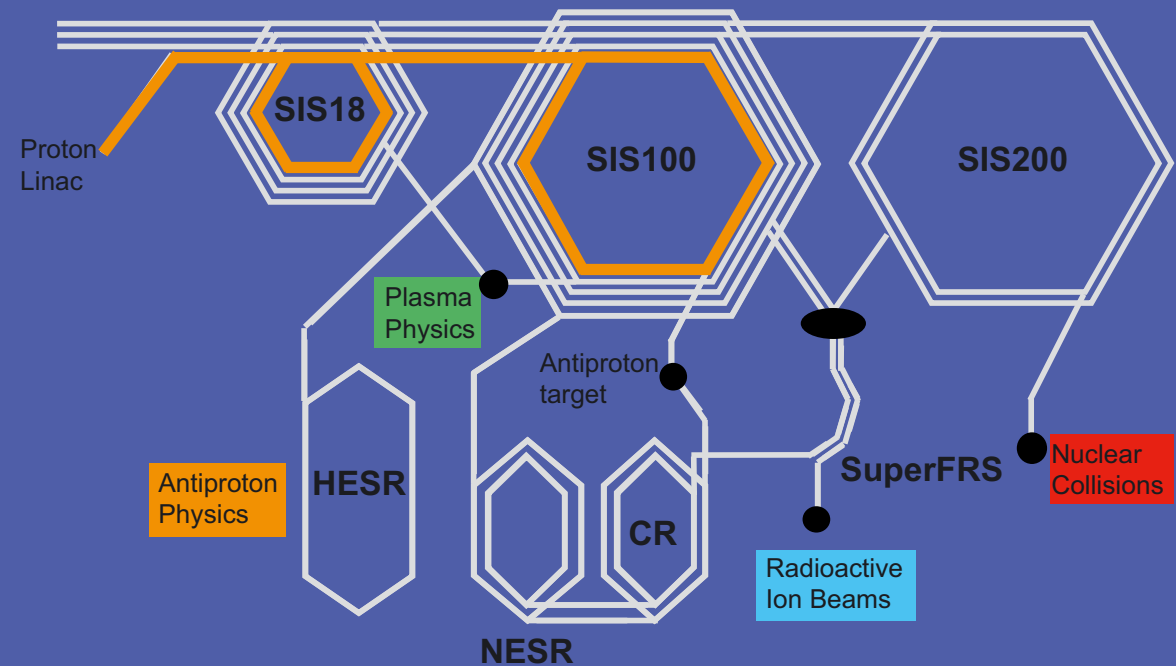

Protons and Antiprotons

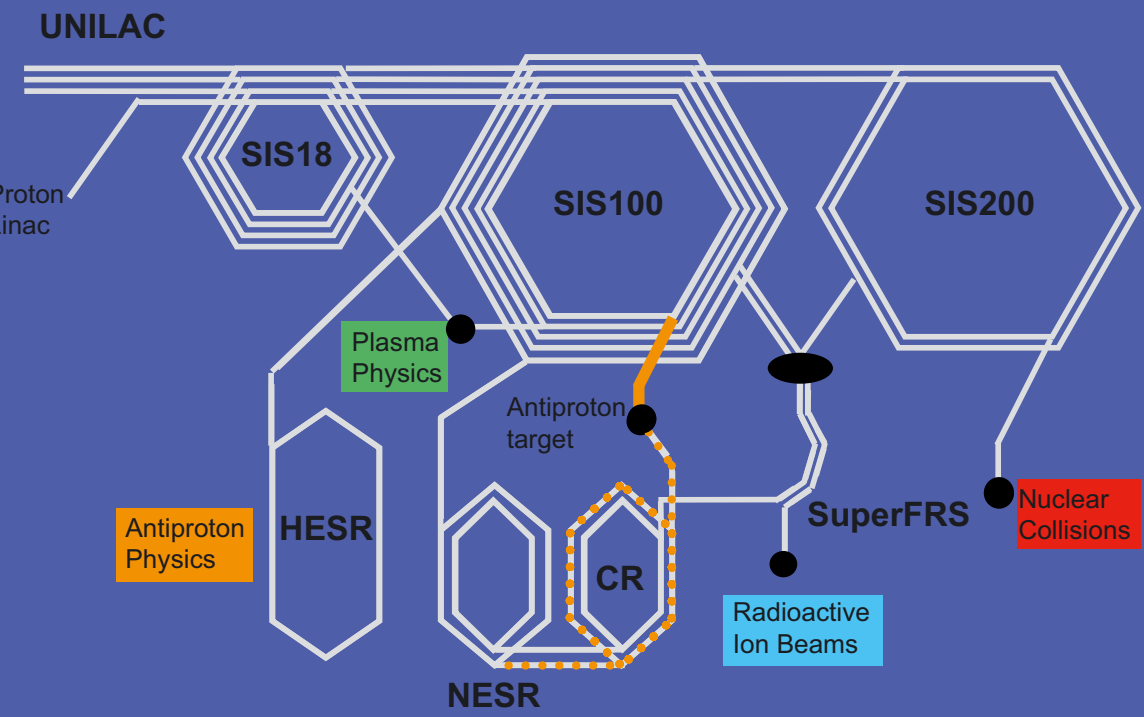




\section{Protons and Antiprotons ..........}

\section{UNILAC}

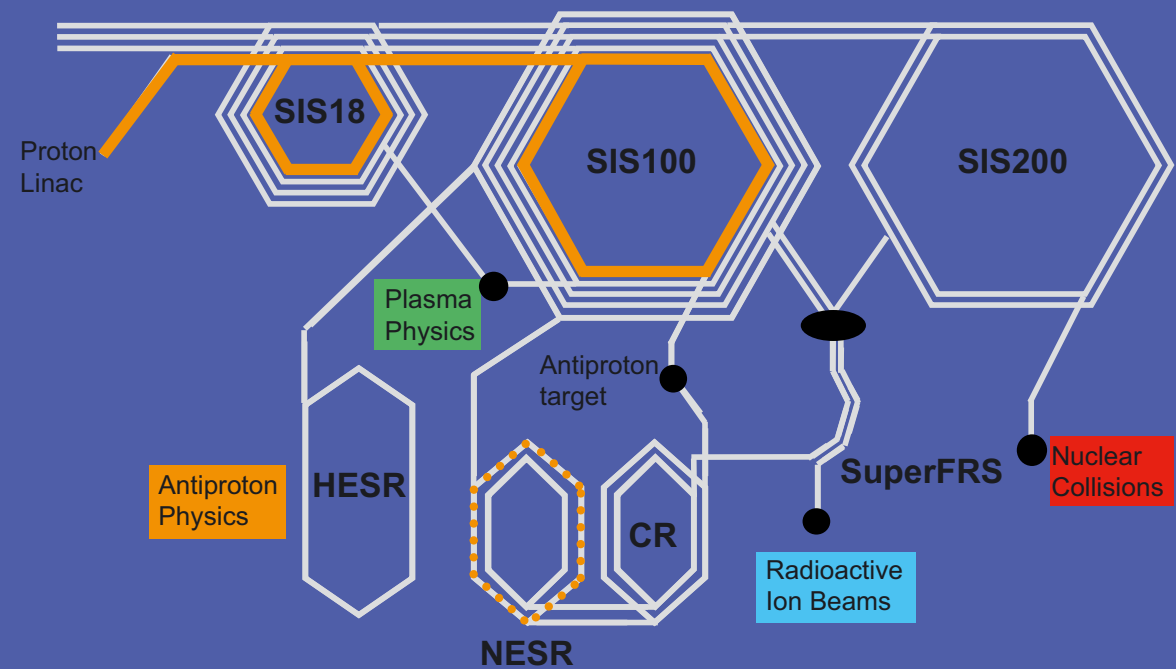

Protons and Antiprotons

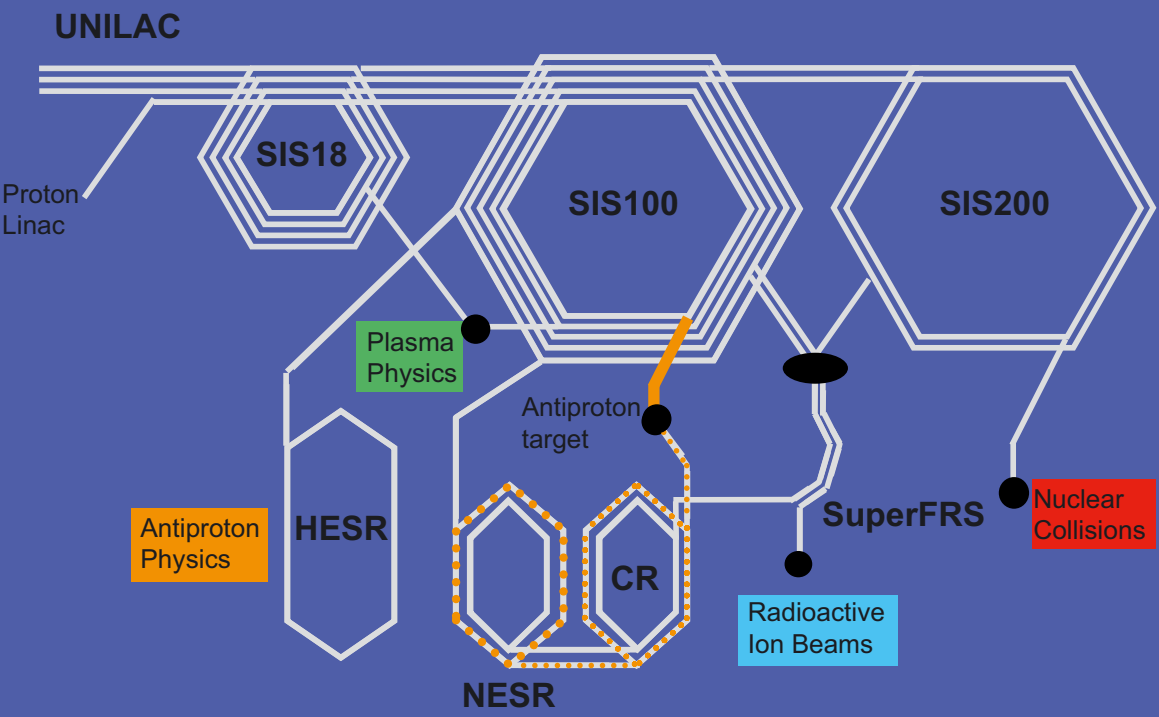


Protons and Antiprotons ..........

\section{UNILAC}

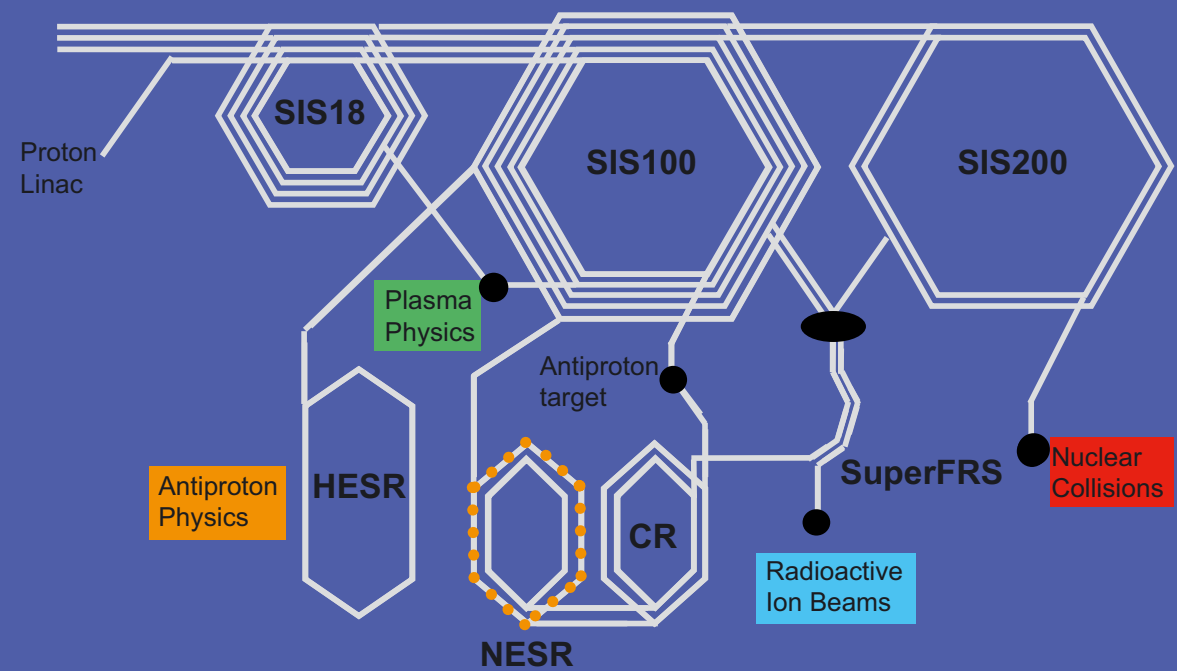

(0) D

\section{Protons and Antiprotons}

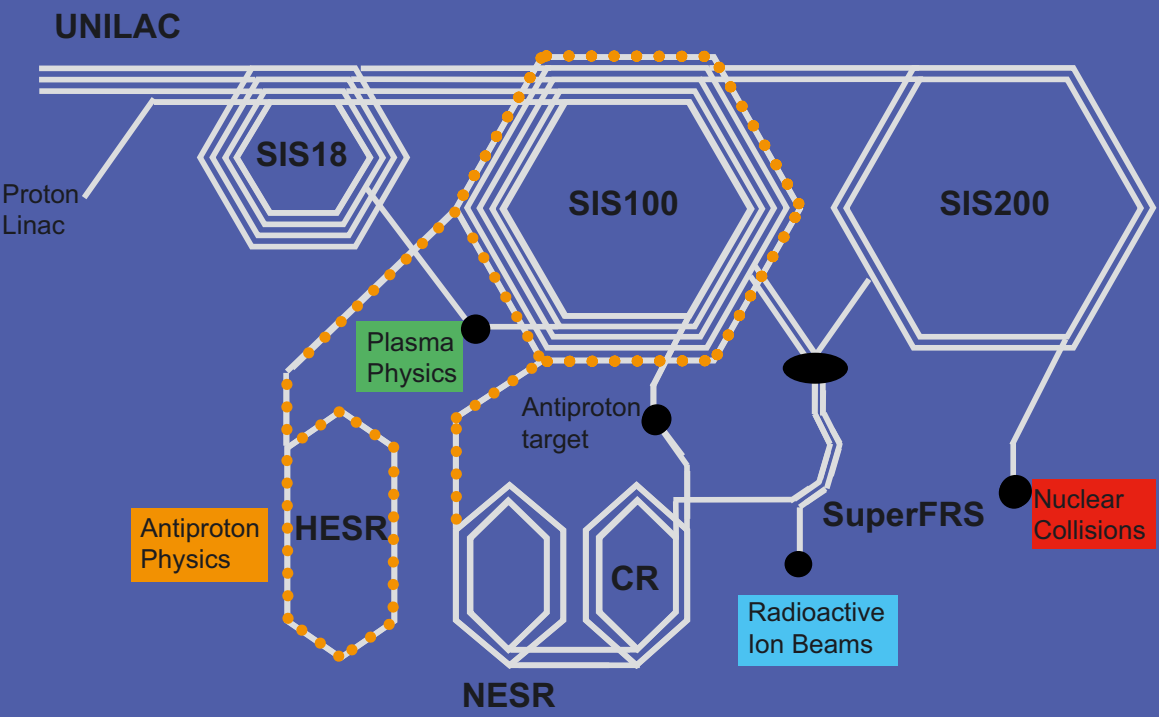




\section{Protons and Antiprotons ..........}

\section{UNILAC}

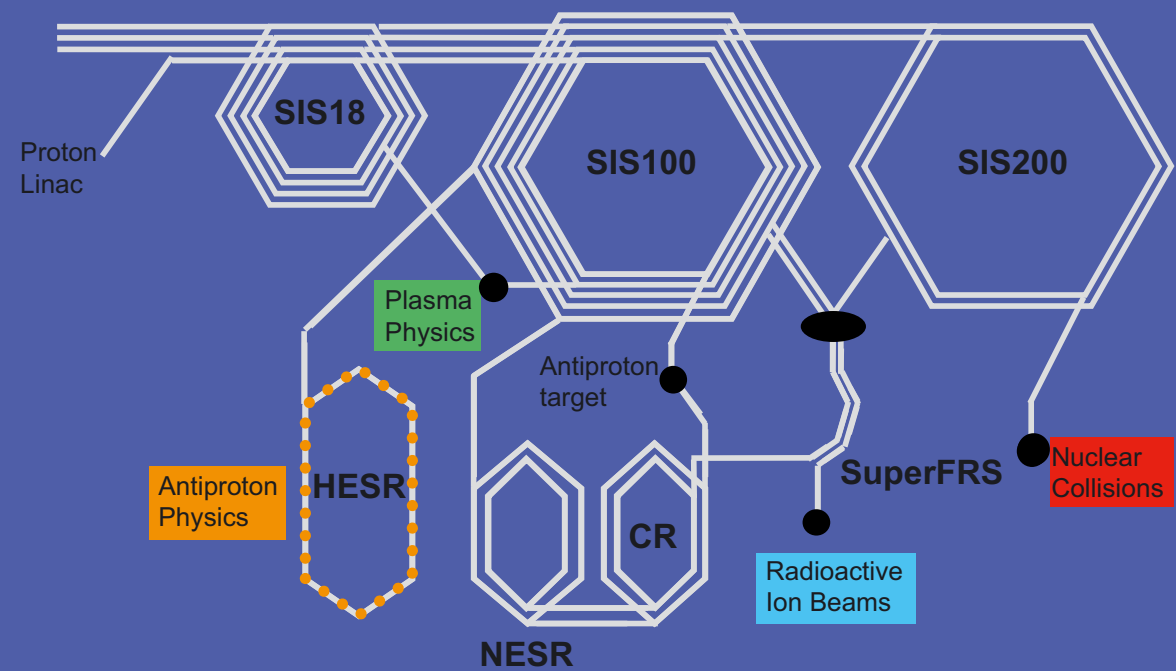

(U)

\section{Parallel Operation}

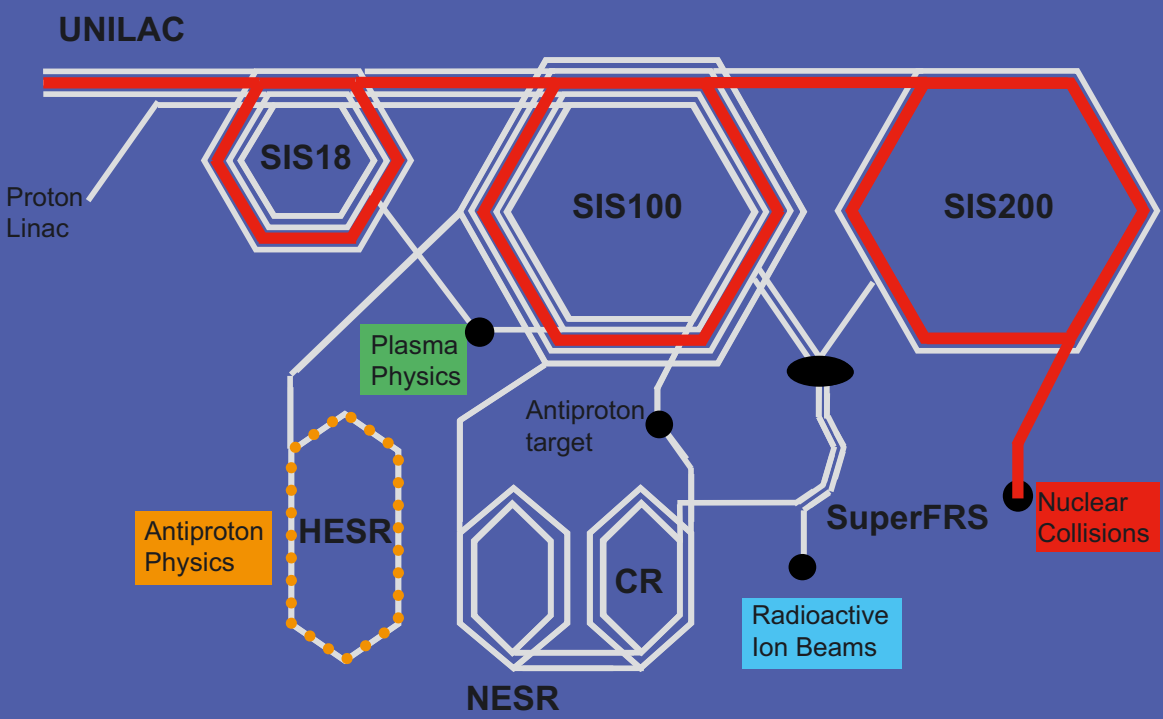




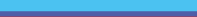
Parallel Operation

\section{UNILAC}

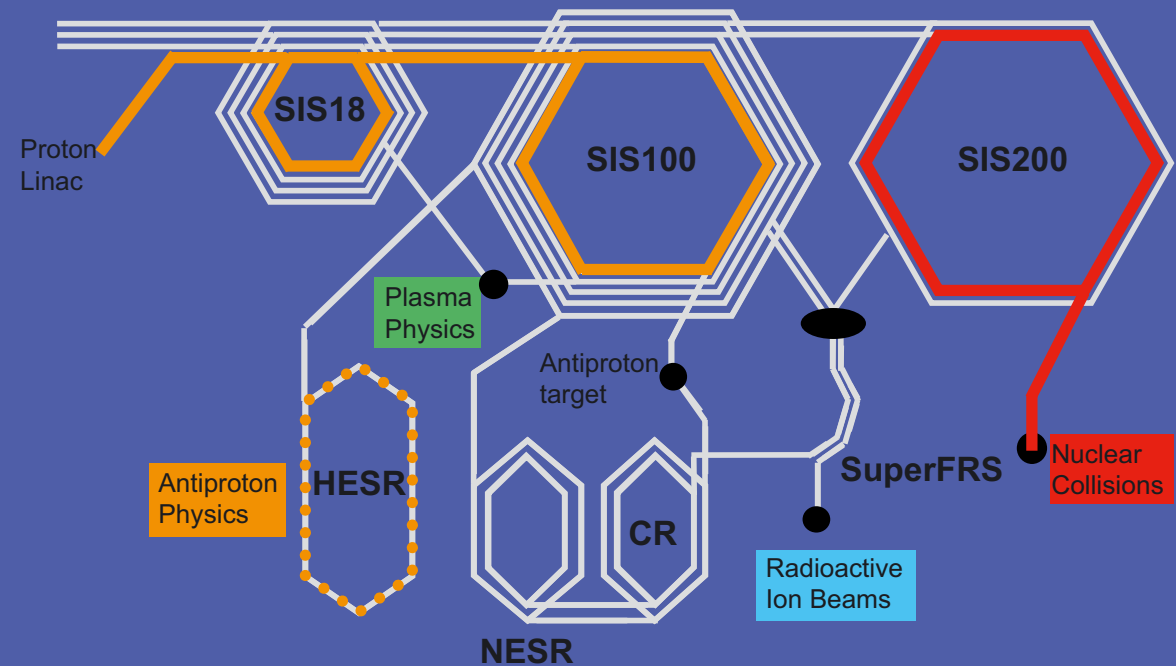

\section{Parallel Operation}

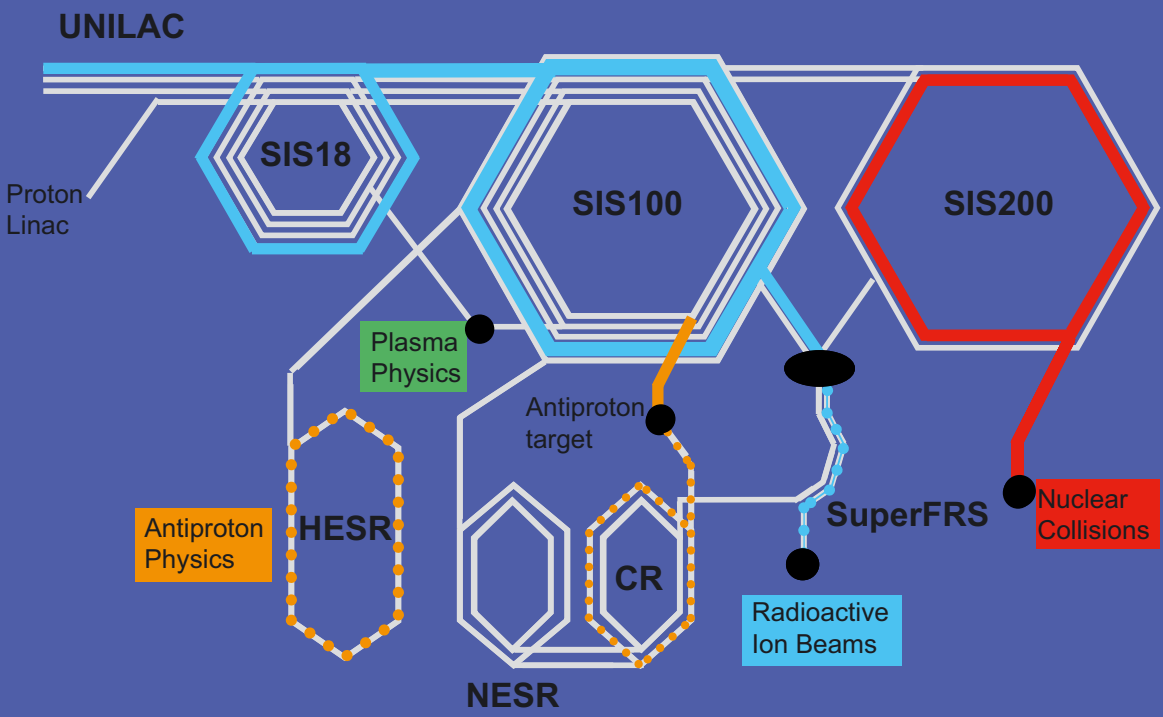



- Parallel Operation

\section{UNILAC}

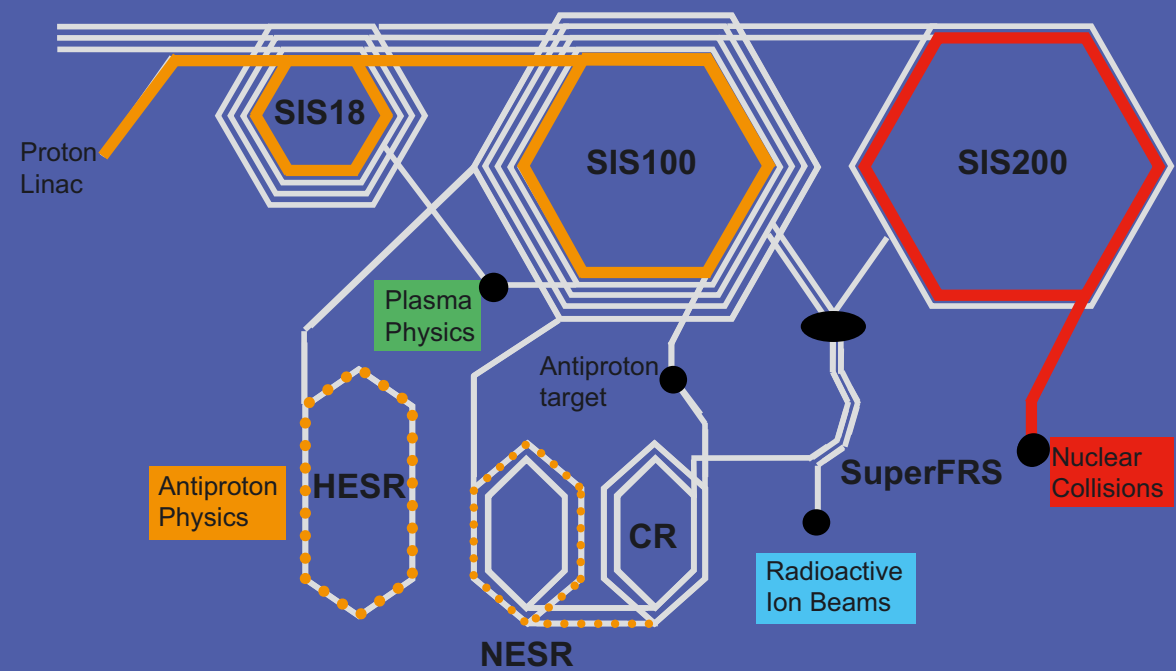

\section{Parallel Operation}

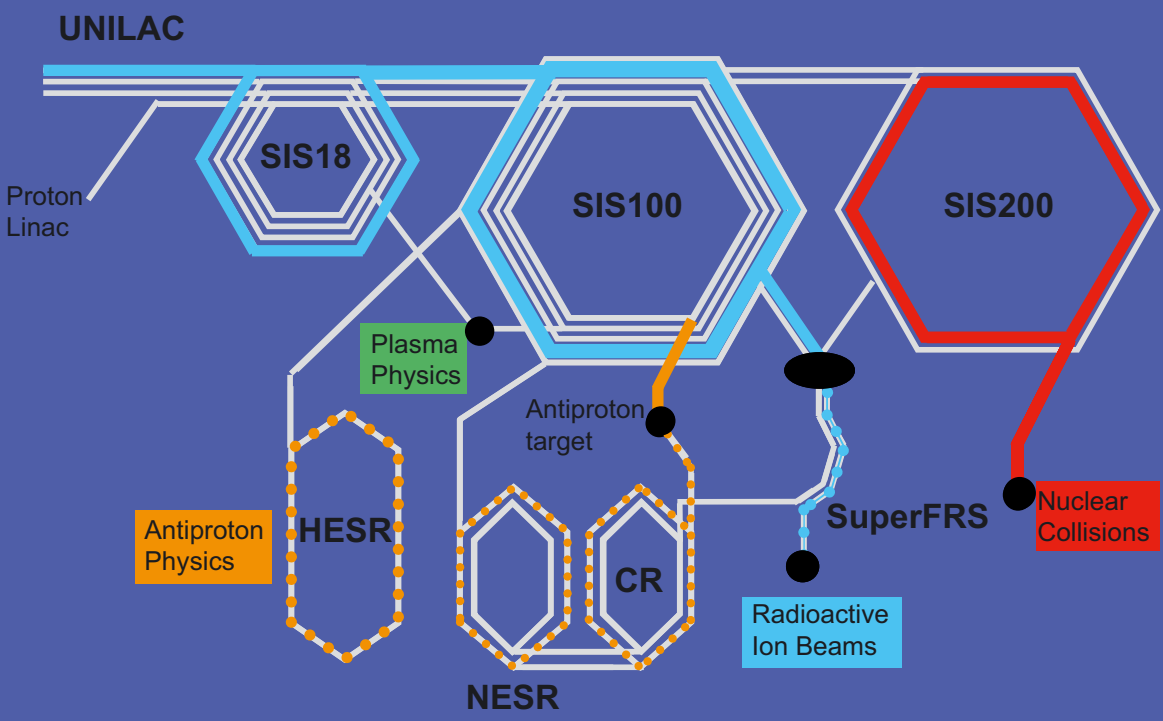




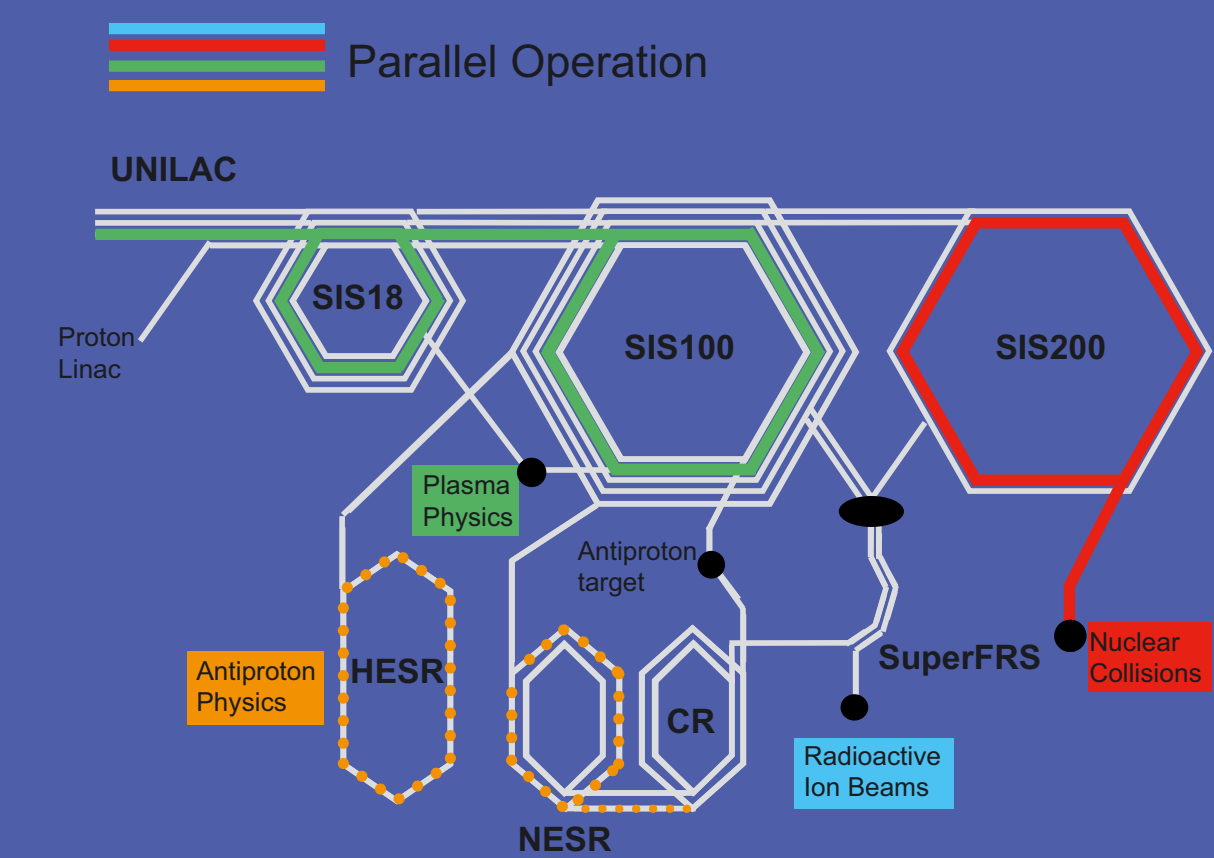

\section{Parallel Operation}

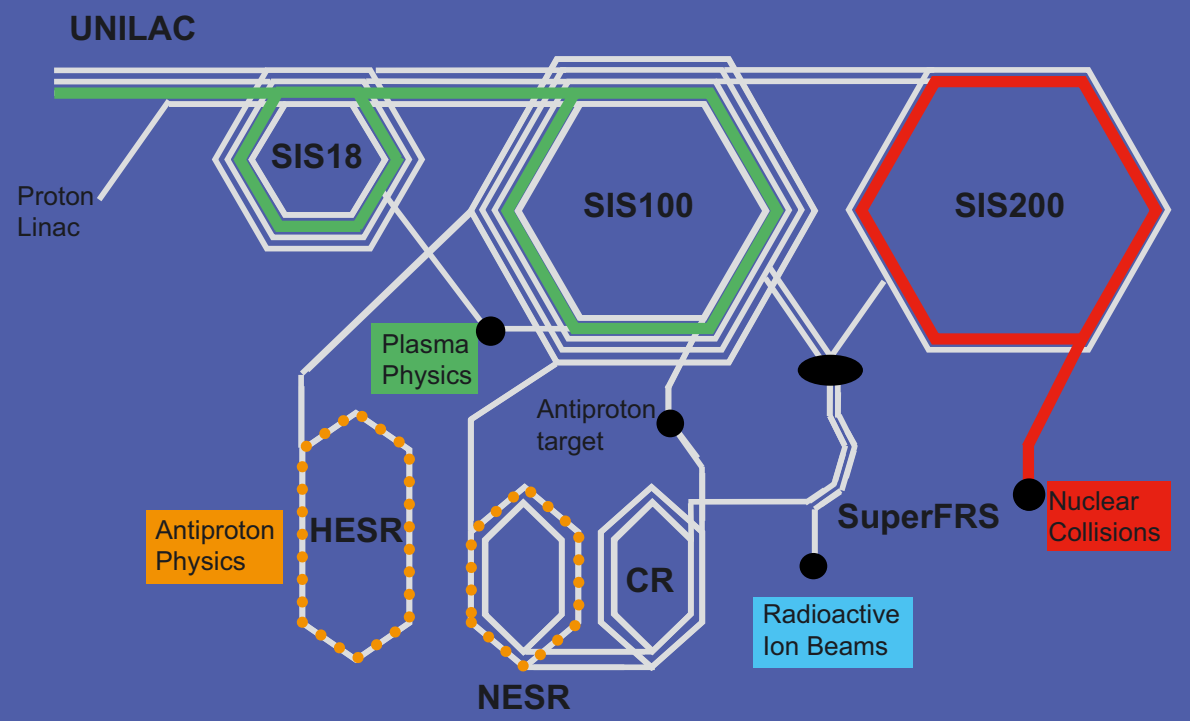




\section{Parallel Operation}

\section{UNILAC}

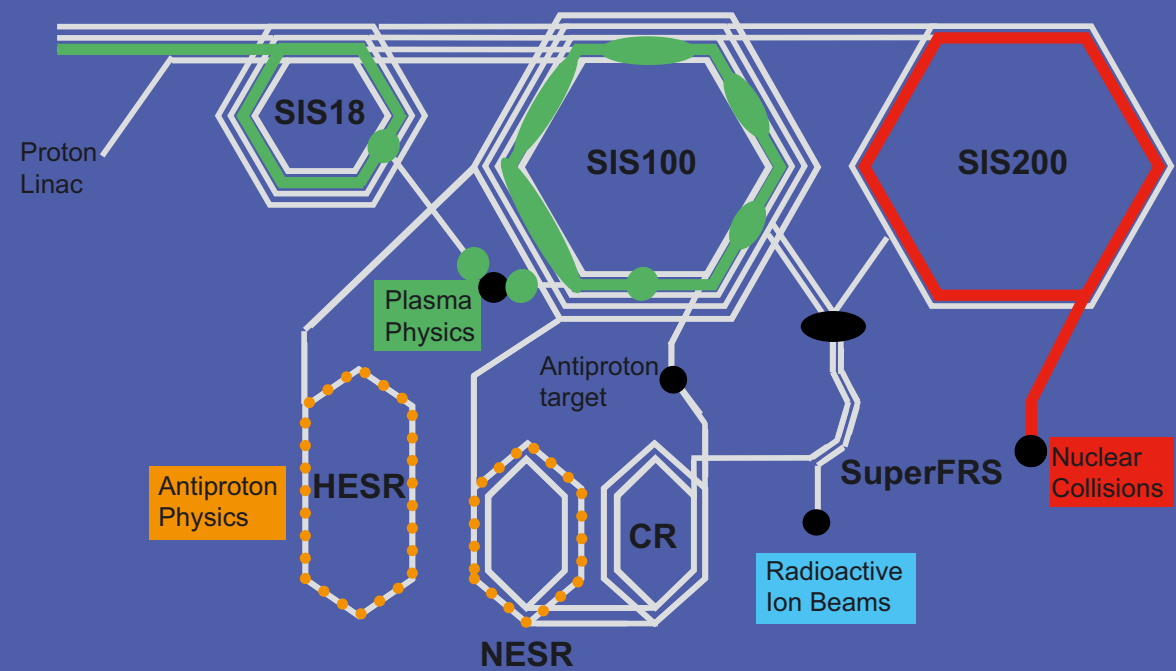

\section{Parallel Operation}

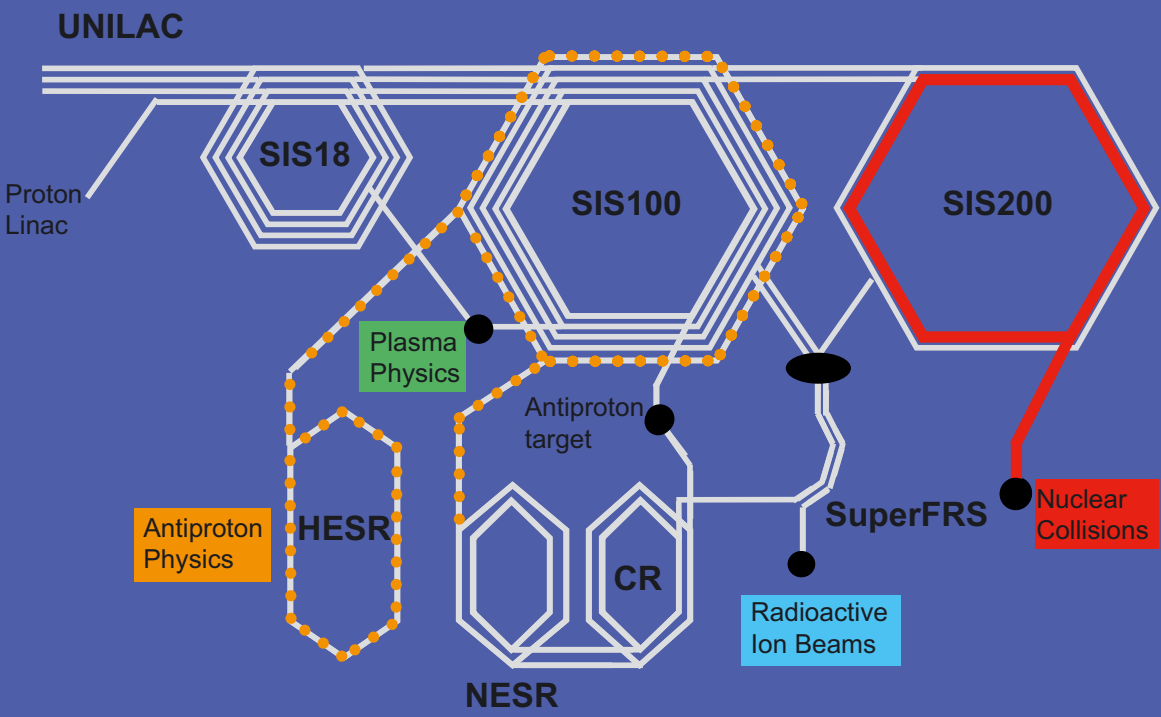




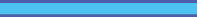
Parallel Operation

\section{UNILAC}

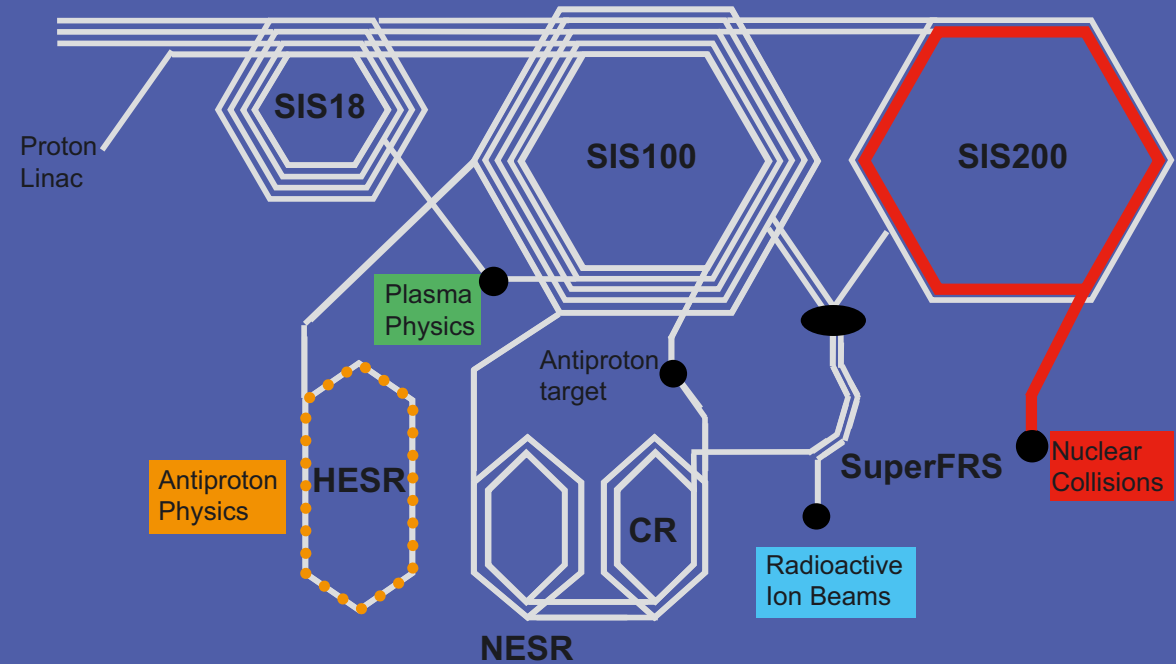

\section{Parallel Operation}

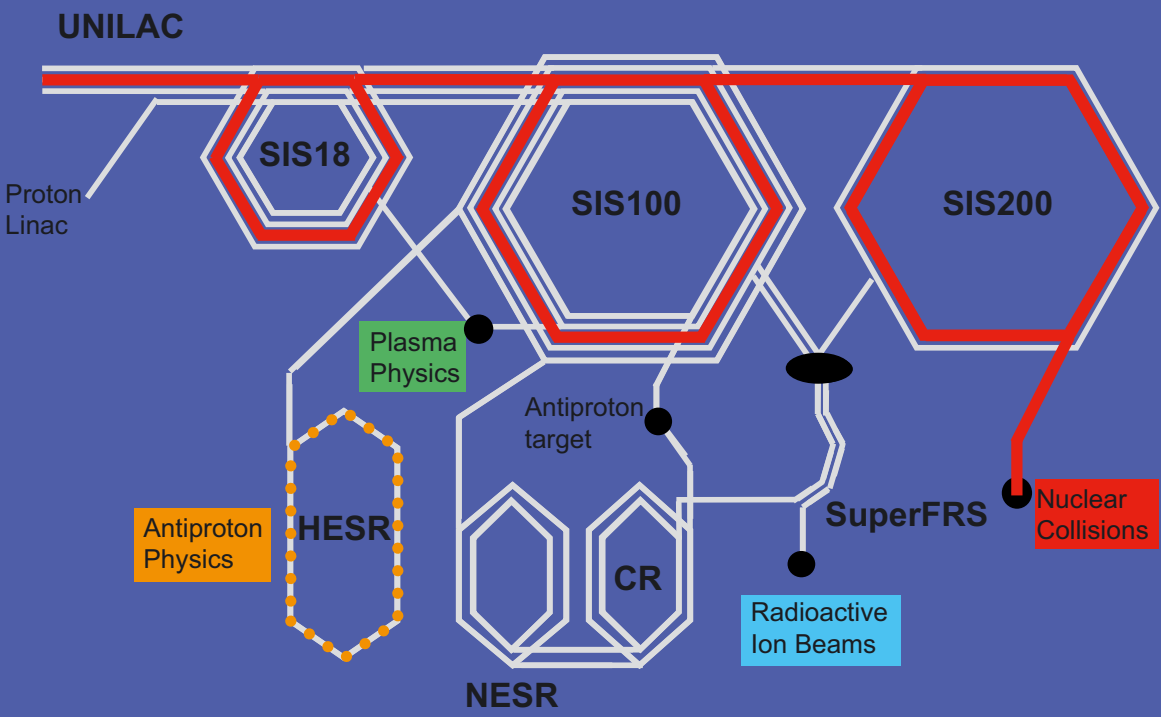


EPJ Web of Conferences

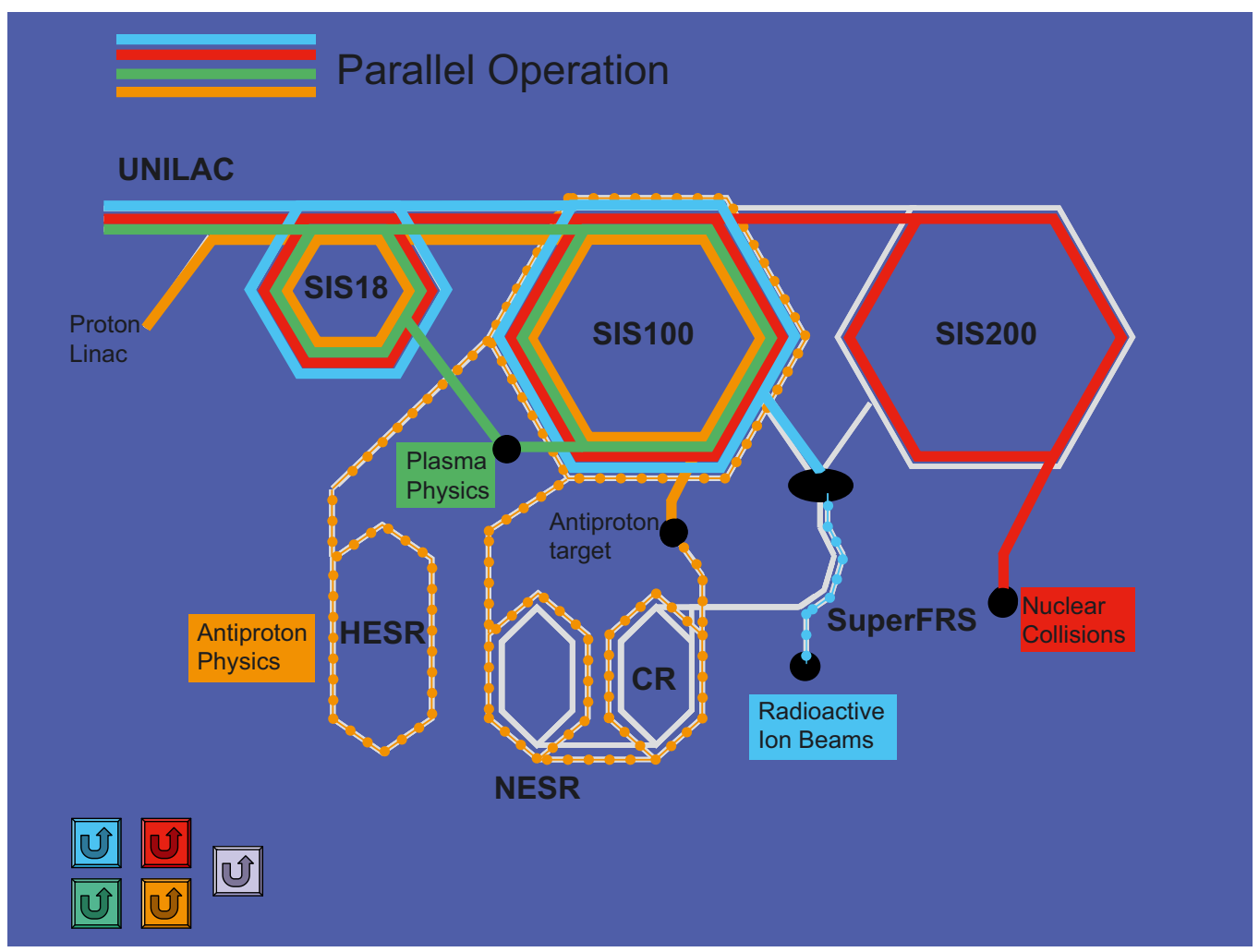

